\title{
Capsaicin is a New Gastrointestinal Mucosal Protecting Drug Candidate in Humans - Pharmaceutical Development and Production Based on Clinical Pharmacology ${ }^{1}$
}

\author{
Gyula Mózsik, Tibor Past, Tamás Habon, \\ Zsuzsanna Keszthelyi, Pál Perjési, Mónika Kuzma, \\ Barbara Sándor, János Szolcsányi, \\ M.E. Abdel-Salam Omar and Mária Szalai
}

Additional information is available at the end of the chapter

http://dx.doi.org/10.5772/58359

\section{Introduction}

Backgrounds. 1. The intact gastrointestinal mucosa is a result of excellently well regulated equilibrium between the aggressive (physical and other stress, xenobiotics, wide scale of drugs, chemicals, bacterial and viral infections) and defensive (bicarbonate secretion, mucus secretion, blood supply, prostaglandins, mucosal energy systems, etc.) factors, which are further controlled by different neural, hormonal and pharmacological mechanisms. 2 . The vagal nerve takes an essential place both in the development of gastrointestinal mucosal damage and protection. 3. The physicians have widely been applied the nonsteroidal antiinflammatory drugs (such as aspirin, diclofenac, Naproxen, etc.) as antipyretic, anti-inflammatory, painkiller and platelet aggregation inhibitor agents in healthy humans and in patients with different disorders (such as myocardial infaction, different forms of thrombophylia, rheumatoid arthritis and arthrosis or trauma) in the everyday medical practice. The administration of these drugs produces gastrointestinal complaints (mucosal damages, bleedings, perforations). So in one hand, the applications of these drugs are absolutely indicated, on the other hand, the

1 Important note: The term "capsaicin" is used in the text, however capsaicinoids of natural origin are used for during different studies.Some times the term "capsaicinoids" are used to emphasize their plant origin. 
applications of these drugs are contraindicated from the points of gastroenterologist. 4. We earlier proved clearly that the small doses of capsaicin (given orally in doses of nanogram to microgram / $\mathrm{kg}$ in animal experiments and 200 to $800 \mu \mathrm{g}$ in human observations) prevents the aspirin, indomethacin (a mixed COX-1 and COX-2 inhibitor)-induced gastrointestinal mucosal damage.

Aims: 1. The development and pharmaceutically production of the planned production of new gastrointestinal mucosal protective drug (capsaicin alone and/or capsaicin in combination with different nonsteroidal anti-inflammatory drugs) for the use in human healthy beings and in patients. 2. For the development and production of a new drug, we had to respect the following aspects: a. the knowledge of the chemical composition of natural origin capsaicin(oids); $\mathbf{b}$. problems of the analytical measurements of capsaicin(oids) in the biological samples (in animals and humans); c. correct and complete preclinical dossier; $\mathbf{d}$. correct dossier of acute and chronic toxicological studies with capsaicin (including the different tests) in two speciments of animal experiments; $\mathbf{e}$. existence of a complete drug master file (DMF); $\boldsymbol{f}$. the exclusion of pesticides, fusariums, aflatoxin and other toxicological agents (which are used during the plant cultivation in the different countries) from the capsaicin(oids) preparations used for drug production; g. to collect the necessary permissions from the different international and national authrorities before starting of pharmaceutical production of drug alone or in combinations; h. different pharmaceutical controlling measurements and other pharmaceutical aspects of planned drug or drug combinations (such as stability, drug preparation, different pharmaceutical technologies, etc.); i. preparation of different protocols for human human phase examinations (especially for human phase I.); $\mathbf{j}$. to receive the permissions from the the different national authorities to carried out the prepared protocols before starting of the classical pharmacological studies; $\mathbf{k}$. to gather the necessary numbers of experts from the very different scientific fields, who are able to solve all of above mentioned scientific, pharmaceutical, research problems (in animal experiments and in human observations); 1 . to find accredited institutes to perform human clinical pharmacological (phase) examinations. 2. When we solved all of above scientific problems, the human phase I. examinations with capsaicin alone and with combination+aspirin and capsaicin+diclofenac, the human phase I. examinations were in the Clinical Pharmacological Units of First Department of Medicine and of Institute of Cardiology, Medical and Health Centre, University of Pécs, Hungary, meanwhile the pharmacokinetic measurements were done in the Laboratories of PannonPharma Pharmaceutical Ltd., Pécsvárad, Hungary. 3. The aims of these human phase I. examinations were: a. to measure of capsaicin in the plasma of male human healthy subjects, when the capsaicin was given orally (in doses of 400 and $800 \mu \mathrm{g}$ ) alone or in combination with aspirin (500 mg orally) and with diclofenac (100 mg orally); $b$. to measure the pharmacokinetic parameters $\left(\mathrm{C}_{\max }, \mathrm{T}_{\max }\right.$

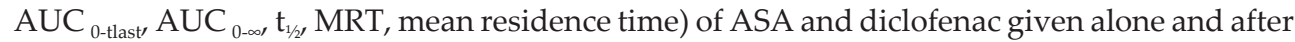
in combinations with different doses $(400$ and $800 \mu \mathrm{g})$ of capsaicin; c. to study extents of inhibitory effect on platelet aggregation of ASA and diclofenac on the epinephrine-induced platelet aggregation.

The observations were carried out on volunteers of healthy male human subjects. The volunteers were selected by special experts of human clinical pharmacology based on the strict 
criteria of inclusion and exclusion. The time period of phase I. examinations was maximally 8 weeks, from which two weeks for randomisation of the volunteers (15 in each study), the clinical pharmacological study was done in five sequences during 5 weeks, and the post-study procedure took one week after the closing the clinical pharmacological studies. The observations were carried out in random allocation, and the human phase I. examinations covered hospitalization (two days) and outpatients (4 days) periods. The observations were carried out according to the written and permitted protocols ("Human phase I. single-blind study comparing the pharmacokinetic properties of ASA after single administration alone and coadministration with two different doses of capsaicin (400 and $800 \mu \mathrm{g}$ ) and evaluating their safety in healthy male subjects“. Protocol number: 1.4.1; EudraCT number: 2008-007048-32 and "Human phase I. single-blind study comparing the pharmacokinetic properties of diclofenac after single administration alone and co-administration with two different doses of capsaicin (400 and $800 \mu \mathrm{g}$ ) and evaluating their safety in healthy male subjects". Protocol number: 1.4.2; EudraCT number: 2008-007050-36).

Our observations were to produce clinically and pharmaceutically a new capsaicin containing drug and drug combinations started from 2005 as an "innovative drug research", meanwhile the human phase I. examinations started from March 10, 2011 and finished by December 31, 2013 (including the examinations, pharmacokinetic measurements, mathematical analysis of obtained results, closing of written reports).

Main results and conclusions: 1 . The presence of capsaicin and dihydrocapsaicin (after orally given capsaicinoids of 400 and $800 \mu \mathrm{g}$ ) was not able to detect in the plasma of the healthy male volunteers, who were treated with capsaicin(oids); 2 . The capsaicin(oids) does (do) not modify the absorption, metabolism and excretion of orally given ASA and diclofenac; 3 . The capsaicin(oids) does(do) not modify the epinephrine-induced platelet aggregation by ASA and diclofenac, meanwhile the different doses of capsaicin(oids) alone has (have) no direct action on the epinephrine-induced platelet aggregation; 4. We have learned a lot of new research, juristic, patent, pharmaceutical and other clinical pharmacological problems (from basic research problems, requisted animal toxicological examinations by the different authorities,

preparation of preclinical dossier, the different pathways for obtaining official permission from the different authorities to carried out the human clinical phase examinations, chemical qualification of capsaicin(oids) in the plasma in treated animals and human healthy subjects);

5. We were able to start with the development and production of pharmaceutical production of gastroprotective capsacin(oids) containing drug alone and in combinations with ASA and diclofenac based on the internationally accepted laws of human clinical pharmacology; 6 .

We established the scientific basis of the capsaicin-sensitive afferentation vs. gastric mucosal protection against the different noxious agents (including the nonsteroidal anti-inflammatory drugs) in human healthy subjects and in patients treated with different anti-inflammatory drugs. 


\section{Historic backgrounds}

The biological regulation of living organ is very complex process, including the efferent nerves as well as afferent nerves as recently discovered. These regulatory processes influence main cellular physiological mechanisms. Of course, for the understanding these mechanisms we have to learn the principal physiological laws at the level of cells (including the classical physiology, biochemistry and in different pathological circumstances) (Mozsik, 2006).

The primary aim of pharmacology is to give different biological or chemical substances, which are able to modify cell functions under normal and pathological conditions (Mózsik et al., 1997; Mózsik, 2006). The field of pharmacology changed significantly nowdays by the application of various research trends (chemical synthesis of new drugs, drugs of plant origin, molecular biology plus immunology, etc).

We deal briefly in this paper with the interdisciplinary challenges in new drug (and drug combinations) production of plant origin capsaicin (alone or in combination with NSAIDs) modulating (used in doses, which have stimulatory actions on the capsaicin-sensitive afferent nerves) pharmacologically the afferent nerves in the gastrointestinal (GI) tract. From that point of views we carefully and critically evaluated the correlations between the plant origin capsaicinoids, NSAIDs with GI mucosal damage and preventions in animals and human healthy beings (Abdel Salam et al., 1994; 1995a,b,c,e,f,g; 1996a,b; 1997a,b,c,d; 1999; 2001; 2006 ; Mózsik et al., 1999; 2006 a,b; 2005; 2007).

\section{Main problems of pharmacological treatment in patient with different diseases}

The patients with myocardial infarction, thromboembolic episodes, stroke of central nervous system, cancers and persons who have to be treat as preventions of different diseases (reinfarction after myocardial infarction, prevention of thromboembolic episodes produced by atrial fibrillation, cancers, after different surgical intervention and immobilization) and in healthy subjects treated with NSAIDs in order to prevent the development of colorectal cancers. The number of these groups of patients reaches to 50-60 per cent of total population in Hungary.

The majority of patients who underwent cardiac and other surgeries are treated permanently with aspirin (in dose of $100 \mathrm{mg} /$ day/person). This is a basic stand-point of the different consensus meetings of Europe and of the World (Megettigan et al., 2006; Patrono et al., 2004; Todd and Clisson, 1990).

The administration of aspirin in absolutely indicated from medical points of view in patients mentioned above, accepting the opinion of cardiologists; however, we have to emphasize that the aspirin very frequently produces GI bleeding (which do not favour gastroenterologists). Really, there is a great and principal question from the general medical practice (and of research), namely whether all patient can be taken as the same, in whom the medical science 
should offer medical treatment from the point of cardiology, which produces severe GI disorders (bleedings, peptic ulcer). Consequently there is a contradictory medical (and evidence-based proved) standpoint between the cardiologists vs. gastroenterologists (during the treatment of one patient, and as well as in the treatment of populations of patients mentioned above).

Another big population of patients suffers from different degenerative joint diseases, trauma or from acute and chronic pain. These patients have to receive permanent treatment with NSAIDs. The NSAIDs are not GI protective agents neither in healthy person and nor in patients with these disorders. Large portion of patients appearing on gastrointestinal wards suffer from the drug-induced side effects.

Patients with NSAIDs-induced gastrointestinal disorders (blood losing, bleeding, peptic ulcer) represent a significant number of populations. Furthermore, these patients have to treat permanently by different NSAIDs.

The actions of NSAIDs are associated with the selective and non-selective inhibitory properties on cyclooxygenase system (emphasizing the key role of COX-1 and COX-2). Aspirin is a specific COX-1 inhibitor; meanwhile other NSAIDs applied in the clinical practice represent the compounds acting as non-selective COX-1 and COX-2 inhibitors. Recently, the specifically acting compounds, inhibiting COX-2 enzyme, have been produced to reduce gastric mucosal damage, however, the number of myocardial infarction was increase in such patients (Megettigan et al., 2006; Patrono et al., 2004; Tood and Clissold, 1990; Couzin 2004a,b; Lenzer, 2004).

We have to emphasize clearly, that the small doses of capsaicin are able to prevent gastric mucosal bleeding induced by NSAIDs (both COX-1, COX-2 inhibitors). It is true that COX-2 inhibitors produce smaller side effects in GI tract of patients; however these compounds have no inhibitory properties on the thrombocyte aggregation. When the patient with angina pectoris and degenerative joint disease received only COX 2 inhibitors, than the number of patients with myocardial infarctions and of cardiac origin death increased significantly (“Vioxx story") (Couzin, 2004a,b ; Lenzer, 2004; Green, 2005; Lawler, 2005; Tanne, 2006a,b,c). Recently, the European and American Consensus Meetings uniformly accepted the salicylate application in about dose of $100 \mathrm{mg} /$ day and of $300 \mathrm{mg}$ /day in cumarine resistant patient (Megettigan et al., 2006; Patrono et al., 2004).

Our aim was to product a capsaicin containing drug or drug combinations with a NSAID for the treatment of above mentioned groups of patients hoping that application of these compounds will be able to prevent the NSAIDs induced GI side effects in patients (Mózsik et al., 2009a,b, 2010; Szabo et al., 2013).

\section{Experts' opinion up to 2008}

We successfully applied funding for innovative academic pharmacological and pharmaceutical industrial research to the National Office for Research and Technology (Hungary) in 2005 (Regional University Science Centre Pécs, Hungary, MEDIPOLISZ, Pázmány Péter Pro- 
gramme RET-II, 08/2005) for time period of 2006-2008, and later on BAROS GÁBOR Programme, Hungary (REG_DKI_O,CAPSATAB) for time period of 2010-2011.

The aims of these research programmes (in these time periods) were to produce new capsaicin(oids) drug alone and in different combinations for patients with different gastrointestinal disorders, for patients with myocardial infarctions (orally applicable combinations of capsaicin(oids) with aspirin) and for those with chronic degenerative locomotive diseases (orally applicable capsaicin(oids) with diclofenac and Naproxen).

Twenty-one researchers (chemist, pharmaceutical chemist, physicians, clinical pharmacologists, pharmaceutics, laboratory experts, biologists, engineers and agricultural engineers) have participated in this innovative pharmacological and pharmaceutical research.

The capsaicin(oids) are well-known species, which are able to modify the action of capsacinsensitive afferent nerves. Their actions are dose-depependent, and they are capable to modify the neurogenic inflammantion, pain, and the defence of the various target organs against different noxious agents.

The research of capsaicin is a traditional and internationally well accepted research line at Pécs University, Hungary (Department of Pharmacology and Pharmacotherapy, First Department of Medicine in a well successful cooperation) in the 50 years period. We arrived to the production of orally given capsaicin(oids) new drug and capsaicin(oids) plus nonsteroidal anti-inflammatory drugs in the time of our innovative pharmacological and pharmaceutical research in the 2005.

The production of new drug or different drug combinations represented an interdisciplinary challenge for all of us (which significantly differed from the traditional basic and clinical research).

The first step of our common work was to write an interdisciplinary experts' opinion based on the most important research data from the capsaicin research to be used for the new drug production, for receiving different permissions from the different authoritities to start with the classical clinical pharmacological studies.

The experts's opinion was prepared (Mózsik et al., 2007b), and this material was published in Inflammopharmacology (Mózsik et al., 17:113-150, 2009a). This part of book chapter deals with these experts' opinion (subchapter of 3). The authors summarized their obtained significantly different results in these studies from the years of 2005 to 2008 (Mózsik et al., 2009b).

\subsection{Brief introduction}

Capsaicin is an active ingredient of red pepper and paprika. These plants have been well known in about 9500 years, and these have been applying in the every day of the culinary practice.

It was an important discovery that the capsaicin (capsaicin, dihydrocapsaicin, nordihydrocapsaicin and other capsaicinoids) specifically modify the function of capsaicin sensitive afferent nerves (Jancsó et al., 1967; 1968; 1970). 
The action of capsaicin on the capsaicin sensitive afferent nerves is dose dependent (Szolcsányi and Barthó, 1981; Szolcsányi, 1984, 1997, 2004; Abdel-Salam et al., 1999; 2001; Mózsik et al., 2001). Szolcsányi indicated four different stages of capsaicin action (depending on the dose and duration of the exposure of the compound): a. excitation (stage 1); b. sensory blocking effect (stage 2); c. long-term selective neurotoxin impairment (stage 3) and d. irreversible cell destruction (stage 4) (Szolcsányi, 1984). The stages 1 and 2 are reversible; meanwhile the stages 3 and 4 are irreversible compound-induced actions on the capsaicin sensitive afferent nerve. These stages of capsaicin actions can be detected in the gastrointestinal tract (Mózsik et al., 2001).

Capsaicin activates the capsaicin (vanilloid) receptor expressed by a subgroup of primary afferent nociceptive neurons (Szolcsányi, 2004). The capsaicin receptor had been cloned (Caterina et al., 1997) and turned out to be a cation channel. It is gated besides capsaicin and other capsaicinoids (some vanilloids) by low $\mathrm{pH}$, noxius heat and various pain-producing endogenous and exogenous chemicals. Thus, these sensory nerve endings equipped with these ion channels are prone to be stimulated in gastric mucosa.

The vagal nerve has a key role in the development of gastrointestinal mucosal damage and prevention (Mózsik et al., 1982). The key role of vagal nerve has been emphasized dominantly in the aggressive processes to gastrointestinal mucosa (such as peptic ulcer disease, gastric mucosal damage, etc.) both in the GI research of animal models and as well as in human clinical practice. The "chemical" and "surgical" vagotomy widely used in the treatment of patients with peptic ulcer disease in the years up to middle of 1970s (Karádi, Mózsik, 2000). By the other words, the primary aims of this therapy were to decrease the activity of vagal nerve at the level of efferent vagal nerve.

The application of capsaicin in the animals experiments was used as a specific tool to approach to the group of primary afferent nociceptive neurones (Szolcsányi, 2004; Buck and Burks, 1986; Holzer, 1998; 1991; Szállasi and Blumberg, 1999) involved in the different physiological and pathological processes.

Szolcsányi and Barthó (1981) were the firsts, who clearly indicated the beneficial and harmful effect of capsaicin in the peptic ulcer disease in rats, on dependence of applied doses of capsaicin. Later on, Holzer started with a very extensive research work with capsaicin in the field of Gastroenterology (Holzer, 1998; 1999; Buck and Burks, 1986; Szállasi and Blumberg, 1999). We also participated in the GI capsaicin research in animals experiments from 1980 (Mózsik et al., 1997). Recently, the new drug, Lafutidine, was processed in the medical treatment of GI mucosal damage (Ajioka et al., 2000; 2002; Onodera et al., 1999; 2002; Takeuchi, 2006). The Lafutidine is a $\mathrm{H}_{2} \mathrm{R}$ blocking compound together with typical capsaicin actions at the target organ.

The new and interesting results obtained with capsaicin application in animal experiments offered an excellent tool to approach the different events of human GI physiology, pathology and pharmacology. Our clinical studies with capsaicin have been started from 1997 (Mózsik et al., 1999; Debreceni et al., 1999; Mózsik et al., 2004a; 2004b; 2005). 
The aim of this paper is given a short summary on the possibility of capsaicin application as a new tool to understand the various steps of human physiology, pathology and pharmacology.

\subsection{Physiological and pharmacological research tool by capsaicin}

\subsubsection{The chemistry of capsaicinoids}

\subsubsection{Chemical composition of Natural Capsaicin}

The degree of pungency (heat or bite) of the Capsicum fruits is determined by the amount of compounds called capsaicinoids. These substances are responsible to produce the characteristic sensations associated with injestion of spicy cuisine as well as responsible for causing severe irritation, inflammation, erythema, and transient hypo-and hyperalgesia at sites exposed to capsaicinoids.

All capsaicinoids posses a 3-hydroxy-4-methoxy-benzylamide (vanilloid) pharmacophore, but differ in their hydrophobic alkyl side chain. Differences in the side chain moiety include saturation of the carbon carbon double bond, deletion of a methyl group and changes in the lenght of the hydrocarbon chain (Figure 1). Previous structure-activity studies using models for the study of acut pain and altered pain sensitivity in mice have demonstrated a strict structural requiremenet for both the vanilloid pharmacophore and a hydrophobic alkyl chain that may be saturted or unsaturated, branched or unbranched, and consisting of 8 to 12 carbon atoms for optimal binding and activation of the capsaicin receptor, TRPV1 (Bevan and Szolcsányi, 1990; Walpole et al., 1993a,b,c).

It is worth mentioning that there are some contradictions in meaning of the term "capsai-

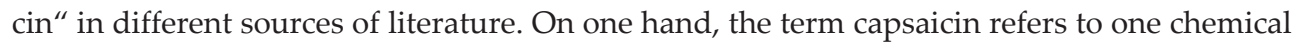
entity: (E)-8-Methyl-N-vanillyl-6-nonenamide (CAS-number: 404-86-4). On the other hand, the term capsaicin is frequently used for Capsicum extracts containing capsaicin and related capsaicinoids (e.g., see Capsaicin USP 29). These latter preparations are frequently referred to as Natural Capsaicin. In addition to the natural capsaicin preparations, capsaicin can be obtained by synthesis as well. The synthesized capsaicin is frequently referred to as trans-capsaicin, since during the syntheses not only the natural trans but the cis isomer can also be obtained.

The two main components of Natural Capsaicin (a mixture of capsaicinoids of Capsicum origin) are capsaicin and dihydrocapsaicin. There are, however, other structurally related alkyl vanillylamides (capsaicinoids) generally found in smaller amounts in Capsicum extracts. The names and structures of the main components of Natural Capsaicin are shown below (Figure 1).

Separation and quantitation of the components can be performed by gas chromatography (GC) or liquid chromatography (HPLC). The GC and HPLC chromatograms of Natural Capsaicin (Capsaicin USP 29) are shown on Figures 2 and 3, respectively. 
<smiles>COc1cc(CNC(=O)CCCC/C=C/C(C)C)ccc1O</smiles>

Dihydrocapsaicin<smiles>COc1cc(CNC(=O)CCCCCCC(C)C)ccc1O</smiles>

Nordihydrocapsaicin<smiles>COc1cc(CNC(=O)CCCCCC(C)C)ccc1O</smiles>

Homocapsaicin<smiles>COc1cc(CNC(=O)CCCCC/C=C/C(C)C)ccc1O</smiles>

Homodihydrocapsaicin<smiles>COc1cc(CNC(=O)CCCCCCCC(C)C)ccc1O</smiles>

Figure 1. Names and chemical structures of the main components of the Natural Capsaicin preparations 


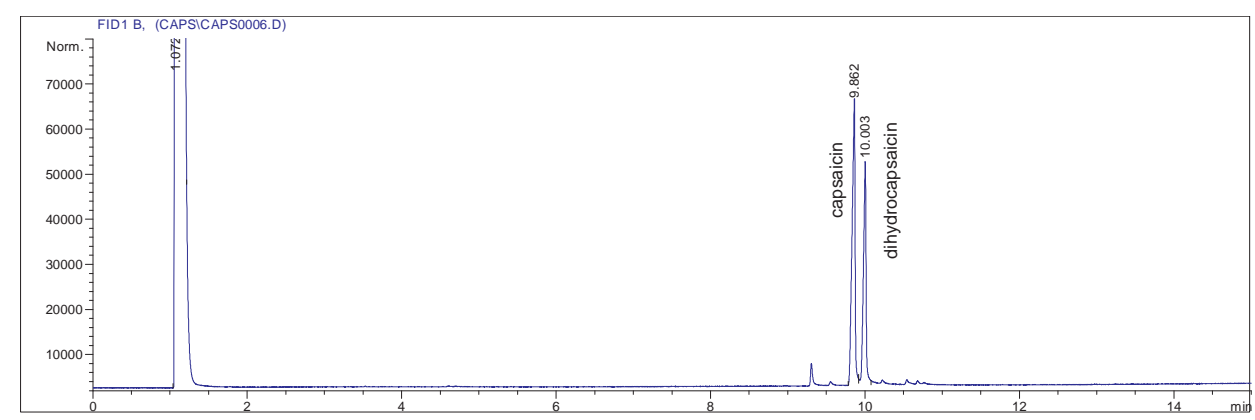

Figure 2. Gas-Chromatographic (GC-FID) analysis of capsaicin and dihydrocapsaicin of a Natural Capsaicin sample. (Identification of the components was performed by GC-MS method.) (Kuzma M. et al., unpublished results)

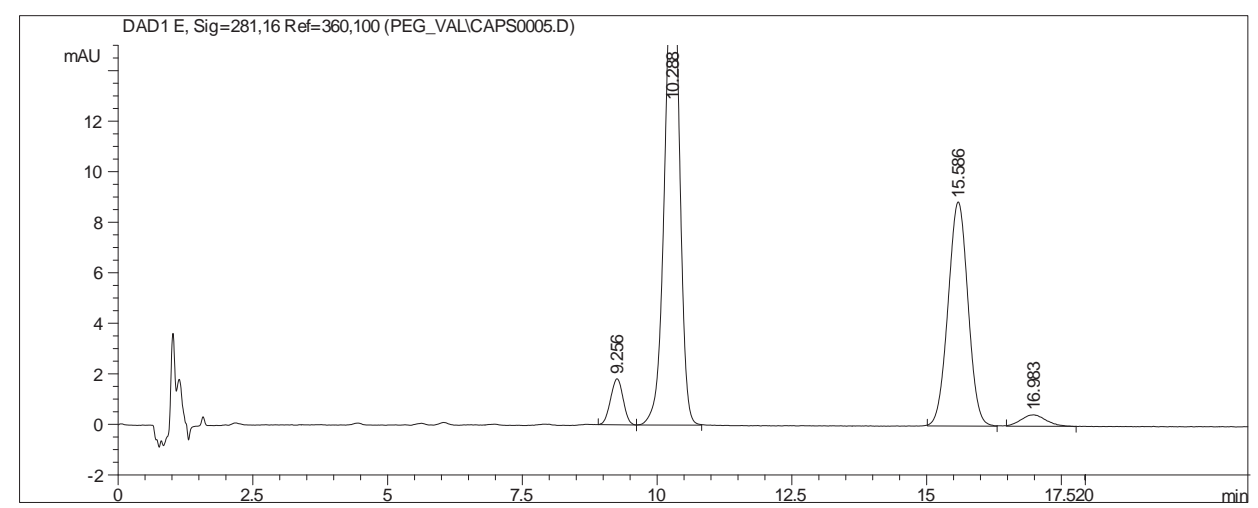

Figure 3. High Pressure Liquid Chromatography (HPLC-DAD) chromatogram of a Natural Capsaicin sample. (Retention times: Capsaicin $t_{R=} 10.29$ min, Dihydrocapsaicint $R_{R}=15.59 \mathrm{~min}$. Identification of the components was performed by means of the respective USP standards.) (Kuzma M. et al., unpublished results)

\subsubsection{Capsaicin USP 29}

Chemical name: 6-Nonenamide, (E)-N-[(4-Hydroxy-3-methoxy-phenyl)methyl]-8-methyl

Formula: $\mathrm{C}_{18} \mathrm{H}_{27} \mathrm{NO}_{3}$

Molecular weight: 305.41

(E)-8-Methyl-N-vanillyl-6-nonenamide CAS-number: 404-86-4

Capsaicin contains not less than 90.0 percent and not more than 110.0 percent of the labeled percentage of total capsaicinoids. The content of capsaicin $(\mathrm{C} 18 \mathrm{H} 27 \mathrm{NO} 3)$ is not less than 55 percent, and the sum of the contents of capsaicin and dihydrocapsaicin $\left(\mathrm{C}_{18} \mathrm{H}_{29} \mathrm{NO}_{3}\right)$ is not less than 75 percent, and the content of other capsaicinoids is not more than 15 percent, all calculated on the dried basis. 
Packaging and storage: Preserve in tight containers, protected from light, and store in a cool place.

Caution: Handle Capsaicin with care. Prevent inhalation of particles of it and prevent its contact with any part of the body.

Solubility: It does not dissolve in water. It well dissolves in alcohols (methanol, ethanol 96\%), ethylacetate and acetonitrile.

Identification: Prepare a test solution of Capsaicin in methanol containing $1 \mathrm{mg} / \mathrm{mL}$. Prepare a Standard solution of USP Capsaicin RS in methanol containing $1 \mathrm{mg} / \mathrm{mL}$. Separately apply $10-\mu \mathrm{L}$ portions of the test solution and the Standard solution to a thin-layer chromatographic plate coated with a 0.25 -mm layer of chromatographic silica gel mixture.

Develop the chromatograms in a solvent system consisting of a mixture of ether and methanol (19:1) until the solvent front has moved about three-fourths of the length of the plate. Remove the plate from the chamber, and allow it to air-dry. Spray the plate with a $0.5 \%$ solution of $2,6-$ dibromoquinone-chlorimide in methanol, allow to stand in a chamber containing ammonia fumes, and examine the chromatograms: the blue color and the $R f$ value of the principal spot obtained from the test solution correspond to those properties of the principal spot obtained from the Standard solution.

Melting range: between $57^{\circ}$ and $66^{\circ}$, but the range between beginning and end of melting does not exceed $5^{\circ}$.

Loss on drying: Dry it in vacuum over phosphorus pentoxide at $40^{\circ}$ for 5 hours: it loses not more than $1.0 \%$ of its weight.

Residue on ignition: not more than $1.0 \%$.

\subsubsection{Stability of capsaicinoids}

Stability of capsaicin and dihydrocapsaicin are different according to the respective MDSD documents: The recommended storage temperature of capsaicin and dihydrocapsaicin are 2-8 ${ }^{\circ} \mathrm{C}$ and $-20^{\circ} \mathrm{C}$, respectively. According to the MDSD sheet, the Sigma-Aldrich Capsaicin natural (pruduct number 21750, CAS number: 404-86-4) (( $65 \%$ capsaicin) should be stored at $2-8{ }^{\circ} \mathrm{C}$.

\section{Literature data only to capsaicin chemistry:}

Kopec et al. tested the stability of $100 \%$ ethanol solution of capsaicin of different concentrations. It was found that the solutions of $4 \mathrm{mM}$ or more concentrated solutions protected from light and stored at $4{ }^{\circ} \mathrm{C}$ have been proved to be stable for a period of 12 months (Kopec et al., 2002).

While investigating stability of a capsaicin containing ointment Jaiarj et al. found the preparation to show higher stability stored at $4{ }^{\circ} \mathrm{C}$ other than at ambient temperature (Jaiarj et al., 2000).

Schweiggert et al. investigated stability of chilli powder. They found that the capsaicin, dihydrocapsaicin and nordihydrocapsaicin content of the samples dropped by 6-11\%. Based 
on their experience it was recommended that paprika (pepper) samples should be heat-treated before processing, in order to reduce the number of microorganisms producing enzymes with peroxidase activity (Schweiggert et al., 2006).

Conclusions: The presence of phenolic hydroxyl gruop and that of the carbon carbon double bond makes capsaicinoids sensitive to oxidation. Accordingly, natural capsaicin should be protected from exposure to light, heat, moisture, and oxidizing agents, wich initiate and/or catalyse the decomposition processes.

\subsubsection{Capsaicin as a pharmacological tool in research}

The term Capsicum refers to the fruit of numerous species of the solanaceous genus Capsicum. The genus name Capsicum is either derived from Greek "Kapso" meaning to bite, referring to its purgency or from the Latin "Capso" or box referring to the fruit pod. Members of the genus vary widely in size, shape, flavor, and much more importantly in purgency. Red hot peppers, also called chilies, paprika, and sweet non-purgent peppers are consumed widely by humans (Maga, 1975; Rozin, 1990, Mózsik et al., 2007). Capsaicin (8-methyl-N-vanillyl-6-nonenamide) is the major purgent ingredient of hot peppers.

\subsubsection{Selective sensory effects of capsaicin}

Apart from being used as a food additive, the compound has wide important pharmacological actions. Capsaicin uniquely selective for mammalian small afferent neurons of dorsal root ganglia $\mathrm{C}$ and $\mathrm{A} \delta$ fibers, a property which led to its use to investigate the role of these afferent fibres in a number of physiological processes (Jancsó and Jancsó-Gábor, 1959; Szolcsányi, 1982; 1984). In the skin the polymodal sensory receptors, chemoreceptors and warm receptors are affected by capsaicin (Szolcsanyi, 1977; 1982; 1993; 1996; Szolcsányi et al., 1994; Bevan and Szolcsányi, 1990; Holzer, 1990; 1991a, 1992 a; 1992 b). Most of these afferents contain substance P (SP) and /or calcitonin gene-related peptide (CGRP) (Holzer, 1991a; 1991 b; Maggi, 1995).

The action of capsaicin expresses itself as an initial short-lasting stimulation that can be followed by desensibilization to capsaicin itself and to other stimuli of afferent sensory neurons. Capsaicin in $n g-\mu \mathrm{g} / \mathrm{kg}$ doses applied to the peripheral or central endings or cell bodies of sensory neurons induces transient excitation of these sensory neurons. In response to stimulation peptide mediators are released from the central and peripheral nerve endings (Szolcsányi, 1984; 1996; Holzer, 1998; 1991a; Maggi, 1995).

In the periphery neuropeptide release exerts local neuroregulatory tissue responses (Szolcsányi, 1984; 1991; 1996). Neuropeptides are stored in sensory vehicles (Gulbekian et al., 1986; Merighi et al., 1988) and are released on stimulation with capsaicin by a $\mathrm{Ca}^{2+}$-dependent manner (Maggi et al., 1989). In this way the peripheral terminals of capsaicin sensitive nerves are not only sensory receptors for conveying impulses in the afferent direction but also effector sites from where mediators are released for neurotransmission (Szolcsányi, 1984; 1996). With large doses $(\mathrm{mg} / \mathrm{kg}$ ) there is an initial stimulation, the duration of which is not yet defined, followed by sensory desensitization (Szolcsányi, 1984; 1993). 
Four response stages of the capsaicin-sensitive primary afferents to capsaicin have been introduced by Szolcsányi (Szolcsányi, 1984; 1985) depending on the dose and duration of exposure to the drug. These are excitation, sensory blocking effect, long-term selective neurotoxic impairment and irreversible cell destruction.

\subsubsection{Mechanism of action of capsaicin on sensory receptors}

To explain the mechanism of these sensory effects of capsaicin it has been hypothetized that in the polymodal nociceptive primary afferent neurons a capsaicin-gated cation channel exists which operates at the peripheral receptive terminals as at the level cell body. Capsaicin exerts its excitatory effect by activation of this cation channel which is permeable to a wide range of cations as $\mathrm{Na}^{+}, \mathrm{K}^{+}$and $\mathrm{Ca}^{2+}$, but not to anions as $\mathrm{Cl}$-(Winter, 1987; Wood et al., 1988). The influx of $\mathrm{Na}^{+}$and $\mathrm{Ca}^{2+}$ induces membrane depolarization which at a certain level, opens tetrotodotoxin-sensitive fast sodium channels at the regenerative region of the sensory receptor and in this way triggers nerve terminal spike and direct stimulation of transmitter release by $\mathrm{Ca}^{2+}$.

Opening of the capsaicin-gated cation channel for shorter or longer periods of time triggers a chain of intracellular events (Bevan and Szolcsányi, 1990). Functional blockage with reversible intracellular molecular changes (stage 2) or neurotoxic degeneration (stages 3 and 4) develop depending on the concentration and the contact time at the site of action on different parts (receptor, axon, cell body, central terminal) of the capsaicin-sensitive primary afferent neurones (Mózsik et al., 2001). Prolonged stimulation and consequently prolonged opening of this cation channel results in osmotic swelling due to intracellular $\mathrm{NaCl}$ accumulation together with intracellular accumulation of $\mathrm{Ca}^{2+}$ at the exposed sites of the nerve terminal which will activates $\mathrm{Ca}^{2+}$-dependent enzymes and impairs mitochondrial functions (Bevan and Szolcsányi, 1990; Chard et al., 1995). Prolonged activation of $\mathrm{Ca}^{2+}$ is therefore the first step in sequence of events ultimately leading to cell death.

In mammals, the long-lasting sensory blocking or neurotoxic effects of capsaicin on primary afferent neurones have been described following topical, perioxonal, or systemic pretreatment in adults or neonates. Autonomic efferent neural mechanisms are not affected by drug (Maggi and Meli, 1988; Szolcsányi, 1982; Holzer, 1991a). This selective sensory blocking effect of capsaicin has been used as an experimental tool for the elimination of the capsaicin-sensitive subset of primary afferent neurones and consequently for identifying those tissue responses that are mediated by capsaicin-sensitive afferent (Szolcsányi, 1996; Mózsik et al. 1997c). The later tissue responses being absent in sensory desensitized animals.

\subsubsection{Capsaicin actions of the gastrointestinal tract in animals}

In their experiments, Makara et al. (1965) found that intragastric capsaicin ( $1 \mathrm{mg} / 0.5 \mathrm{ml}$ volume/ rat) enhanced the development of Shay ulcer at 12 hours. After four consecutive daily doses of reserpine $(1.0 \mathrm{mg} / \mathrm{kg}$, sc.), the above dose of capsaicin given simultaneously enhanced gastric ulceration by the latter. On the other hand, daily administration of paprika oil containing 1.0 $\mathrm{mg} / \mathrm{kg}$ of capsaicin tended to accelerate rather than retard the healing process of reserpineinduced gastric ulcer in rats. 
This was attributed by the authors to the capsaicin-induced local hyperaemia or the carotenoids and other pigments present in paprika oil. When Lee (1963) kept rabbits for 12 months on various diets (high fat,high carbohydrate or high protein) supplemented with large doses of ground hot pepper, ulcers developed in the stomach of all animals and cirrhosis of the liver occurred in animals fed with either high fat or high carbohydrate supplemented with capsaicin.

In the study of Nopanitaya (1974), the effect of capsaicin $(1.0 \mathrm{mg} / \mathrm{kg})$ and its combination with various diets on the morphology of duodenal mucosa of young rats was investigated for period of 28 and 56 days. The author reported ultrastructural alterations in the mitochondria of the absorptive cells of rats fed with low protein diet and also those supplemented with capsaicin. The changes, however, were less pronounced at the $56^{\text {th }}$ day than at $28^{\text {th }}$ day, indicating some sort of adaptation to capsaicin.

It was not known until 1981, that Szolcsányi and Barthó proved, namely that capsaicin protects against experimental gastric ulcer. Introduction of capsaicin into the stomach of pylorusligated rats (Shay)-rats in small doses $(5$ to $50 \mu \mathrm{g})$ and low concentration $(10 \mu \mathrm{g} / \mathrm{mL})$ markedly reduced the ulcer formation at 18 hours later. On the other hand, acute gastric ulceration induced by pylorus-ligation (Shay-ulcer) or acid distension was aggravated in rats desensitized 2 weeks earlier with systematic capsaicin in high doses which selectively impairs capsaicin sensitive sensory nerves.

In capsaicin desensitized rats the aggressive side of the balance remained apparently unchanged since hypersecretion of the pylorus-ligated rats did not differ from that of the controls with respect to volume, $\mathrm{H}^{+}$and pepsin concentration. This suggested that it is the gastric defense mechanism which was impaired in capsaicin desensitized rats. As a result of these data a role of capsaicin sensitive afferents in modulating gastric mucosal defenses was forwarded by the authors. In explaining the nature of this novel gastroprotective action of low dose capsaicin, it was postulated that intragastric capsaicin exerts opposite effects on gastric ulcer formation depending on the concentration in which it is introduced into the gastric lumen. Low concentrations trend to inhibit the development of ulceration and high concentrations promote ulcer formation. Release of vasodilator mediators from capsaicin-sensitive sensory nerve endings with the consequent enhancement of the microcirculation was proposed as the mechanism responsible for the mucosal protective effects on intragastrically administered capsaicin in low concentrations.

These mechanisms suggested a resistance or defense mechanism against ulcer formation working under physiological conditions. In capsaicin desensitized rats, mucosal sensory receptors will be unresponsive to stimuli, and consequently no release of vascular dilator mediators will take place upon challenge with noxious agents. As a result gastric ulcer will be aggravated (Szolcsányi and Barthó, 1981).

These observations and the drawn conclusions were confirmed by other investigators. Intragastric application of capsaicin in small doses has been shown to protect rat gastric mucosa against experimental ulcerations induced by ethanol (Holzer and Lippe, 1988; Esplugues and Whittle, 1989), acidified aspirin (Holzer et al., 1989) and indomethacin (Gray 
et al., 1994). Furthermore, after desensitization rats exhibited more severe gastric mucosal damage than their sensory intact controls in response to chemical challenge with ethanol (Holzer and Lippe, 1988; Lippe et al., 1989; Esplugues and Whittle, 1990; Esplugues et al., 1992; Pabes et al., 1993), cysteamine (Holzer and Samettz, 1986; Gray et al., 1994), platelet activating factor (Esplugues et al., 1989; Pique et al., 1990) and endothelin-1 (Whittle and Lopez-Belmonte, 1991).

Capsaicin desensitation performed 6 days prior to cold restraint stress, however, was reported to have a little effect on gastric ulcer formation. Only the number of lesions was higher in capsaicin treated rats restrained for 3 hours (Dugani and Glavin, 1986). Acute intragastric capsaicin $(40 \mathrm{mg} / \mathrm{kg})$ followed immediately by stress resulted in significantly more frequent and more severe ulceration (Dugani and Glavin, 1985). Cysteamine-induced duodenal ulcers (Holzer and Sametz, 1986) and gastric mucosal damage evoked by $0.6 \mathrm{M} \mathrm{HCl}$ (Takeuchi et al., 1994) were not changed after capsaicin desensitization.

Studies have shown that gastric mucosal barrier disruption is accompanied with an increase of gastric mucosal blood flow (GMBF), which appears to be triggered by $\mathrm{H}^{+}$rediffused through the breached mucosal defenses (Bruggeman et al., 1979; Starlinger et al., 1981a). Such an increase in GMBF is through to be a defense mechanism, whereby the increased blood flow prevents the accumulation of injurious concentration of $\mathrm{H}^{+}$in the submucosa. Capsaicinsensitive nerves which are unduly sensitive to $\mathrm{H}^{+}$(Bevan and Yeats, 1991) have been shown to respond to $\mathrm{H}^{+}$back-diffused through breached mucosal defenses and to signal for an increase in gastric mucosal blood flow (Holzer et al., 1991b). This further strengthened the role of these nerves in maintaining mucosal integrity. Holzer el al. (1991a, b) postulated that the acidinduced mucosal hyperaemia results from local axon reflex between collaterals of afferent nerve fibres within the gastric wall. Li et al. (1992) found that gastric mucosal hyperaemia in response to perfusion of the rat stomach with $0.15 \mathrm{M} \mathrm{HCl}$ in $15 \mathrm{v} / \mathrm{v}$ ethanol to be completely blocked by close arterial infusion of a hCGRP receptor antagonist.

Lippe and Holzer (1992) reported in rats that, N-nitro-L-arginine methyl ester (L-NANE) (an inhibitor of endothelium derived nitric oxide formation) depressed the increase in GMBF produced by gastric perfusion with ethanol diluted in $0.15 \mathrm{M} \mathrm{HCl}$ The loss of $\mathrm{H}^{+}$from the lumen under these circumstances was also markedly enhanced by L-NAME. Considerable controversy still exists in the literature in regards to the mediators of the hyperaemic response to back-diffusion of acid (Whittle, 1977; Ritchie, 1991; Gislason et al., 1995).

The involvement of capsaicin-sensitive sensory nerves in repair mechanisms of the injured mucosa have also been investigated. In anaesthetized rats, $180 \mathrm{~min}$ after exposure to $50 \mathrm{v} / \mathrm{v}$ ethanol, rapid repair of the injured mucosa (assessed by reduction of deep mucosal damage and partial reepitheliazition of the denuted surface) was reported to be similar in sensory denervated and sensory intact groups. This suggested that nociceptive neurones control mechanisms of defense against acute gastric mucosal injury, but they are not required for the rapid repair of the injured mucosa (Pabst et al., 1993). On contrary, sensory differention delayed healing of gastric ulcers provoked in rats by $0.6 \mathrm{M} \mathrm{HCl}$. 
In addition capsaicin-desensitized not sensory intact animals showed no hyperaemic responses in response to intragastric instillation of $50 \mathrm{mM} \mathrm{HCl}$. The conclusion was that capsaicinsensitive sensory nerves contribute to healing of gastric ulcer (lesions) by mediating the hyperaemic responses associated with acid back-diffusion following injury (Takeuchi et al., 1994). Similarly sensory denervated rats showed marked increased area of acetic acid-induced ulceration at 1 and 2 weeks following the acetic acid injection indicating that the sensory function adversely affected the healing of gastric ulcer (Tramontana et al., 1994).

Capsaicin exerts protective effects on the chemical-induced mucosal injury not only in the stomach, but also in the colon. Evangelista and Meli (1989) found that systemic capsaicin neonatal pretreatment enhanced trinitrobenzene sulfonic acid-induced colitis (one week) in rats. This pretreatment, however, had no acute affect ( 24 hours) on colitis caused by trinitrobenzene sulfonic acid, ethanol or acetic acid.

Reinshagen et al. (1994) employed systemic capsaicin pretreatment as a tool to investigate the role of sensory nerves in an immune-complex model of colitis in rabbits. They found that capsaicin pretreatment per se caused no histological inflammation. Meanwhile, colitis was more severe in sensory denervated state than in sensory intact rabbits. The increase in ulcer index and neutrophyl infiltration was more marked in the capsaicin pretreated control group at both 48 and 96 hours.

The difference in neutrophyl infiltration between the two groups was, however, more marked at 48 than at 96 hours (Reinshagen et al., 1994). Endoh and Leung (1990) reported that topical capsaicin application protected against acetic acid-induced colitis. In the trinitrobenzene sulfonic acid-induced colitis rat model, however, only partial and transient protective effect was seen by Goso et al. (1993) after topical capsaicin administration. Co-administration of 640 $\mu \mathrm{M}$ capsaicin reduced the ulcerative area from $91 \%$ to $64 \%$ only when colon was examined 1 hour later. An approximatically 8 fold higher dose of capsaicin $(5000 \mu \mathrm{M})$ yielded similar protection, while $100 \mu \mathrm{M}$ had no protective effect. No protection by capsaicin was however seen when the colon was examined 24 hours after noxious challenge.

\subsubsection{Capsaicin-sensitive sensory nerves and gastric acid secretion}

Several studies in rats have indicated the involvement of capsaicin-sensitive sensory nerves in the regulation of gastric acid secretion; however, contradictory data were reported. In most studies, the indirect approach through functional ablation of capsaicin-sensitive afferent nerves with systemic neonatal (Evangelista et al., 1989; Esplugues et al., 1990), adult (Alföldi et al., 1986; 1987; Dugani and Glavin, 1986; Robert et al., 1991) or peripheral capsaicin (Raybould and Taché, 1989) treatment was used as a tool to investigate the role of capsaicinsensitive afferent nerves in the regulation of gastric acid secretion.

Adult rats treated with systemic capsaicin $(60 \mathrm{mg} / \mathrm{kg}$, sc.) showed depressed pentagastrinstimulated gastric acid secretion (Dugani and Glavin, 1986). On the contrary, adult systemic capsaicin pretreatment with $300 \mathrm{mg} / \mathrm{kg}$ sc. did not modify gastric acid secretion elicited by pentagastrin, carbachol or by small dose of histamine $(0.1 \mathrm{mg} / \mathrm{kg})$. However, the gastric acid secretory response to 0.5 and $5.0 \mathrm{mg} / \mathrm{kg}$ histamine was greatly reduced in capsaicin desensi- 
tized rats. It was suggested that the histamine-induced increase in gastric acid secretion involves a capsaicin-sensitive mechanism, while these mechanisms are not required for pentagastrin or cholinergic stimulation of gastric acid secretion (Alföldi et al., 1986; 1987).

Similar data were reported by Raybould and Taché (1989) using topical capsaicin application into the cervical vagus. The gastric acid secretory response to distension ( $5 \mathrm{ml}$ for $6 \mathrm{~min}$ ) was reduced in capsaicin-treated rats. This mechanism by which capsaicin-sensitive vagal afferent fibres play a role in the secretory response to histamine was explained by histamine acting in part by increasing vagal C-fibres discharge resulting in a vago-vagal reflex increase in gastric acid secretion or by that histamine stimulates vagal afferent $\mathrm{C}$-fibres resulting the release of peptides from sensory nerves terminals.

A peptide increasing gastric acid secretion and would be localized in vagal afferent nerves has not been identified. In contrast that adult rats treated with systemic capsaicin ( $300 \mathrm{mg} / \mathrm{kg}$, sc.) at neonate age did not show any reduction in their gastric acid secretory to histamine, pentagastrin or carbachol, while acid secretion in response to distension was abolished. On the other hand, capsaicin desensitation (neonatal treatment) substantially reduced the gastric acid secretion to 2-deoxy-D-glucose (Evangelista et al., 1989), while it did not modify when stimulated by insulin (Esplugues et al., 1990).

The conflicting observations regarding the effect of capsaicin continue to be seen when it was given into the stomach. It was reported that intraduodenal (but not intragastric) instillation of capsaicin (1.0 mg in $2 \mathrm{ml}$ saline solution) in pylorus-ligated rats induced a significant rise in total acidity 12 hours later (Makara et al.,1965). The results with capsaicin indicated in some meaning contradictory results in the gastrointestinal tract mentioned above. Relatively same attention was played to the applied doses of capsaicin. Szolcsányi and Barthó (1981), however, emphasized well that capsaicin protects against the chemical induced gastric ulcer formation, when the capsaicin was given in 5 and $50 \mu \mathrm{g}$ doses $(10 \mu \mathrm{g} / \mathrm{mL}$ concentration) intragastrically, meanwhile capsaicin in high dose aggravated the ulcer formation by the induction of desensitazion.

Very systematic observations were carried out by us with capsaicin on dependence of its concentration (or pretreatment produced desensitization of capsaicin sensitive afferent nerves) on different experimental models (aspirin, $\mathrm{HCl}$, indomethacin, ethanol, cysteamine). The changes of gastric acid secretion, gastric mucosal damage, gastric $\mathrm{H}^{+}$back-diffusion, gastric mucosal blood flow were measured and calculated, when the capsaicin was applied in small doses and in high doses producing desensitization (Mózsik et al., 1997c). The results of these observations clearly demonstrated that: 1 . capsaicin, given in small doses, dose-dependently inhibited all of the parameters in all experimental models; 2.the gastric mucosal protective effects of capsaicin remained at the level of other drugs (acting on efferent nerves, eg. atropine, and cimetidine or at topically such as sucralfate, retinoids). Consequently, the capsaicin enhanced the other drug-induced gastric mucosal protective effects (Mózsik et al., 1997c); 3. after denervated states of capsaicin-sensitive afferent nerves (produced by pretreatment of high dose of capsaicin) the gastric mucosal lesion formation was enhanced. These results offered to conclude that the gastric mucosal protective effects can be obtained only capsaicin, when it is given in small doses, however this gastric mucosal protective effect of capsaicin can 
not be obtained by the application of higher doses (Abdel-Salam et al., 1994, 1995a, 1995b, 1995c, 1995d, 1995e, 1995f, 1995g, 1996a, 1996b, 1997a, 1997b, 1997c, 1997d; Mózsik et al.,1993, 1996a, 1996b, 1997a, 1997b, 1997)

\subsubsection{Molecular-pharmacological studies}

The molecular pharmacological observations were carried out (and calculated based on the dose-response curves of drugs) with capsaicin, atropine, cimetidine, omeprazole, $\mathrm{PGI}_{2}$, vitamin A, $\beta$-carotene, studying their effects on the gastric acid secretion in 2 and 4 hours pylorusligated rats alone, or in combination of betanechol $(7.6$ and $15.4 \mathrm{nmol} / \mathrm{kg})$, histamine (2.7 and $13.6 \mu \mathrm{mol} / \mathrm{kg}$ ) and pentagastrin $(65.1$ and $325.6 \mathrm{nmol} / \mathrm{kg})$ and on the gastric mucosal damage produced by intragastrically applied ethanol, $\mathrm{HCl}$, acidified aspirin and subcutaneously applied indomethacin (alone and in combinated with application of 7.6 and $15.2 \mu \mathrm{mol} / \mathrm{kg}$ betanechol, 13.6 and $54.3 \mu \mathrm{mol} / \mathrm{kg}$ histamine and 6.51 and $325.6 \mathrm{nmol} / \mathrm{kg}$ pentagastrin) (calculated the number and severity of gastric mucosal damage) in rats (Figures 4-7, Tables 1-7).

The doses of the necessary for the producing $50 \%$ inhibition on the gastric acid secretion and gastric mucosal damage were calculated in molar values/kg body weight $\left(\mathrm{ED}_{50}\right)$.

The values for affinity $(\mathrm{pD})$ and intrinsic ( $\alpha$-values) were calculated according to standard procedures employed in molecular pharmacology (Csáky, 1969). The values of the $\mathrm{pD}_{2}$ (necessary dose to inhibit the gastric acid secretion and gastric mucosal damage in 50\%) and $\mathrm{pA}_{2}$ (necessary dose to produce $50 \%$ in gastric acid secretion and on gastric mucosal damage) were calculated from the affinity and intrinsic activity curves.

The doses of drugs (compounds) were calculated as molar values for the determination of their biological effects. The affinity $\left(\mathrm{pD}_{2}\right.$ values) and intrinsic activity ( $\alpha$-values) are shown as molar values. The intrinsic activity of atropine $(\alpha)$ was taken as 1.00 for comparing the effects of agents on gastric acid secretion and gastric mucosal damage (Figures 4-7, Tables 1-7) (Mózsik et al., 2006).

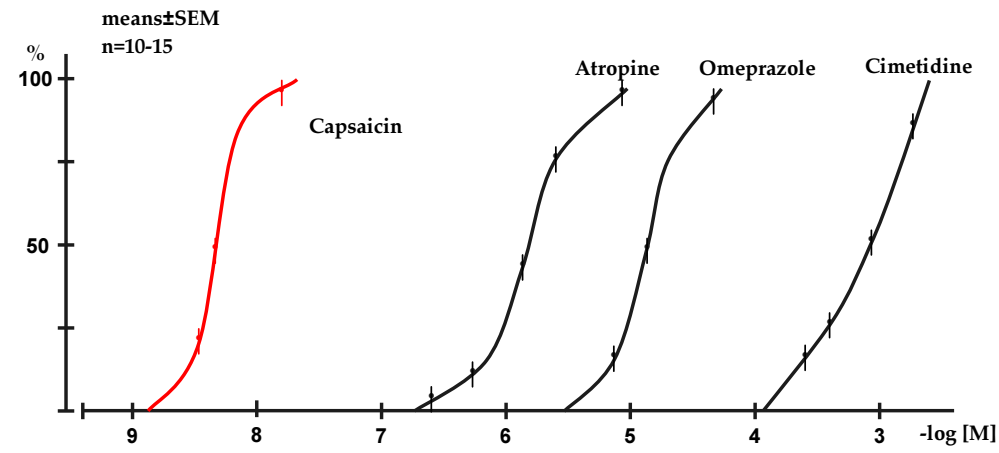

Figure 4. Affinity curves for different drugs, compounds inhibiting the gastric acid secretion of $4 \mathrm{~h}$ pylorus-ligated rats (Mózsik et al., 2006b). For further details of the observations see the cited paper. 


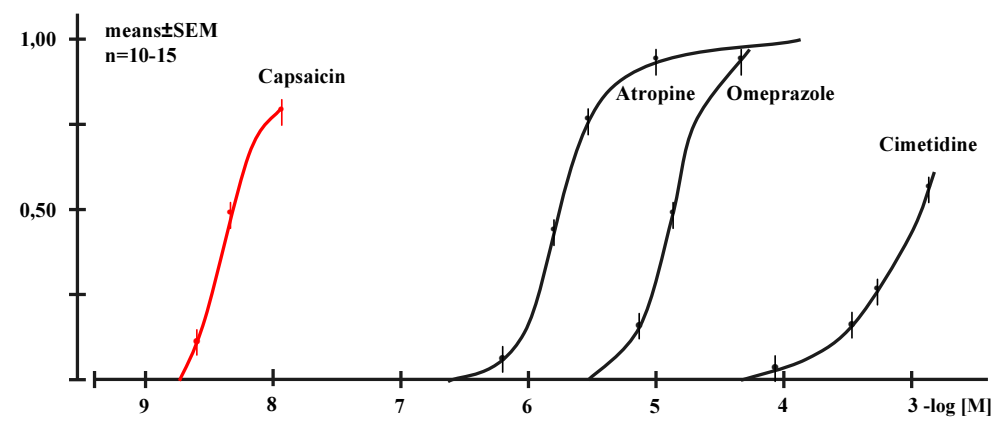

Figure 5. Intrinsic affinity curves for different drugs, compounds inhibiting the gastric acid secretion of $4 \mathrm{~h}$ pylorusligated rats, which were expressed in relation to that of atropine $(1,00)$ ( $a_{\text {atropine }}$ ) (Mózsik et al., 2006b). For further details of the observations see the cited paper.

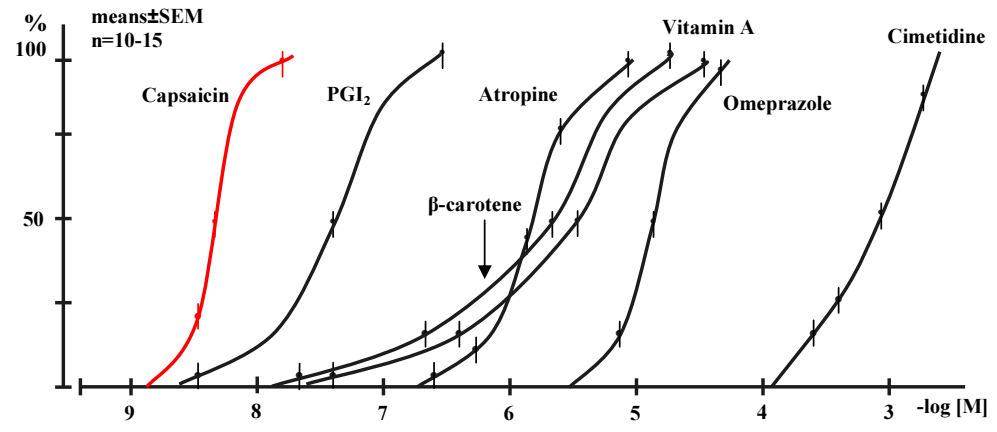

Figure 6. Affinity curves for different drugs, compounds inhibiting the gastric mucosal damage produced by various chemical agents in rats (Mózsik et al., 2006b). For further details of the observations see the cited paper.

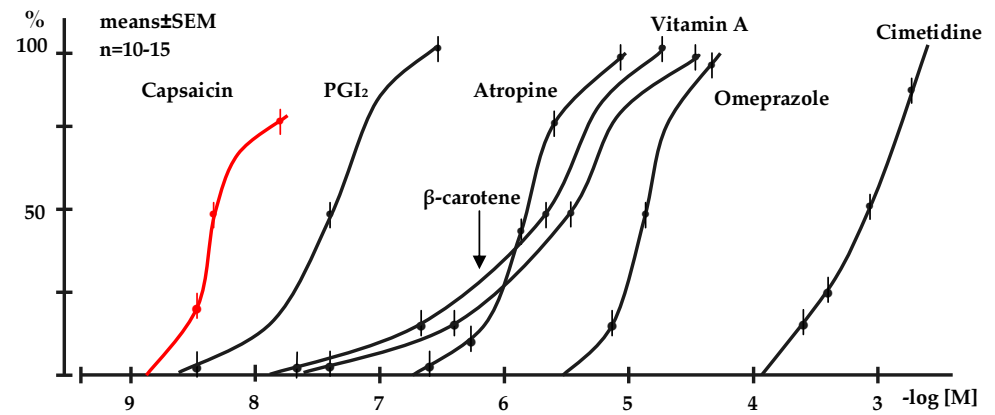

Figure 7. Intrinsic activity curves for different drugs, compounds inhibiting the gastric mucosal damage produced by chemical agents which were expressed relative to that of atropine $\left(a_{\text {atropine }}=1,00\right)$ in rats (Mózsik et al., 2006b). For further details of the observations see the cited paper. 


\begin{tabular}{|c|c|c|}
\hline Compounds & Models & $\mathrm{ED}_{50}$ values \\
\hline Capsaicin & $2 \mathrm{~h}$ pylorus ligated rats & $3.27 \mathrm{nmol} / \mathrm{kg}$ \\
\hline Capsaicin & $4 \mathrm{~h}$ pylorus ligated rats & $3.27 \mathrm{nmol} / \mathrm{kg}$ \\
\hline RTX & $4 \mathrm{~h}$ pylorus ligated rats & $0.954 \mathrm{nmol} / \mathrm{kg}$ \\
\hline
\end{tabular}

Table 1. Inhibitory effects of capsaicin and resiniferatoxin on basal gastric acid secretion in rats. *

\begin{tabular}{lll}
\hline Compounds & Models & ED $_{50}$ values \\
\hline Capsaicin & 1 h pylorus ligated rats + betanechol $(7.6 \mu \mathrm{mol} / \mathrm{kg})$ & $0.954 \mathrm{nmol} / \mathrm{kg}$ \\
\hline Capsaicin & 1 h pylorus ligated rats + betanechol $(15.2 \mu \mathrm{mol} / \mathrm{kg})$ & $0.954 \mathrm{nmol} / \mathrm{kg}$ \\
\hline Capsaicin & 1 h pylorus ligated rats+ histamine $(13.6 \mu \mathrm{mol} / \mathrm{kg})$ & $0.954 \mathrm{nmol} / \mathrm{kg}$ \\
\hline Capsaicin & 1 h pylorus ligated rats + histamine $(54.3 \mu \mathrm{mol} / \mathrm{kg})$ & $0.954 \mathrm{nmol} / \mathrm{kg}$ \\
\hline Capsaicin & 1 h pylorus ligated rats + pentagastrin $(65.1 \mathrm{nmol} / \mathrm{kg})$ & $0.954 \mathrm{nmol} / \mathrm{kg}$ \\
\hline Capsaicin & 1 h pylorus ligated rats pentagastrin $(325.6 \mathrm{nmol} / \mathrm{kg})$ & $0.954 \mathrm{nmol} / \mathrm{kg}$ \\
\hline RTX & 4 h pylorus ligated rats + betanechol $(7.6 \mathrm{nmol} / \mathrm{kg})$ & $0.954 \mathrm{nmol} / \mathrm{kg}$ \\
\hline RTX & 4 h pylorus ligated rats+ betanechol $(15.2 \mathrm{nmol} / \mathrm{kg})$ & $0.954 \mathrm{nmol} / \mathrm{kg}$ \\
\hline RTX & 4 h pylorus ligated rats + histamine $(2.7 \mu \mathrm{mol} / \mathrm{kg})$ & $0.954 \mathrm{nmol} / \mathrm{kg}$ \\
\hline RTX & 4 h pylorus ligated rats + histamine $(13.6 \mu \mathrm{mol} / \mathrm{kg})$ & $0.954 \mathrm{nmol} / \mathrm{kg}$ \\
\hline RTX & 4 h pylorus ligated rats + pentagastrin $(65.1 \mathrm{nmol} / \mathrm{kg})$ & $0.954 \mathrm{nmol} / \mathrm{kg}$ \\
\hline RTX & 4 h pylorus ligated rats pentagastrin $(325.6 \mathrm{nmol} / \mathrm{kg})$ & $0.954 \mathrm{nmol} / \mathrm{kg}$ \\
\hline
\end{tabular}

* (Mózsik et al., 2006b)

Table 2. Inhibitory effects of resiniferatoxin on stimulated gastric acid secretion in rats. *

\begin{tabular}{lll}
\hline Compounds & Models & ED $_{50}$ values \\
\hline Capsaicin & 0.6 M HCl $(2 \mathrm{ml})(4 \mathrm{~h})$ & $0.135 \mu \mathrm{mol} / \mathrm{kg}$ \\
\hline Capsaicin & aspirin $(200 \mathrm{mg} / \mathrm{kg})+0.15 \mathrm{M} \mathrm{HCl}(4 \mathrm{~h})$ & $0.13 \mu \mathrm{mol} / \mathrm{kg}$ \\
\hline Capsaicin & ethanol $(96 \% \mathrm{in} 1 \mathrm{ml})(1 \mathrm{~h})$ & $0.13 \mu \mathrm{mol} / \mathrm{kg}$ \\
\hline Capsaicin & 4 h pylorus ligated rats+ IND $(20 \mathrm{mg} / \mathrm{kg})$ & $1.98 \mathrm{nmol} / \mathrm{kg}$ \\
\hline RTX & 4 h pylorus ligated rats + IND $(20 \mathrm{mg} / \mathrm{kg})$ & $0.95 \mathrm{nmol} / \mathrm{kg}$ \\
\hline Capsaicin & 4 h pylorus ligated rats +IND $(20 \mathrm{mg} / \mathrm{kg})+0.15 \mathrm{~N} \mathrm{HCl}(2 \mathrm{ml})$ & $0.25 \mu \mathrm{mol} / \mathrm{kg}$ \\
\hline RTX & 4 h pylorus ligated rats + IND $(20 \mathrm{mg} / \mathrm{kg})+0.15 \mathrm{~N} \mathrm{HCl}(2 \mathrm{ml})$ & $0.95 \mathrm{nmol} / \mathrm{kg}$ \\
\hline Capsaicin & 4 h pylorus ligated rats + IND $(20 \mathrm{mg} / \mathrm{kg}) 0.3 \mathrm{~N} \mathrm{HCl}(2 \mathrm{ml})$ & $2.78 \mu \mathrm{mol} / \mathrm{kg}$ \\
\hline RTX & 4 h pylorus ligated rats + IND $(20 \mathrm{mg} / \mathrm{kg}) 0.3 \mathrm{~N} \mathrm{HCl}(2 \mathrm{ml})$ & $0.15 \mu \mathrm{mol} / \mathrm{kg}$ \\
\hline
\end{tabular}

*(Mózsik et al., 2006b)

Table 3. Protective effects of capsaicin and resiniferatoxin on gastric mucosal damage caused by exogenous agents in rats.* 


\begin{tabular}{llc}
\hline Compounds & Models & ED $_{50}$ values \\
\hline RTX & 4 h pylorus ligated rats + bethanechol $(7.6 \mu \mathrm{mol} / \mathrm{kg})+$ IND $(20 \mathrm{mg} / \mathrm{kg})$ & $0.20 \mu \mathrm{mol} / \mathrm{kg}$ \\
\hline RTX & $4 \mathrm{~h}$ pylorus ligated rats+ bethanechol $(15.2 \mu \mathrm{mol} / \mathrm{kg})+\mathrm{IND}(20 \mathrm{mg} / \mathrm{kg})$ & $0.20 \mu \mathrm{mol} / \mathrm{kg}$ \\
\hline RTX & 4 h pylorus ligated rats+ histamine $(13.6 \mu \mathrm{mol} / \mathrm{kg})+\mathrm{IND}(20 \mathrm{mg} / \mathrm{kg})$ & $0.13 \mu \mathrm{mol} / \mathrm{kg}$ \\
\hline RTX & $4 \mathrm{~h}$ pylorus ligatedrats + histamine $(54.3 \mu \mathrm{mol} / \mathrm{kg})+\mathrm{IND}(20 \mathrm{mg} / \mathrm{kg})$ & $0.13 \mu \mathrm{mol} / \mathrm{kg}$ \\
\hline RTX & $4 \mathrm{~h}$ pylorus ligated rats+ pentagastrin $(6.51 \mathrm{nmol} / \mathrm{kg})+\mathrm{IND}(20 \mathrm{mg} / \mathrm{kg})$ & $0.13 \mu \mathrm{mol} / \mathrm{kg}$ \\
\hline RTX & $4 \mathrm{~h}$ pylorus ligated rats+ pentagastrin $(325.6 \mathrm{nmol} / \mathrm{kg})+\mathrm{IND}(20 \mathrm{mg} / \mathrm{kg})$ & $0.13 \mu \mathrm{mol} / \mathrm{kg}$ \\
\hline
\end{tabular}

*(Mózsik et al., 2006b)

Table 4. Protective effects of resiniferatoxin on gastric mucosal damage caused by exo-, and endogenous agents in rats.*

\begin{tabular}{llll}
\hline Compounds & $\mathbf{p D}_{\mathbf{2}}$ & Intrinsic activity & $\mathbf{p A}_{\mathbf{2}}$ \\
\hline Capsaicin & 8.48 & 0.76 & 8.50 \\
\hline Atropine & 5.75 & 1.00 & 5.80 \\
\hline Cimetidine & 3.00 & 0.64 & 3.20 \\
\hline Omeprazole & 4.88 & 1.00 & 4.90 \\
\hline
\end{tabular}

* For further information, see Figures 1 and 2 (Mózsik et al., 2006b)

Table 5. The $\mathrm{pD}_{2}$, intrinsic activity $\left(a_{\text {atropine }}=1.00\right)$ and $\mathrm{pA}_{2}$ values for different drugs (compound) inhibiting the gastric acid outputs in $4 \mathrm{~h}$ pylorus-ligated rats.*

$\begin{array}{ll}\text { Vitamin A: } & 3.49 \times 10-8-3.49 \times 10-5(0.01-10.0 \mathrm{mg} / \mathrm{kg}): \text { no inhibitory action on the gastric acid secretion } \\ \text { B-carotene: } & 1.86 \times 10-8-1.86 \times 10-5(0.01-10.0 \mathrm{mg} / \mathrm{kg}): \text { no inhibitory action on the gastric acid secretion } \\ \text { PGI }_{2}: & 2.8 \times 10-9-1.42 \times 10-8(1.0-5.0 \mu \mathrm{g} / \mathrm{kg}): \text { no inhibitory action on the gastric acid secretion } \\ \text { PGE }_{2}: & 1.33-1.99 \times 10-7(50.0-150.0 \mu \mathrm{g} / \mathrm{kg}): \text { no inhibitory action on the gastric acid secretion }\end{array}$

*(Mózsik et al., 2006b)

Table 6. Dose ranges of the tested nutritional compounds (vitamin $A$ and $\beta$-carotene), $\mathrm{PGI}_{2}$ and $\mathrm{PGE}_{2}$ on the gastric acid secretion of $4 \mathrm{~h}$ pylorus-ligated rats, without presence any inhibitory actions. * 


\begin{tabular}{llll}
\hline Compounds & $\mathbf{p D}_{2}$ & Intrinsic activity & $\mathbf{p A}_{\mathbf{2}}$ \\
\hline Capsaicin & 8.48 & 0.76 & 8.50 \\
\hline$\left.\mathrm{PG}\right|_{2}$ & 7.45 & 1.00 & 7.44 \\
\hline Atropine & 5.75 & 1.00 & 5.80 \\
\hline Cimetidine & 3.00 & 0.64 & 3.20 \\
\hline Omeprazole & 4.88 & 1.00 & 4.90 \\
\hline Vitamin A & 5.45 & 1.00 & 5.44 \\
\hline -carotene & 5.73 & 1.00 & 5.73 \\
\hline
\end{tabular}

* For further information, see Figures 4-7. (Mózsik et al., 2006b)

Table 7. The $\mathrm{pD}_{2}$, intrinsic activity $\left(\mathrm{a}_{\text {atropine }}=1.00\right)$ and $\mathrm{pA}_{2}$ values for different drugs, compounds inhibiting the gastric mucosal damage produced by chemical agents in rats. *

The results obtained demonstrated the following conclusions:

1. Only the values of $\mathrm{pD}_{2}$ and $\mathrm{pA}_{2}$ (expressed in [-] molar doses) can be used for the evaluation of physiological and pharmacological regulations of the target organ(s) in animalexperiment(s) (Mózsik et al., 2006).

2. The following $\mathrm{pD}_{2}\left(\mathrm{ED}_{50} \%\right)$ values were obtained for the different drugs (compounds) for actions in inhibiting gastric acid secretion: atropine 5.75; omeprazole 4.88; cimetidine 3.00; capsaicin 8.50, whereas no effects were observed with $\mathrm{PGI}_{2}$, vitamin $\mathrm{A}$ and $\beta$-carotene.

3. The intrinsic activity values $\left(\alpha_{\text {atropine }}=1.00\right)$ obtained in relation to atropine were 0.64 for cimetidine, 0.75 for capsaicin, 1.00 for omeprazole; no effects were observed for $\mathrm{PGI}_{2}$, vitamin $\mathrm{A}$ and $\beta$-carotene.

4. The following $\mathrm{pD}_{2}\left(\mathrm{ED}_{50}\right)$ values were obtained for he different drugs or compounds inhibiting the gastric mucosal damage produced by chemicals: capsaicin $8.48, \mathrm{PGI}_{2} 7.45$, atropine 5.75, cimetidine 3.00, omeprazole 4.88, vitamin A 5.45 and $\beta$-carotene 5.73.

5. The intrinsic activity values $\left(\alpha_{\text {atropine }}=1.00\right)$ were obtained: capsaicin 0.76 , cimetidine 0.64 , of other components were 1.00 on the gastric mucosal damage.

6. The values of $\mathrm{pA}_{2}$ values were obtained as follows: capsaicin 8.50, $\mathrm{PGI}_{2} 7.44$, atropine 5.80, cimetidine 3.00, omeprazole 4.90, vitamin A 5.44 and $\beta$-carotene 5.70.

The results of these molecular pharmacological observations clearly indicated (proved) that the capsaicin-sensitive afferent nerves have essential role both in the regulation of gastric acid secretion and in the defense of the gastric mucosal damage produced by the different chemical agents. It has been emphasizing that the capsaicin exerts the gastric acid inhibitory and the 
gastric mucosal protective effect in smaller molar concentrations than other compounds (atropine, cimetidine, omeprazole and other compounds without any gastric acid inhibitory effects). That is a clear explanation for why the essential role of capsaicin-sensitive afferent nerves is deeply emphasized in the physiological and pharmacological regulation of gastrointestinal tract.

\subsubsection{Capsaicin actions in human healthy subjects and in patients with different gastrointestinal disorders}

Early work regarding the effect of peppers on the human stomach has yielded conflicting results. In peptic ulcer patients, Schneider et al., (1956) studied the influence of a variety of spices on sensation of pain and healing peptic ulcer. Ulcer patients placed on established treatment with anticholinergics and diet were given different purgent species in capsules that contained the average quantity habitually consumed by Americans with three of their daily meals for six weeks. With the exception of black pepper, which resulted in distressing pain after one or two days, none of the tested spices have modified pain sensation or delayed healing of ulcers.

In other study, instillation of red chilli powder was reported to be associated with significant increase of DNA from gastric aspirates (Desai et al., 1973). Viranuvatti et al. (1972) studies the local effect of capsicum in twenty human subjects by instillation a 3\% capsicum solution through an intragastric tube or via the lumen of gastrofiberscope. There was no change in 13 cases, oedema and/or hyperaemia developed in three cases, haemorrhagic spots occurred in another three cases and bleeding occurred in one case.

Capsaicin was reported to increase the gastric acidity in human subjects receiving intragastrically aqueous suspensions from hot peppers (Berkessy, 1934; Varga, 1936; Ketusinh et al., 1966; Solanke, 1973). Solanke (1973) studied the effect of red pepper suspension ( $200 \mathrm{ml}$ of $4 \%$ solution) instilled through a nasogastric tube on gastric acid secretion in patients with duodenal ulcer and non-duodenal ulcer. Patients were allocated into two treatments: fresh red pepper suspension and a red pepper suspension with $\mathrm{pH}$ adjusted to 7.4 with $0.01 \mathrm{~N}$ sodium hydroxyde. They found a significant increase in gastric acid secretion after treatment with either form of the red pepper suspension. Many other observations were carried out in human beings with different extractions of chilli, paprika. Consequently we have no scientifically well controlled human studies on the applied doses of capsaicin. On the other hand, these studies were carried out in sporadic pathways.

The Good Clinical Practice (GCP) was introduced in the clinical research of human beings and patients.

From the years of 1997, the clinical studies with capsaicin were carried out in prospective and randomized, multiclinical conditions as those had been officially accepted in the multiclinical pharmacological studies in all over the World.

The following observation facts were accepted in these human observations: 
1. The pure capsaicin (Sigma, USA, later on Sigma-Aldrich, USA) was used in the studies with healthy human subjects and in patients with different diseases (instead of different extractions of different capsaicin containing plants),

2. The clinical observations were carried out according to the medical laws of clinical pharmacology (in random allocation and in prospective and randomized studies),

3. The clinical observations were carried out according to the criteria of GCP,

4. All of the persons participating received all doses of capsaicin in random allocation.

We have to emphasize that the classical pharmacological studies were carried out with capsaicin obtained from the firm of Sigma (USA), or later from Sigma-Aldrich (USA).

The main results of the human multiclinical pharmacological studies with capsaicin:

1. The capsaicin (in range of 100 to $800 \mu \mathrm{g}$ dose orally given to each person) dose-dependently inhibited the gastric basal acid output (BAO) in healthy human subjects (Fig. 8.) (Mózsik et al., 1999; Mózsik et al., 2005).

2. The capsaicin dose-dependently enhanched the the gastric transmucosal potential difference (GTPD) in healthy human subjects (Fig. 9) (Mózsik et al., 2005).

3. The ethanol-induced decrease of GTPD can be dose-dependently reversed by topical application of capsaicin (given it in doses of 100, 200,400 and $800 \mu \mathrm{g}$ orally) (Fig. 10) (Mózsik et al., 2005).

4. The indomethacin ( $3 \times 25 \mathrm{mg}$ /day given orally,plus $25 \mathrm{mg}$ given immediately before measuring of gastric blood losing) produced a significant increase of gastric microbleeding in comparison to control (untreated) conditions (Fig. 11) (Mózsik et al., 2005; Mózsik et al., 2006).

5. The extent basal and indomethacin-induced gastric microbleeding unchanged before and after weeks treatment with capsaicin (3x 400 g/ person/day) (Mózsik et al., 2005; Mózsik et al., 2007).

6. The dose-dependent gastroprotective effect of capsaicin on the indomethacin-induced gastric microbleeding remained the same after two weeks ( $3 \times 400 \mu \mathrm{g}$ given orally /day/ person) of capsaicin treatment (Mózsik et al., 2005; Mózsik et al., 2006).

These observations proved that the capsaicin (dose-dependently) prevents the ethanoland indomethacin-induced gastric mucosal damage in healthy human subjects (Mózsik et al., 2005; Mózsik et al., 2007) before and after two weeks treatment with capsaicin (3x 400 $\mu \mathrm{g}$ orally /day/ person) (Mózsik et al., 2005; Mózsik et al., 2006), however, we have to emphasize that the indomethacin $(3 \times 25 \mathrm{mg}$ given orally, plus $25 \mathrm{mg}$ given immediately before the measuring of gastric microbleeding) produced the same extent of gastric microbleeding. We also have to emphasize that the extent of baseline of gastric microbleeding remained the same before and after two week treatment with capsaicin (Mózsik et al., 2005; Mózsik et al., 2007). 


\section{Effect of capsaicin on gastric basal acid output (BAO) in healthy human subjects}

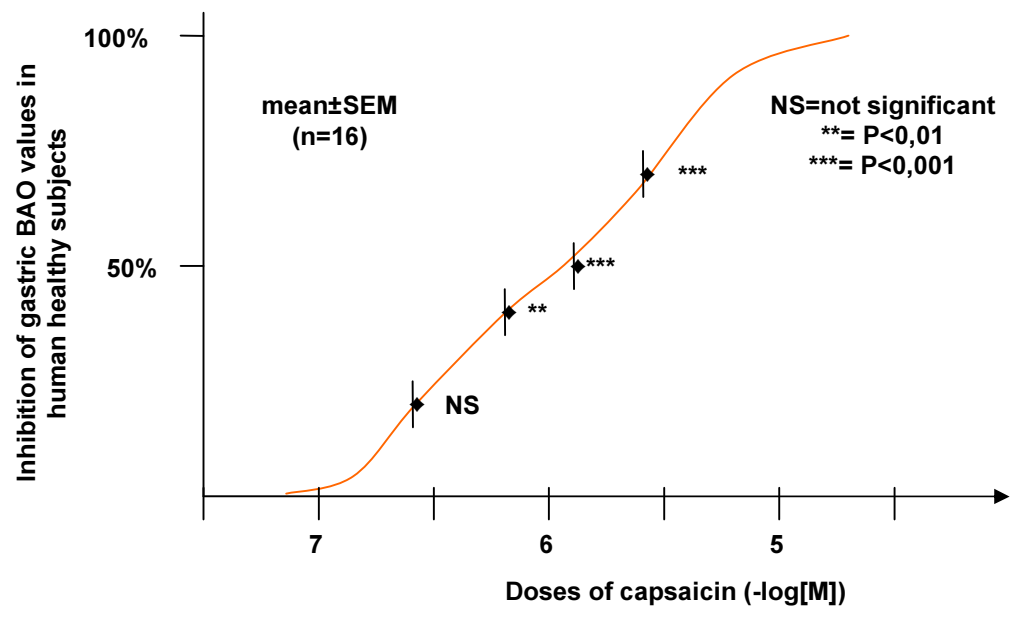

Figure 8. Dose-response curve of capsaicin on the gastric basal acid output (BAO) in healthy human subjects (Mózsik Gy. et al., 2005). The determination was carried out at one hour after the beginning of the human observations.

\section{Effect of capsaicin on GTPD (- $\Delta \mathbf{m V})$ in healthy human subjects}

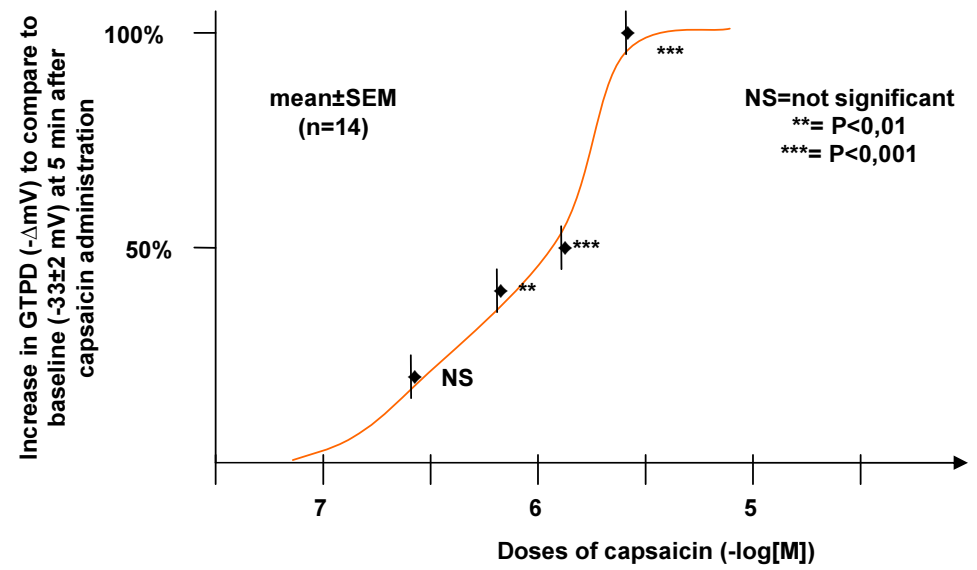

Figure 9. Dose-response curve of capsaicin on the gastric transmucosal potential difference (GTPD) in healthy human subjects (Mózsik Gy. et al., 2005), when the different doses were directly intragastrically via endoscopic chanel. The GTPD measurements were carried out at 1, 2, 3, 4, and 5 minutes. The results are expressed at 5 min after capsaicin intragastric application. 


\section{Preventive effect of capsaicin on the decrease of GTPD caused by topical application of ethanol ( $5 \mathrm{~mL}, 30 \mathrm{v} / \mathrm{v} \%)$}

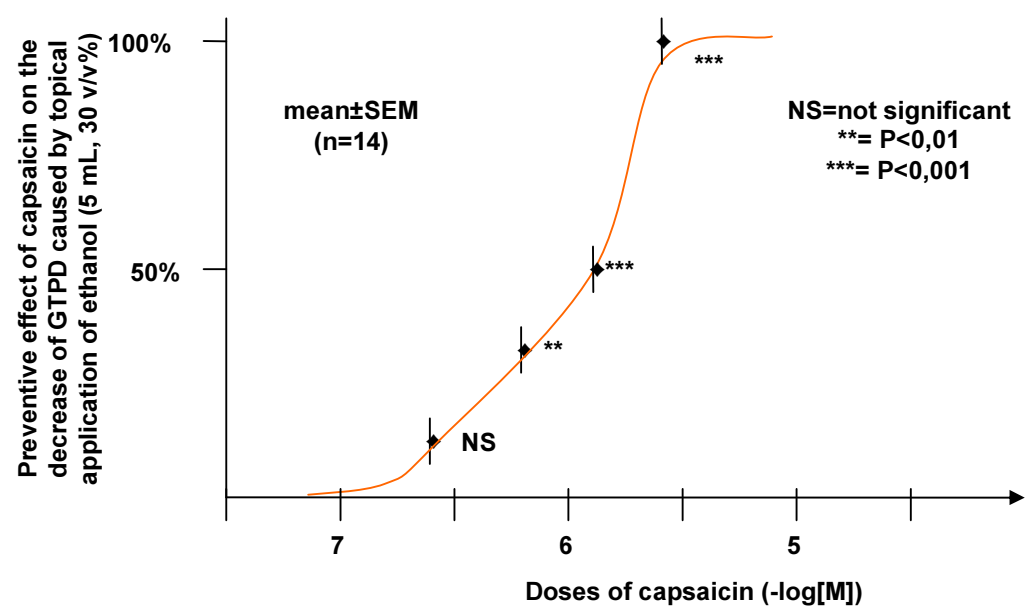

Figure 10. Dose-response curve of capsaicin on the gastric transmucosal potential difference after intragastric administration of ethanol in healthy human subjects (Mózsik Gy. et al., 2005). Intragastrically applied (via endoscopic chanel) ethanol ( $5 \mathrm{~mL}$ of $30 \mathrm{v} / \mathrm{v}$ ) decreased GTPD with $30 \mathrm{mV}$ which reversed with intragastric application of capsaicin at 1-2 min after capsaicin intragastric application.

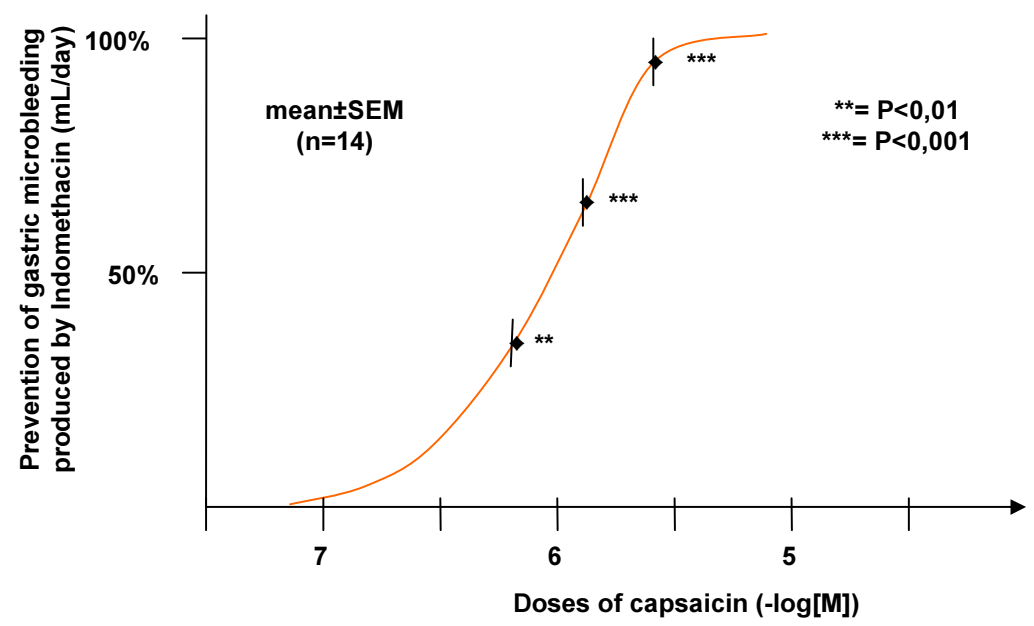

Figure 11. Dose-response curve of capsaicin on the Indomethacin $(3 \times 25+25 \mathrm{mg}$ given orally in pills) induced gastric microbleeding in healthy human subjects. The different doses 200, 400, $800 \mu \mathrm{g}$ capsaicin was intragastrically applied in gelatin capsule (Mózsik Gy., et al., 2005). 
4.2.8.1. Results of the comparative molecular-pharmacological studies of capsaicin, atropine,

Omeprazole, Famotidine, Ranitidine and Cimetidine on the gastric basal acid output (BAO) in human subjects

The affinity ( $\mathrm{pD}$ values) and intrinsic activity ( $\mathrm{pA}$ values) curves of capsaicin, atropine, Omeprazole, Famitidine, ranitidine and cimetidine (used in their physiological and human therapeutic doses) were determined in patients with gastrointestinal disorders, according to the method of Csáky (1969) (Figures 12 and 13, Table 8).

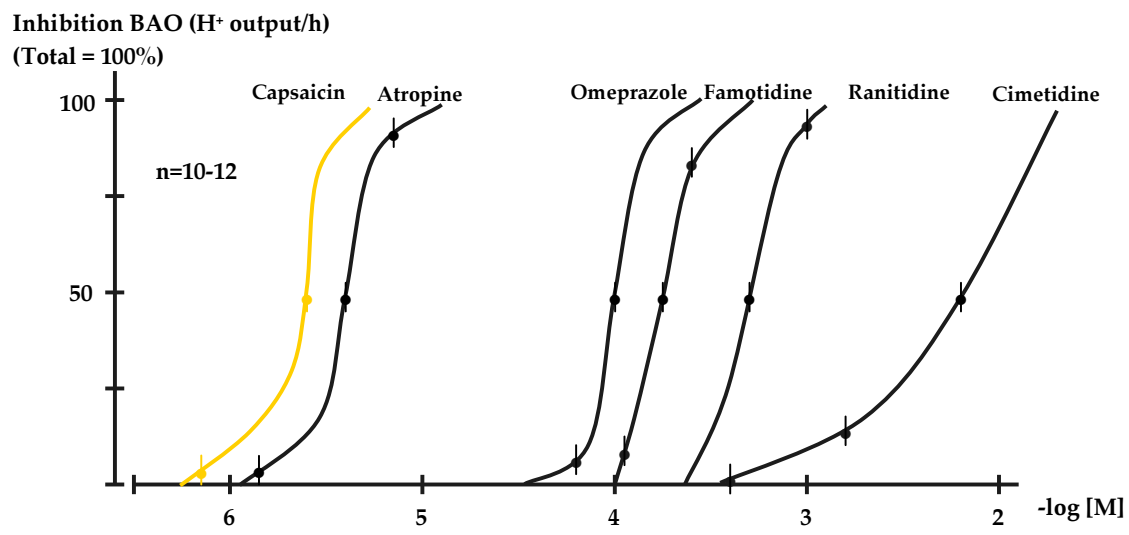

Figure 12. Affinity curves for the inhibitory actions of different drugs on the gastric basal acid output ( $\mathrm{H}^{+}$output/h) in healthy human subjects (Mózsik et al. 2007c). For further explanation see the cited paper.

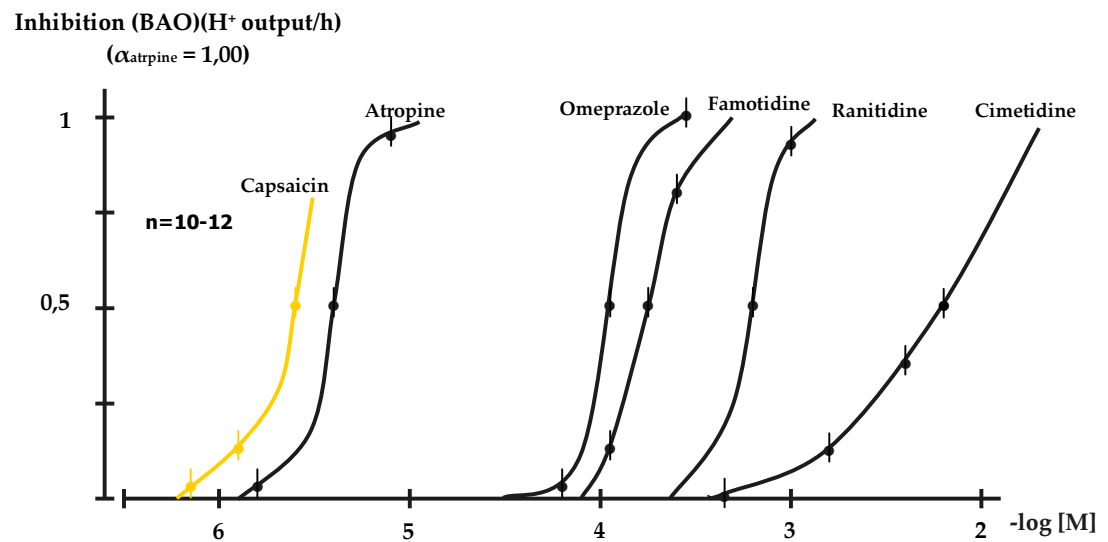

Figure 13. Intrinsic activity curves for the inhibitory effects of different drugs on the gastric basal acid output $\left(\mathrm{H}^{+}\right.$output/h) in healthy human subjects, which were expressed to action of atropine $(1,00)\left(a_{\text {atropine }}\right)$ (Mózsik et al. 2007c). For further explanation see the cited paper. 


\begin{tabular}{lllll}
\hline Compounds & $\mathbf{M} \mathbf{W} \mathbf{~}$ & $\mathbf{p D}_{\mathbf{2}}$ & Intrinsic activity & $\mathbf{p A}_{\mathbf{2}}$ \\
\hline Capsaicin & 305,4 & 5,88 & 0,76 & 5,87 \\
\hline Atropine & 289,38 & 5,40 & 1,00 & 5,40 \\
\hline Pirenzepine & 424,34 & 3,93 & 0,89 & 3,93 \\
\hline Cimetidine & 252,34 & 2,23 & 1,00 & 2,23 \\
\hline Ranitidine & 314,41 & 3,33 & 1,00 & 3,33 \\
\hline Famotidine & 337,43 & 3,77 & 1,00 & 3,77 \\
\hline Nizatidine & 331,47 & 3,34 & 1,00 & 3,34 \\
\hline Omeprazole & 345,42 & 3,97 & 1,00 & 3,97 \\
\hline Esomeprazole & 345,42 & 3,97 & 1,00 & 3,97 \\
\hline
\end{tabular}

Table 8. Summary of the affinity $\left(\mathrm{pD}_{2}\right)$ and intrinsic activity (expressed in value of $\left.\mathrm{a}_{\text {atropine }}=1,00\right)\left(\mathrm{pA}_{2}\right)$ values of capsaicin, atropine, Pirenzepine, cimetidine, ranitidine, famotidine, Omerazole and Esomeprazole on the gastric basal acid output (BAO) in healthy human subjects

\subsubsection{Side effects of aspirin and other nonsteroidal anti-inflammatory drugs (NSAIDs) in the gastrointestinal tract of patients}

The patients with myocardial infarction, thromboembolic episodes, stroke of central nervous system, cancers and persons who have to be under secondary preventional terapy (against reinfarction after myocardial infarction, prevention of thromboembolic episodes produced by atrial fibrillation, cancers, after different surgical intervention and immobilization) and in healthy subject treated with NSAIDs in order to prevent the development of colorectal cancers were randomized for further study.

The number of these groups of patients reaches to 50-60 per cent of total population in Hungary; however, an extremely big number of patients with these diseases is included in the different countries of the World.

The patients who underwent cardiac and other surgeries we have to treat permanently with aspirin (in dose around $100 \mathrm{mg} /$ day / person). This is a basic staind-point of the different consensus meetings of Europe and of the World (Mcgettigan and Henry, 2006; Expert Consensus Document on the Use of Antiplatelet Agents 2004; Todd, and Clissold, 1990).

The administration of aspirin is absolutely indicated from medical points of view in patients with mentioned disorders, accepting the opinion of cardiologists we have to emphasize that the aspirin very frequently produces harmful gastrointestinal bleeding (which is not accepted by gastroenterologists). Really, there is a great and principal question from the general medical practice (and of research), namely wheather all patients can be taken uniformally, in whom the medical science offers such medical treatment from the point of cardiology, which produces severe gastrointestinal disorders (bleedings, peptic ulcer). Consequently, there is a contradiction medically (and evidence-based proved) in standpoint of cardiologists and gastroenterologists (during the treatment only one patient, and as well as in the treatment of populations of patients mentioned above). 
Another big population of patients suffers from different degenerative joint diseases, trauma, acute and chronic pain producing states. These patients have to receive permanently treatment with NSAIDs. The NSAIDs are not gastrointestinal protective agents neither in healthy person and nor in patients with these diseases. The patients appearing at ambulance of Gastrointestinal Units are suffered from the drug-induced side effects in gastrointestinal tract

The number of patients with NSAIDs-induced gastrointestinal disorders (blood losing, bleeding, peptic ulcer) also represents a significant number of populations. Furthermore, these patients have to be treated permanenly by different NSAIDs.

The actions of NSAIDs are associated with the selective and non-selective inhibitory properties on cycloxygene system (emphasizing the key role of COX-1 and COX-2). Aspirin represents as a specific COX-1 inhibitor; meanwhile the other NSAIDs applied in the clinical practice represent the compounds acting as non selective COX-1 and COX-2 inhibitors. Recently, the specifically acting compounds, inhibiting COX-2 enzyme, have been produced, however, it was also observed that during the treatment an extremely big number of patients received myocardial infraction (Couzin, 2004a, 2004b; Lenzer, 2004; McGettigan, and Henry, 2006).

We have to emphasize clearly, that the small doses of capsaicin are able to prevent gastric mucosal bleeding in humans, which are able to inhibit both the COX-1 and COX-2 enzymes. This discovery opened a new pathway in the physiological and pharmacological regulation of different tissues (see later).

\subsection{Animal observations}

\subsubsection{Acute toxicology studies of capsaicin in animal experiments}

During acute toxicity study with capsaicin eight of 17 rats died in which capsaicin was administered in four increasing s.c. doses to a cumulative amount of $21.0-66.0 \mathrm{mg} / \mathrm{rat}$ (Cabanac et al., 1976). Additionally, Molnár (1965), Molnár and György (1967) reported that capsaicin administered i.v. at a dose higher than $10 \mu \mathrm{g} / \mathrm{kg}$ to cats caused a rapid fall in the mean arterial pressure which was followed by either a pressor phase or death.These results called our attention to methods of administration of capsaicin (intravenously or orally in animal observations.

The $\mathrm{LD}_{50}$ dose of capsaicin (given i.p.) was calculated as $7.65 \mathrm{mg} / \mathrm{kg}$ in adult female and male mice (please to note that capsaicin was not given in as pure agents, however as extracts) (Glinsukon et al., 1980). The toxicity of capsaicin present in the capsicum extract was approximately four fold higher than that of pure capsaicin given intraperitoneally to mice. Capsaicin had a sligtly $\mathrm{LD}_{50}$ in weanking female rats administred in propylene glycol of those observed when administered intraperitoneally in dimethylsulfoxyde (DMSO) $(P<0.05)$ (Glinsukon et al., 1980). Guinea pigs are the most susceptible species to capsaicin toxicity with $\mathrm{LD}_{50}$ of 1.10 $\mathrm{mg} / \mathrm{mg}$, whereas hamsters and rabbits are less susceptible (Glinsukon et al., 1980).

The main results of these toxicological observations were as follows: $\mathrm{LD}_{50}$ values were 7.65 $(5.28-11.09)$ for male and $6.50(4.33-9.75) \mathrm{mg} / \mathrm{kg}$ for female mice; $10.40(9.71-18.12) \mathrm{mg} / \mathrm{kg}$ 
for rats; $1.10(0.79-1,52) \mathrm{mg} / \mathrm{kg}$ for guinea pig; $>50 \mathrm{mg} / \mathrm{kg}$ for rabbits and $>120 \mathrm{mg} / \mathrm{kg}$ for hamsters (Glinsukon et al., 1980).

The relative lethality of capsaicin administered by various routes in the mouse:

- $0.56(0.36-0.87) \mathrm{mg} / \mathrm{kg}$ intravenously,

- $1.60(1.03-2.48) \mathrm{mg} / \mathrm{kg}$ intratracheally,

- $7.65(5.28-11.09) \mathrm{mg} / \mathrm{kg}$ intraperitoneally,

- $7.80(5.53$ - 10.99) $\mathrm{mg} / \mathrm{kg}$ subcutaneusly,

- 60 to $190 \mathrm{mg} / \mathrm{kg}$ intragastrically,

- $>218 \mathrm{mg} / \mathrm{kg}$ intrarectally and

- > $512 \mathrm{mg} / \mathrm{kg}$ dermally (Glinsukon et al., 1980).

At the autopsy, only hyperemia without hemorrhage was observed in the visceral organs and the muscular wall of the peritoneal cavity with a slight increase in the amount of peritoneal fluid in the rats treated intraperitotoenally treated with capsaicin. A similar observation was also found in mice treated intraperitoneally with capsaicin. Histopathologic changes seen in the gastric mucosa of mice treated intragastrically with capsaicin was desquamatic necrosis with increase mucus material (PAS's Schiff stain). Some of the chief and parietal cells showed an appearance of pale basophylic cytoplasm and vacuolization. No significant histopathological changes were observed in other organs.

The pattern of the electrocardiogram and heart rate did not change for $5 \mathrm{~min}$ after capsaicin adminsitration. Respiratory rates were slightly increased during the first min, whereas a small increase of the tidal volume was also observed. The tidal volume then decreased to 10 to 20 per cent of the control whithin 3-4 min and the respiration stopped. During this time, heart rate gradually decreased and electrocardiograph signals disappeared much later (in about 6-14 min). Mean arterial pressure was variable in the rats treated with capsaicin. At the beginning, capsaicin causes a transient hypotension and followed by hypertensive period. Mean arterial pressure gradually decreased along with the decrease in the tidal volume. Convulsions were not observed in these anesthetized with a lethal dose of capsaicin. This finding was confirnmed in mice anesthetized with sodium barbital and subsequently given a single lethal dose of capsaicin.

The $\mathrm{LD}_{50}$ values indicate a high susceptibility, in guinea pigs, rats and mice, whereas hamsters and rabbits are less susceptible to capsaicin. Capsaicin is a highly toxic compound when administered by all routes except gastric, rectal and dermal. Cabanac et al. (1976) published a report on the acute toxicity of capsaicin in which adult male rats were given four increasing subcutaneosly doses of capsaicin (cumulative amount of 21.0 to $66.0 \mathrm{mg} / \mathrm{kg}$ ).

The letality of capsaicin administered gastrically to the mouse is much more less than that of the intraperitoneal adminsitration route. The minimum lethal dose of capsaicin per $\mathrm{kg}$ was 100 $\mathrm{mg}$, which would be contained in $32.4 \mathrm{~g}$ dry weigth of fruits. For a $60 \mathrm{~kg}$ person, this toxic level 
would be comparable to the consumption of about $1.94 \mathrm{~kg}$ of dry weet of capsinum fruits, this prevents the over consumption of this spice (Molnar, 1965).

\subsubsection{Acute toxicity studies with pure trans-capsaicin derivated to dogs after intravenous administration}

The trans-geometric isomer of capsaicin, or trans-8-methyl-N-vanillyl-6-nonenamide, is the most abundant pungent molecule in chilli peppers and thus represents the most important ingredient in spicy foods. Although there are two geometric isomers of capsaicin (trans and cis), only trans-capsaicin occurs naturally (Cordell and Araujo, 1993). The capsaicin content of chilli peppers ranges from 0.1 to $1.0 \% \mathrm{w} / \mathrm{w}$ (Govindajarajan and Sathyanarayna, 1991). Furthermore, this food addidive has been widely used to evaluate the different physiological or pathological regulatory mechanisms in the human observation in the form of non-prescription (in USA) or prescribtion (in the Europe) topical analgetics, and self-defense products (e.g. pepper spray).

The trans-geometric isomer of capsaicin is a highly selective agonist for the transient receptor potential vanilloid receptor 1 (TRVP1 or also known as VR1 according to older nomenclature) (Caterina et al., 1997).

TRVP1 is a ligand-gated, non-selective, cation channel preferentially expressed in smalldiameter in primary afferent neurones (C-fibres and A $\delta$-fibres), especially nociceptive sensory nerves. TRPV1 responds to noxious stimuli including capsaicin, heat and extracellular acidification, and integrates simultaneous exposures to these stimuli (Tominaga et al., 1998). Based on the highly selective agonistic property of capsaicin toward TRVP1 receptors, drug products containing pure synthetic trans-capsaicin are under evaluation as topical and injectable therapies (Bley, 2004).

Formal studies of the toxicological potential of capsaicin in vivo began in 1935, when De Lille and Ramirez (1935) reported that administration of a capsaicin extract into dogs produces a fall in blood pressure accompanied by variable effect on the respiration, an increase is salivary secretion, and a relatively small increase in gastric secretion. The capsaicin can really be increased the buffering ("non-periatal component" of the gastric secretion, in association with the decrease of "parietal component" of gastric secretion by the application of pure capsaicin (Sigma, USA) in the human healthy subjects (Mózsik et al., 2007).

The capsaicin materials tested in the studies cited above were generally natural extracts and may not exhibit the same toxicological profile as pure synthetic trans-capsaicin. Although the extract content and nature of impurities in the test articles used in these studies are often not explicitly stated, a typical capsaicin extract is a mixture of trans-capsaicin (cis-capsaicin does not occur naturally) and other capsaicinoids (including capsaicin, nordyhydrocapsaicin, dihydrocapsaicin, homocapsaicin and homodihydrocapsaicin). Earlier Burk et al. (1986) clearly proved that there is no physiological difference of between the application of capsaicin or dihydrocapsaicin in animal experiments.

The actual percent of capsaicin and other capsaicinoids will vary depending on the peppers used and method of extraction. In fact, the United States Pharmacopoecia definies capsaicin 
as a product which contains $>55 \%$ capsaicin and in combination of capsaicin and dihydrocapsaicin to be $>\mathbf{7 5} \%$; total capsainoid content may be as little as $\mathbf{9 0} \%$ (United States Pharmacopoeica, 2005). Additionally, each extracts are expected to contain chemical entities other than vanilloid compounds.

Chanda et al. (2005) performed observation with pure trans-capsaicin in dogs. The objectives of their study were to evaluate the possible cardivascular and respiratory effects of the pure capsaicin, and to evaluate the potential of any target organ toxicity and that might occur as a result of introduction of the pure trans-capsaicin given into the systemic circulation in dogs.

These studies were carried out in approximatively 10-17 months old dogs weighed between 19 and $21.8 \mathrm{~kg}$ at the time of observations. Capsaicin for this study was dissolved in $10 \% \mathrm{w} / \mathrm{v}$ hydroxypropyl- $\beta$-cyclodextrin (Aldrich Chemical Gillingham, UK). Doses of capsaicin used in the main study were $0.03,0.1$ and $0.3 \mathrm{mg} / \mathrm{kg}$ given intravenously. The studies were carried out in acute observations and after two weeks of capsaicin (in doses of $0.03,0.1$ and 0.3 given intravenously) treatment as well. Different biochemical parameters (glucose, urea nitrogen, creatinine, total protein, albumin, albumin/globulin ratio, cholesterol, alanine aminotransferase, alkaline phosphatae, calcium, gamma glutamyltransferase, inorganic phosphorus, sodium, potassium, chloride, total bilirubin), hematological parameters (red blood count, hemoglobin, hematocrit, mean corpuscular volume, mean corpuscular hemoglobin, mean corpuscular hemoglobin concentration, platelet count, leucocyte count, different blood cell count, blood smear, prothombin time, activated partial thromboplastin time). Urine sapmles were tested for appearance/color, volume, specific gravidity, $\mathrm{pH}$, protein, glucose, ketones, bilirubin, blood, microscopic examination of urine sediments, and urobilinogen. At the necropsy, the macroscopic observations were recorded, the organs were weighed, and selected tissues were collected and preserved. Microscopic examinations were carried out from all the tissue samples.

The capsaicin concentration of the plasma samples was determined by high performance liquid chromatography (HPLC).

- All of these observations were carried out in acute and after two weeks capsaicin treatment.

- The studies reported were conducted according to the priciples of Good Laboratory Practice (GLP). The trans-capsaicin (CAS 404-86-4) used in both the studies decribed was manufactured under the Current Good Manufacturing Practice (CGMP) conditions. The two batches of trans-capsaicin used for the studies had $>99 \%$.

- The main results of these observations with trans-capsaicin in acute administration (before a chronic capsaicin treatment) are the followings:

\subsubsection{Acute effects on cardiovascular and respiratory parameters}

Administration of capsaicin (given in vehicle, 0.03 and $0.1 \mathrm{mg} / \mathrm{kg}$ ) had no detectable effect on the cardiac and respiratory systems. However, after administration of $0.3 \mathrm{mg} / \mathrm{kg}$ transcapsaicin elicited a rise in mean arterial blood pressure from a baseline of $96 \pm 7$ to $138 \pm 21 \mathrm{mmHg}$ whithin 2 min of starting the infusion. This effect peaked at $146 \pm 17 \mathrm{mmHg}$ at the end of infusion 
(15 min). The administration of the vehicle, capsaicin from $0.03 \mathrm{mg} / \mathrm{kg}$ to $0.1 \mathrm{mg} / \mathrm{kg}$ had no measurable effectc on arterial blood pressure, change in force of ventricular contraction $(\mathrm{dP} /$ $\left.\mathrm{dt}_{\max }\right)$, heart rate ECG waveform, and femoral blood flow. However, administration of $0.3 \mathrm{mg} /$ $\mathrm{kg}$ capsaicin elicited a rise in mean arterial blood pressure from a base line of $96 \pm 7$ to $138 \pm 21$ $\mathrm{mmHg}$ within $2 \mathrm{~min}$ of starting the infusion. The hypertensive effect was accompanied by increases in heart rate (from $71 \pm 3$ at baseline to $119 \pm 25 \mathrm{bpm}$ ), $\mathrm{dP} / \mathrm{dt}_{\max }$ (from $4050 \pm 91$ at baseline to $6679 \pm 1027 \mathrm{mmHg} / \mathrm{s}$ ) and femoral blood flow (from $117 \pm 27$ at baseline to $174 \pm 34 \mathrm{~mL} / \mathrm{min}$ ). These changes were statistically significant $(P)$ in comparison with the results obtained after application of vehicle, 0.03 or $0.1 \mathrm{mg} / \mathrm{kg}$ capsaicin injection.

The change in heart rate was also associated with decreases in the RR and QT intervals of the ECG. However the corrected QT intervals (both $\mathrm{QT}_{\mathrm{CF}}$ and $\mathrm{QT}_{\mathrm{CB}}$ ) were unchanged.

The administration of the vehicle elicited a decrease in deep of respiration. This was reflected as decreases at 30 min post-infusion in tidal volume (TV) (from $143 \pm 19$ to $118 \pm 20 \mathrm{~mL}$ ), peak inspiratory flow (PIF) (from $253 \pm 12$ to $198 \pm 10 \mathrm{mLs}$ ) and peak expiratory flow (PEF) (from $297 \pm 47$ to $246 \pm 24 \mathrm{~mL} / \mathrm{s}$ ). The rate of respiration was unaffected. After administration of 0.03 and $0.1 \mathrm{mg} / \mathrm{kg}$ trans-capsaicin did not elicit any further changes in respiration. After administration of high $(0.3 \mathrm{mg} / \mathrm{kg}$ ) dose of trans-capsaicin (given intravenously) elicited increases in PIF, PEF and TV. The increase of in PIF and PEF following $0.3 \mathrm{mg} / \mathrm{kg}$ trans-capsaicin is significantly different from the decrease in these parameters following the vehicle treatment $(P<0.05)$. However, these increases were transients, lasting for only 5 to $10 \mathrm{~min}$ following the infusion.

\subsubsection{Plasma levels of capsaicin}

No detectable levels of capsaicin were found $5 \mathrm{~min}$ after administration of $0.05 \mathrm{mg} / \mathrm{kg}$ capsaicin. Following the administration of the intermediate dose $(0.1 \mathrm{mg} / \mathrm{kg}$ i.v. $)$, two and four dogs showed detectable levels of capsacinin (in ranges approximatively 17 and $11 \mathrm{ng} / \mathrm{mL}$ ). The high dose $(0.3 \mathrm{mg} / \mathrm{kg})$ produced an increase in the plasma levels in all dogs (ranging 32.2 to $65.6 \mathrm{ng} / \mathrm{mL}$, mean of $47.9 \pm 6.4 \mathrm{ng} / \mathrm{mL}$ ).

\subsubsection{Results of subacute toxicology of capsaicin in dogs}

\subsubsection{Two weeks treatment with trans-capsaicin}

All dogs survived until scheduled termination on Day 15. Only capsaicin solution-related clinical sign observed during the study was vacualization during dosing, which was noted in all dogs.

In general, the observation was noted more frequently in male dogs than in female dogs. Clear nasal discharge was seen across all groups, and the daily incidence was slighly higher in male than in females given $0.3 \mathrm{mg} / \mathrm{kg}$ trans-capsaicin (intravenously), althought the daily incidence in males given 0.1 or $0.3 \mathrm{mg} / \mathrm{kg} /$ day of trans-capsaicin and in females given $0.3 \mathrm{mg} / \mathrm{kg} /$ day was slightly higher than controls. Slight tremors (head, limbs and/or body) were seen across all groups during this study. The majority of these observations were noted during the study. 
As the study progressed, dogs given $0.3 \mathrm{mg} / \mathrm{kg}$ trans-capsaicin demonstrated an apparent tolerance to the general anesthetic and analgesic, as indicated by a general vacualization during the dosing period.

There were no statistically significant differences in the body weights and food consumption values, among the groups of dogs treated with capsaicin chronically. The body weights, however, were slightly lower (in about $7 \%$ ) in males treated with $0.3 \mathrm{mg} / \mathrm{kg}$ trans-capsaicin for 14 days. Over the duration of the study, males lost approximately $0.4 \mathrm{~kg}$, whereas the control gained $0.1 \mathrm{~kg}$. Through this value statistically was not significant, the food consumption of males and females given $0.3 \mathrm{mg} / \mathrm{kg} /$ day was sligthly lower than that of controls (approximately 11 and 12 per cent, respectively).

\subsubsection{Clinical chemistry and hematology}

The only the difference considered related to test article was minimally higher ALT for males and females given $0.3 \mathrm{mg} / \mathrm{kg} /$ day trans-capsaicin intravenously after 14-day treatment. Other statistically significant differences for clinical chemistry test results were considered incidental, because they did not exhibit dose relatioship and they were present before the initiation of treatment. In hematology, WBC was statistically significant $(\mathrm{P}<0.05)$ lower in female dogs in the $0.3 \mathrm{mg} / \mathrm{kg} /$ day treated group. A few animals, including controls, had notably high neutrophil counts, which were likely secondary to inflammated lesions at injection sites.

\subsubsection{Organ weights, macroscopic and microscopic observations}

There were no capsaicin solution-related organ weight changes, macroscopic or microscopic observations.

The statistically significant differences with respect to controls of prostate, brain and adrenal weight values were considered incidental because there were no correlating macroscopic and microscopic findings. Thrombosis, due to administration of vehicle, was noted at the intravenous injection sites in all groups. Other lesions observed with the thrombosis induced inflammmation, fibrosis, edema and hemorrhage.

\subsubsection{Pharmacokinetic data after 14-day treatment with trans-capsaicin in dogs}

After intravenous administration, peak of the plasma concentration $\left(C_{\max }\right)$ was obtained in all cases immediately at the end of infusion. Capsaicin was rapidly eliminated and measurable values were only obtained immediately after the end of infusion $(0.25 \mathrm{~h})$ in $0.03,0.1$ and 0.3 $\mathrm{mg} / \mathrm{kg} /$ day dose groups. In the $0.3 \mathrm{mg} / \mathrm{kg} /$ day group, measurable values were obtained at 0.5 $\mathrm{h}$ in all dogs on Day 1, but they were very close to the limit of quantitation (10 ng/mL). On Day 15 , only one dog still had a measurable value at this timepoint.

Females generally had higher or similar mean $\mathrm{C}_{\max }$ values compared to males, but the largest difference did not exceed $44 \%$. The increases in mean $\mathrm{C}_{\max }$ for males and females were throughly proportional to the increase of dose level from 0.03 to $0.3 \mathrm{mg} / \mathrm{kg} / \mathrm{day}$. 


\subsubsection{Absorption and metabolism of oral application of the capsaicinoids in animal experiments}

Due to the increasing experimental use and planned drug production in humans of capsaicin in a very wide field of medical research and medical treatment, we have to know correct facts on the absorption, metabolism and excretion of capsaicinoids.

It is known that capsaicin given directly into the stomach of rats has only minimal excitatory effects on immediate blood pressure responses (Lippe et al., 1989) in contrast to intravascular or subcutaneous administration (Donnerer and Lembeck, 1983). On the other hand it has been shown that capsaicin disappears from the intestinal lumen whithin a rather short time (Kawada et al., 1984) and should therefore reach the circulation.

Since biotransformed products of capsaicin are difficult to detect, the use of $\left[{ }^{3} \mathrm{H}\right]$-labelled dihydrocapsaicin $\left(\left[{ }^{3} \mathrm{H}\right]-\mathrm{DHC}\right)$ allowed us to determine the percentage of unchanged compound in the total ectracted radioactivity. Dihydrocapsaicin (DHC) has been shown to display pharmacodynamic and pharmacokinetic properties compared with those of capsaicin (Burk et al., 1982; Kawada et al., 1984).

$\left[{ }^{3} \mathrm{H}\right]$-dihydrocapsacin $\left(\left[{ }^{3} \mathrm{H}\right]-\mathrm{DHC}\right)$ and unlabelled capsaicin were readily absorbed from the gastrointestinal tract but were almost completely metabolized before reaching the general circulation. A certain degree of biotransformation already took place in the intestinal lumen. Unchanged compounds (identified by chromatography) were present in the portal vein blood. These seem to be result of a saturable absorption and degradation process in the gastrointestinal tract and a very effective metabolism limit of the liver (Donnerer et al., 1990).

Less than $5 \%$ of the total amount of extracted radioactivity consisted of unchanged $\left[{ }^{3} \mathrm{H}\right]-\mathrm{DHC}$ in truck blood and brain 15 min after gastrointestinal application. On the other hand, approximatively $50 \%$ unchanged $\left[{ }^{3} \mathrm{H}\right]-\mathrm{DHC}$ was detected in these tissues in $3 \mathrm{~min}$ after intravenously application or $90 \mathrm{~min}$ after subcutaneously application of capsaicinoids (Donnerer et al., 1990). Dihydrocapsaicin (DHC) or $\left[{ }^{3} \mathrm{H}\right]-\mathrm{DHC}$ were metabolized when incubated in vitro with liver tissue but not with brain tissue (Donnerer et al., 1990). The metabolic product(s) did not show capsaicin-like biological activity (Donnerer et al., 1990). These results clearly indicate that the rapid hepatic metabolization limits systemic pharmacological effects of enterally absorbed capsaicin in rats (Donnerer et al., 1990)

\subsubsection{Toxicological studies with pure trans-capsaicin derivated to dogs after intravenous administration in acute and after subacute experimental circumstances}

\subsubsection{Chemistry of capsaicin}

Trans-capsaicin, or trans-8-methyl-N-vanillyl-6-nonenamide, is the most abundant pungent molecule in chilli peppers and thus represents the most important ingredient in spicy foods. Although there are two geometric isomers of capsaicin, only the trans-isomer occurs in natural sources (Cordell and Araujo, 1993). The capsaicin content of chilli peppers ranges from 0.1 to $1.0 \% \mathrm{w} / \mathrm{w}$ (Govindajarajan and Sathyanarayna, 1991). Furthermore, this food addidive has been widely used to evaluate the different physiological or pathological regulatory mecha- 
nisms in the human observation in the form of non-prescription (in the USA) or prescribtion (in Europe) topical analgetics, and self-defense products (e.g. pepper spray).

The trans-geometric isomer of capsaicin is a highly selective agonist for the transient receptor potential vanilloid receptor 1 (TRVP1 or as known VR1 according to older nomenclature) (Caterina et al., 1997).

TRVP1 is a ligand-gated, non-selective, cation channel preferentially expressed in small-

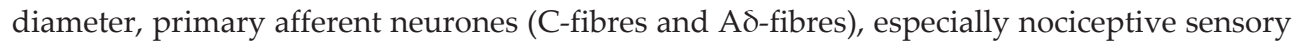
nerves. TRPV1 responds to noxious stimuli including capsaicin, heat and extracellular acidification, and integrates simultaneous exposures to these stimuli (Tominaga et al., 1998). Based on the highly selective agonistic property of capsaicin toward TRVP1 receptors, drug products containing pure synthetic trans-capsaicin are under evaluation as topical and injectable therapies (Bley, 2004).

Formal studies of the toxicological potential of capsaicin in vivo began in 1935, when De Lille and Ramirez (1935) reported that administration of a capsaicin extract into dogs produced a fall in blood pressure accompanied by variable effect on the respiration, an increase is salivary secretion, and a relatively small increase in gastric secretion. The capsaicin really can increase the buffering ("non-perietal component" of the gastric secretion, in association with the decrease of "parietal component" of gastric secretion by the application of pure capsaicin (Sigma, USA) in human healthy subjects (Mózsik et al., 2007a).

The capsaicin materials tested in the studies cited above were either natural extracts or racemic mixtures, and may not exhibit the same toxicological profile as pure trans-capsaicin. Although the extract content and nature of impurities in the test articles used in these studies are often not explicitly stated, a typical capsaicin extract is a mixture of trans-capsaicin (cis-capsaicin does not occur naturally) and other capsaicinoids (including capsaicin, nordyhydrocapsaicin, dihydrocapsaicin, homocapsaicin and homodihydrocapsaicin). Earlier Burk et al. (1986) clearly proved that there is no physiological difference between the applications of capsaicin and dihydrocapsaicin in animal experiments).

\subsubsection{Observations in acute experiments in the dogs}

The actual percent of capsaicin and other capsaicinoids will vary depending on the peppers used and method of extraction: In fact, the United States Pharmacopocia definies capsaicin as a product which contains $>55 \%$ capsaicin and in combination of capsaicin and dihydrocapsaicin to be $>75 \%$; total capsainoid content may be as little as $90 \%$ (United States Pharmacopoeica, 2005). Additionally, per extracts are expected to contain chemical entities other than vanilloid compounds.

Chanda et al. (2005) did observation with pure trans-capsaicin in dogs. The objectives of this study were to evaluate the possible cardiovascular and respiratory effects of the pure capsaicin, and to evaluate the potential of any target organ toxicity and that might occur as a result of introduction of the pure trans-capsaicin given into the systemic circulation in dogs. 
These studies were carried out in dogs, approximatively 10-17 months old and weighed between 19 and $21.8 \mathrm{~kg}$ at the time of observations. Capsaicin for this study was dissolved in $10 \% \mathrm{w} / \mathrm{v}$ hydroxypropyl- $\beta$-cyclodextrin (Aldrich Chemical, Gillingham, UK). Doses of capsaicin used in the main study were $0.03,0.1$ and $0.3 \mathrm{mg} / \mathrm{kg}$ given intravenously. The studies were carried out in acute observations, and after two weeks capsaicin (in doses of 0.03, 0,1 0nd $0,3 \mathrm{mg} / \mathrm{kg}$ given intravenously) treatment. Different biochemical parameters (glucose,urea nitrogen, creatinine, total protein, albumin, albumin / globulin ratio, cholesterol, alanine aminotransferase, alkaline phosphatae, calcium, gamma glutamyltransferase, inorganic phosphorus, sodium, potassium, chloride, total bilirubin), hematological parameters (red blood count, hemoglobin, hematocrit, mean corpuscular volume, mean corpuscular hemoglobin, mean corpuscular hemoglobin concentration, platelet count, leucocyte count, differenrt blood cell count, blood smear, prothombin time, activated partial thromboplastin time). Urine samples were tested for appearance/color parameters, volume, specific gravidity, $\mathrm{pH}$, protein, glucose, ketones, bilirubin, blood, microscopic examination of urine sediments, and urobilinogen. At the necropsy, the macroscopic observations were recorded, the organs were weighted, and selected tissues were colected ans presenved. Microscopic examinations were carried out from all the tissue samples.

The capsaicin concentration of the plasma samples was determined by high performance liquid chromatography (HPLC).

All of these observations were carried out in acute and after two weeks capsaicin treatment.

The studies reported were conducted according to the priciples of Good Laboratory Practice (GLP). The trans-capsaicin (CAS 404-86-4) used in both the studies decribed was manufactured under the Current Good Manufacturing Practice (CGMP) conditions. The two batches of transcapsaicin used for the studies had $>99 \%$.

The main results of these observations with trans-capsaicin in acute administration (before a chronic capsaicin treatments) are as follows:

Administration of capsaicin (given in vehicle, 0.03 and $0.1 \mathrm{mg} / \mathrm{kg}$ ) had no detectable effect on the cardiac and respiratory systems. However, after administration of $0.3 \mathrm{mg} / \mathrm{kg}$ transcapsaicin elicited a rise in mean arterial blood pressure from a baseline of $96 \pm 7$ to $138 \pm 21 \mathrm{~mm}$ $\mathrm{Hg}$ whithin $2 \mathrm{~min}$ of starting the infusion. This effect peaked at $146 \pm 17 \mathrm{mmHg}$ at the end of infusion (15 $\mathrm{min}$ ). The hypertensive effect was accompanied by increases in heart rate (from $71 \pm 3$ at baseline to $119 \pm 25 \mathrm{bpm}$ ) left ventricular pressure and derivitave $\left(\mathrm{dP}^{\mathrm{d}} \mathrm{dt}_{\max }\right)$ (from $4050 \pm 91$ at baseline to $6679 \pm 1079 \mathrm{mmHgs}$ ), and femoral blood flow (from $117 \pm 27$ at baseline to $174 \pm 35 \mathrm{~mL} / \mathrm{min}$ ). These changes were statistically significant in comparison with the results obtained after vehicle, 0.03 or $0.1 \mathrm{mg} / \mathrm{kg}$ capsaicin injection

Administration of the vehicle elicited a decrease in deep of respiration. This was reflected as decreases at $30 \mathrm{~min}$ post-infusion in tidal volume (TV) (from $143 \pm 19$ to $118 \pm 20 \mathrm{~mL}$ ), peak inspiratory flow (PIF) (from $253 \pm 12$ to $198 \pm 10 \mathrm{mLs}$ ) and peak expiratory flow (PEF) (from $297 \pm 47$ to $246 \pm 24 \mathrm{~mL} / \mathrm{s}$ ). The rate of respiration was unaffected. After administration of 0.0 .3 and $0.1 \mathrm{mg} / \mathrm{kg}$ trans-capsaicin did not elicit any further changes in respiration. After administration of high $(0.3 \mathrm{mg} / \mathrm{kg})$ dose of trans-capsaicin (given intravenously) elicited increases in 
PIF, PEF and TV. The increase of in PIF and PEF folloowing $0.3 \mathrm{mg} / \mathrm{kg}$ trans-capsaicin is significantly different from the decrease in these parameters following the vehicle treatment $(P<0.05)$. However, these increases were transients, lasting only 5 to $10 \mathrm{~min}$ after the end of infusion.

No detectable levels of capsaicin were found in the plasma level at 5 min after administration of $0.05 \mathrm{mg} / \mathrm{kg}$ capsaicin. Following the administration of the intermediate dose $(0.1 \mathrm{mg} / \mathrm{kg}$ i.v. $)$, two and four dogs showed detectable levels of capsacinin (in ranges approximatively 17 and $11 \mathrm{ng} / \mathrm{mL})$. The high dose $(0.3 \mathrm{mg} / \mathrm{kg})$ produced an increase in the plasma levels in all dogs (ranging 32.2 to $65.6 \mathrm{ng} / \mathrm{mL}$, mean of $47.9 \pm 6.4 \mathrm{ng} / \mathrm{mL}$ ).

\subsubsection{Results of the subacute and chronic toxicology of capsaicinoid in dogs}

All dogs survived until the scheduled termination on day 15 . Only capsaicin solution-related clinical sign observed during the study was vacualization during dosing, which was noted in all dogs.

In general, the observation was noted more frequently in male dogs than in female dogs. Clear nasal discharge was seen across all groups, and the daily incidence was slighly higher in male than in females given $0.3 \mathrm{mg} / \mathrm{kg}$ trans-capsaicin (intravenously), althought the daily incidence in males given 0.1 or $0.3 \mathrm{mg} / \mathrm{kg} /$ day of trans-capsaicin and in females $0.3 \mathrm{mg} / \mathrm{kg} / \mathrm{day}$ was slightly higher than controls. Slight tremors (head,l imbs, and/or body) were seen across all groups during this study. The majority of these observations were noted during the study.

As the study progressed, dogs given $0.3 \mathrm{mg} / \mathrm{kg}$ trans-capsaicin demonstrated an apparent tolerance to the general anesthetic and analgesic, as indicated by a general vacualization during the dosing period.

There were no statistically significant differences in the body weights and food consumption values, among the groups of dogs treated chronically. The body weights, however, were slightly lower (in about $7 \%$ ) in males treated with $0.3 \mathrm{mg} / \mathrm{kg}$ trans-capsaicin for 14 days. Over the duration of the study, males in this group lost approximately $0.4 \mathrm{~kg}$, whereas the control gained $0.1 \mathrm{~kg}$. Altough it was statistically not significant, the food consumption of males and females given $0.3 \mathrm{mg} / \mathrm{kg} /$ day was sligthly lower than that of controls (approximately 11 and 12 per cent, respectively) (Changa et al., 2005).

\subsubsection{Clinical chemistry and hematology}

The results of the clinical chemistry [aminotransferase (ALT), aspartate aminotransferase (AST) and alkaline phosphatase], and hematology (RBC, count of $\mathrm{MCV}, \mathrm{MCV}$, platelet count,WBC) tests from days -1 and 15 only are measured. There were few statistically significant or otherwise notable differences for clinical chemistry test results between the control and treated animals.

The only the difference considered related to test article was minimally higher ALT for males and females given $0.3 \mathrm{mg} / \mathrm{kg} /$ day trans-capsaicin intravenously after 14 days treatment. Other significant differences for clinical chemistry test results were considered incidental because 
they exhibited no dose relationship or were present before initiation of the treatment. In hematology, female dogs in the $0.3 \mathrm{mg} / \mathrm{kg} /$ day treated group the WBC was statistically significant $(P<0.05)$ lower. A few animals, including controls, had notably high neutrophil counts, which were like secondary to inflammated lesions at injection sites.

\subsubsection{Organ weights, macroscopic and microscopic observations}

There were no capsaicin solution-related organ weight changes in macroscopic or microscopic observations.

The statistically significant differences with respect to controls of prostate, brain and adrenal weight values were considered incidental because there were no correlating macroscopic and microscopic findings.

Thrombosis, due to administration of vehicle, was noted at the intravenous injection sites in all groups. Other lesions observed with the thrombosis induced inflammation, fibrosis, edema and hemorrhage.

\subsubsection{Pharmacokinetic data after 14 days treatment with trans-capsaicin in dogs}

After intravenous administration, peak plasma concentration $\left(\mathrm{C}_{\max }\right)$ was attained in all cases immediately after the end of infusion. Capsaicin was rapidly eliminated and measurable values were only obtained immediately after the end of infusion ( 0.25 hour) in $0.03,0.1$ and $0.3 \mathrm{mg} / \mathrm{kg} /$ day dosage groups. In the $0.3 \mathrm{mg} / \mathrm{kg} /$ day group, measurable values were obtained out to 0.5 hour in all dogs on day 1 , but they were very close to the limit of quantitation (10 $\mathrm{ng} / \mathrm{mL}$ ). On day 15 , only one dog still had a measurable value at this timepoint.

Females generally had higher or similar mean $C_{\max }$ values compared to males, but the largest difference did not exceed $44 \%$. The increases in mean $\mathrm{C}_{\max }$ for males and females were throughly proportional to the increase of dose level from 0.03 to $0.3 \mathrm{mg} / \mathrm{kg} /$ day. Mean $\mathrm{C}_{\max }$ values for males increased 1: 3.3: 10-fold on Day 1 and 1: 2.9: 9.2 fold on Day 14. These results clearly indicate that no acumulation exists to capsaicin after multiple dosing by this route of its administration (Chanda et al., 2005).

\subsubsection{Summary and conclusions of the administration of trans-capsaicin in its acute and subacute experiments in dogs (on dependence of different doses of trans-capsaicin)}

In the acute study, surface lead II ECG was monitored to determine the QTc intevals and the duration of cardiac repolarization. However, there were no observable changes in the $\mathrm{QT}_{\mathrm{CF}}$ (Fridericia's correlation $\mathrm{QT}_{\mathrm{CF}}, \mathrm{QT}_{\mathrm{CF}}=\mathrm{QT} / \sqrt{ } \sqrt{ }(\mathrm{RR}$ intervall)). Such a change would have been theoretically possible, as capsaicin has been reported to block voltage-activated potassium channels in rat ventricular myocytes (Castle, 1992). Because many drugs are able to block voltage-activated channels actuallly shorten the duration of cardiac action potentials, there are limited correlations between the potassium channel blocking activity and QT interval prolongation (Martin et al., 2004). The lack of measurable effects on the cardiac action potential make it likely that the hemodynamic effects of capsaicin measured during the acute study with 0.3 
$\mathrm{mg} / \mathrm{kg} / \mathrm{kay}$ capsaicin due to agonistic activity on TRVP1 receptors. This is probably due to the reported potency of capsaicin being severalfold higher than for either calcium and potassium ion channels (Castle, 1992); Cheng et al., 2003). Thus, it is likely that capsaicin receptors expressed on pericardiac sensory nerves induced the transient increases in the heart rate observed during the acute dosing study, in the course of performing their role to sense cardiac ischemia (Pan and Chem, 2004). For longer administration of capsaicin, it is possible that the putative antihypertensive actions of capsaicin result from either prolonged desensitation of pericardiac sensory fibres, or including the release of vasoactive peptides (CGRP and SP) from the perivascular C-fibres, or activating and endogenous system with counterbalances hypertension caused by sodium salt loading (Vaishnava and Wang, 2003).

Other than cardiac effects, capsaicin has also been studied in animals for other possible target organ toxicity. Almost all of these studies used pepper plant extracts, which are likely to display varying degrees of capsaicinoids content and possible diverse impurity profiles. These impurities may be the contributing factor in some of toxicities observed. Chanda et al. (2004) observed that pure capsaicin displays a different genotocity profil than that described in some previous literature. Additionally, in contrast to the high dose levels used in toxicological studies, human exposure to dietary capsaicinoids (a mixture of capsaicin, dihydrocapsaicin, nordihydrocapsaicin, homocapsaicin and homodihydrocapsaicin) in the USA and in European countries is about $1.5 \mathrm{mg} /$ day, which translates into $0.025 \mathrm{mg} /$ day/ day dose (Chanda et al., 2005).

When capsaicin was dissolved in diethylene gycol monoethyl eher and Dulbecco's phosphate-buffered saline and administered intravenous infusion for14 days (in a $15 \mathrm{~min}$ time period to anesthetized dogs), the vehicle itself caused marked vascular irritation at the administration sites. There were no deaths, no test article-related organ weight changes, and no macroscopic and microscopic observations. The only test article-related clinical sign observed in the study was vacualization by the dogs treated with $0.3 \mathrm{mg} / \mathrm{kg} /$ day dose. The only test article-related clinical pathology finding was maximal higher in ALT for male and female dogs receiving $0.3 \mathrm{mg} / \mathrm{kg} /$ day capsaicin. This may indicate the liver as a possible target organ when capsaicin is delivered at high doses directly into the systemic circulation. When the capsaicin was given in dose of $0.1 \mathrm{mg} / \mathrm{kg} /$ day for 14 days, capsaicin was rapidly eliminated in dogs.

Capsaicin dissolved in dimethyl sulfoxide (DMSO) has been studied by Glinsukon et al. (1980) for determination of $\mathrm{LD}_{50}$ values using several administration routes in mice. The authors also determined the $\mathrm{LD}_{50}$ values of capsaicin for one administration route (intraperitoneal) in different species. The oreder of sensitivity ( $\mathrm{LD}_{50}$ values) for species, from at least to most, by the intraperitoneal route using DMSO as the delivery vehicle was repoted to be: hamsters $(>120 \mathrm{mg} / \mathrm{kg})$, rabbit $(>50 \mathrm{mg} / \mathrm{kg})$, rat $(9.5 \mathrm{mg} / \mathrm{kg})$, mouse $(6.5$ to $7.65 \mathrm{mg} / \mathrm{kg})$ and guinea pig $(1.1 \mathrm{mg} / \mathrm{kg})$. This study and those described above were mixed with respect to gender. Electrocardiograms (ECGs), mean arterial presure and resporiratory rates were also measured by Glinsukon et al. (1980) in anesthetized rats after treatment with a lethal intraperitoneal dose of capsaicin. Saito and Yamamoto (1996) reported the oral $\mathrm{LD}_{50}$ values for capsaicin extract for both genders in mice and rats from experiment, where propylene 
glycol was used as vehicle. They did not found any significance in the $\mathrm{LD}_{50}$ values in both genders. The major toxic signs in mice and rats included salivation, straggering gait, bradypnoe and cyanosis. Tremor, clonic convulsion, dyspnoe and lateral or prone position were observed, and then the animals died in time period of 4 to $26 \mathrm{~min}$ after oral dosing by gavage.

The cause of death was proposed to be due to hypotension and respiratory paralysis in rats and mice although the authors noted that the pathophsiology of these deaths was not clearly understood.

In a study with Monsereenusorn (1983), $50 \mathrm{mg} / \mathrm{kg}$ capsaicin was given orally by gavage to rats for up to 60 days. The effects of capsaicin on body weight, rectal temperature, food and water consumption, hematological parameters, plasma biochemistry, urine concentration and dilution tests were evaluated at 10,20,30, 40, 50 and 60 days. The major finding was decreased body weight gain starting at 40 days, despite an increase in food cosumption. Minor changes in the clinical chemistry (reduced plasma urea nitrogen, glucose, phospholipids, triglycerides, total cholesterol, free fatty acids, glutaminic piruvic transaminase and alkalic phosphatase) were noted after one month treatment, however, these differences were not biologically significant,

The results of observations done by Chanda et al. (2005) indicate that trans-capsaicin given into the sytemic circulation, induces transient increases in heart rate and blood pressure without the alterations in cardiac repolarization.

\subsubsection{Metabolism of capsaicin}

Early studies by Lee and Kumar (1980) showed that phenobarbital-induced rat liver microsomes converted the capsaicin and dihydrocapsaicin to corresponsing catechol metabolites, $\mathrm{N}$-(4,5-dihydroxyl-3-methoxybenzyl)-acetamides via hydroxylation on the vanillyl moiety. This finding was further confirmed by Miller et al. (1983) who demonstrated the covalent binding of $[3 \mathrm{H}]$-dihydrocapsaicin to hepatic microsomal proteins following in vitro incubation or administration to rats. Based on these results, it has been postulated that capsaicin is activated by the liver mixed-function oxidase system to an electrophilic intermediate, most likely a ring epoxide, capable of covalently interactiving with nucleophilic sites of hepatic protein (Figure 14).

This irreversible interaction of capsaicin with liver microsomal protein may account for its impact binding of capsaicin observed in spinal cord or brain, and it was concluded that capsaicin-induced neuropathy in rodents might be mediated by mechanisms other than covalent intercation (Miller et al.,1983). The alkyl side chain of capsaicin is also considered to be susceptible to enzymatic oxidation. Thus, when capsaicin was incubated with NADPH and the liver S9 fraction from phenobarbital pretreated rats, it was hydroxylated at the terminal carbon of the side chain (Surh et al., 1995). 

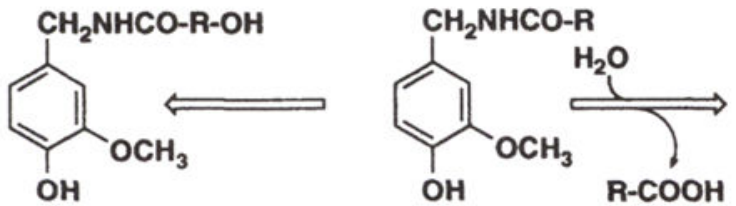<smiles>COc1cc(CN)ccc1O</smiles>

CAPSAICIN

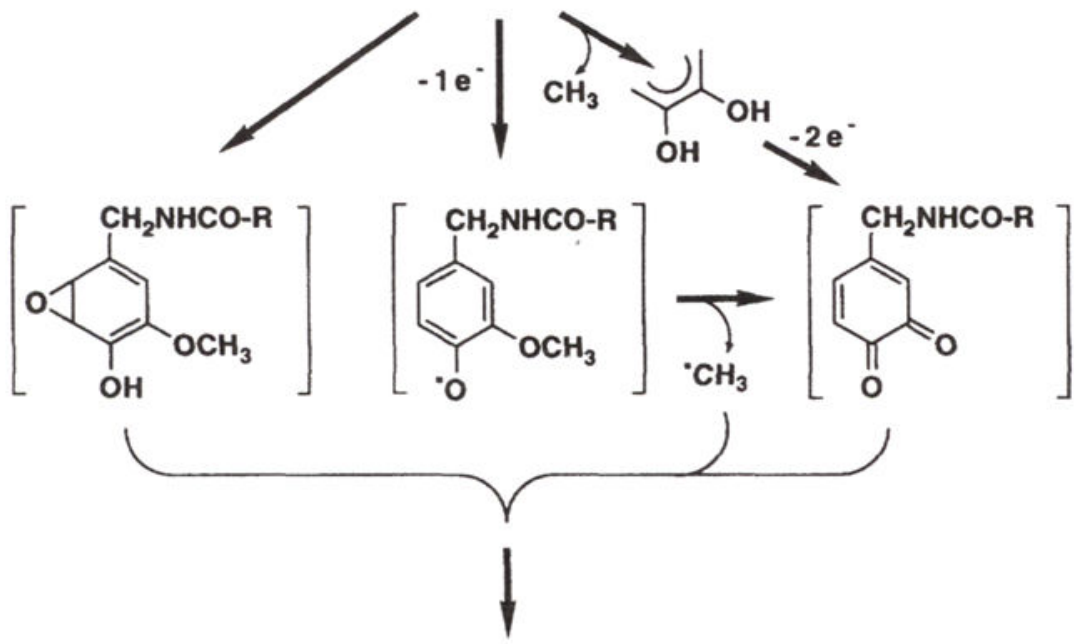

COVALENT INTERACTION WITH CRITICAL CELLULAR NUCLEOPHILES

(e.g., PROTEINS, DNA, RNA...)

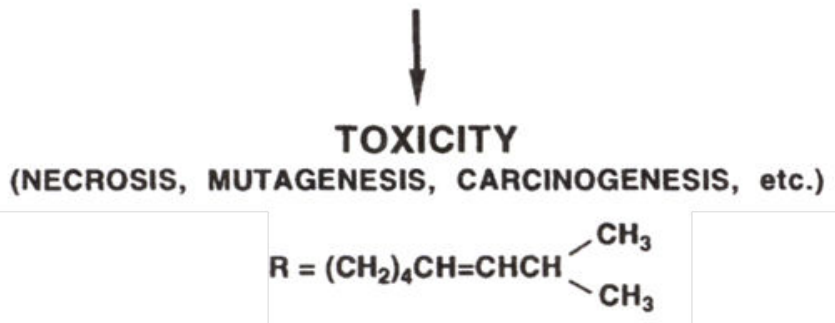

Figure 14. Metabolism of natural capsaicin in the experimental animals. After Surh and Lee, 1995 with modification.

\subsubsection{The potential routes of metabolism in capsaicin}

\subsection{Enzymatic oxidatic metabolism of capsaicin}

One-electron oxidation of capsaicin has been investigated by means of electrochemical, enzymatic and chemical procedures (Lawson and Gannett, 1989; Boersch et al., 1991). Lawson and Gannett (1989) reported that incubation of capsaicin with microsomes or non-enzymatic reaction with potassium ferricyanide resulted in the formation of a dimer, 5,5'-bis-capsaicin. 
A phenoxy radical was proposed to be involved in the mutagenesis by capsaicin. Formation of phenoxy radical has often been observed with certain plant phenolics (Newmark, 1984; 1987), which plays a critical role of in lignin biosynthesis in the process of wood formation (Freudenberg, 1960). Formation of dimetric tyrosine by oxidation of tyrosine with horseradish peroxidase-catalized coupling reaction has been proposed as a mechanism for the dimerization of two diiodotyrosyl residues in thyroglobin to form the thyroid hormone, thyroxine (Taurog et al., 1994). Boersch et al. (1991) have demonstrated that incubation of capsaicin with peroxidase and hydrogen peroxide produced a flouescent dimer analogue similar to that previously reported by Lawson and Gannett (1989). The formation of this flourescent oxidation product was also observed by chemical or electrochemical oxidation of capsaicin (Lawson and Gunnett, 1989). Gunnett et al. (1990) has shown that the liver cytochrome P450 2E1 (CYP2E1) activity is responsible for conversion to the reactive phenoxy radical which, in turn, dimerizes or covalently binds to CYP2E1, thereby interacting the enzyme (Figure 14).

\subsection{Non-oxidative metabolism of capsaicin}

Cell-free extracts of various tissues of rats contained enzyme activity for hydrolyzing capsaicin or its dihydro analoque at the acid-amide bond to produce vanillylamine and the corresponding fatty acyl moieties (Kawada and Iwai, 1985; Tawada et al., 1992; Oi et al., 1992) (Table 1). The highest enzyme activity was found in the liver followed by such extrahepatic tissues as kidney, lung and small intestine (Kawada and Iwai, 1985). It is of oral administration of capsaicin (Oi et al., 1992). The splitting of the side chain of dihydrocapsaicin also occurred in vivo (Kawada and Iwai, 1985), which is considered to be rate-limiting step in the overall metabolism of this compound. Hydrolysis of the amide linkage of capsaicinoids will thus lead to the formation of vanillylamine as a common metabolite regardless of the fatty acid type in their side chain. Indeed, the systhemic vanilloid, olvanil [N-(3-methoxy-4-hydroxybenzyl)oleamide] having longer side chain than capsaicin, has been found to be susceptible to hydrolysis of the amide bond as determined in various metabolic model systems including cell-free extracts of liver and intestine, isolated hepatocytes, enterocytes, and perfused isolated intestine, and also in whole animal studies (Wehmeyer et al., 1990). Oxydative deamination of the resulting vanillylamine produces the aromatic alcohol for excretion as a free form or a glucoronic conjugate (Kawada and Iwai, 1985; Wehmeyer et al., 1990). Capsaicin hydrolyzing enzymes have been purified from the rat hepatic microsomes (Park and Lee, 1994), and identified as previously known isoenzymes of carbylesterase based on such biochemical and biophysical parameters as $\mathrm{Mr}$, pI value, $\mathrm{pH}$-dependency, mode of inhibition and subcellular toxicity. The enzymes are likely to be present either free in the lumen of endoplasmatic reticulum or loosely bound to the terminal surface of the membrane (Park and Lee, 1994). Capsaicinoids administered to rats intragastrically were readily absorbed from the gastrointestinal tract, and further metabolized to a great extent in the liver before reaching the general circulation (Park and Lee, 1994). As a result, gastrointestinally absorbed capsaicinoids are expected to reach the central nervous system or other extrahepatic organs almost exclusively as degradation products (Donnerer et al., 1990). 


\subsubsection{Role of metabolic activation in capsaicin-induced toxicity}

There is no clear-cut mechanism which can solely explain the toxicity exerted by capsaicin. Bioactivation to an electrophylic intermediate with subsequent covalent modification of critical cellular macromolecules such as DNA, RNA and proteins has been thought to play a role in cell death (Miller et al., 1983; Anonymous, 1986), fueling interest in towards the role of these observed metabolic processes. Based on the results of previous metabolic studies, the following activation pathways can be postulated which may account for the capsaicin-induced cellular damage (Figure 15):

a. cytochrome P450-catalyzed epoxidation of the vanillyl moiety to produce an arene oxide;

b. one-electron oxidation of the hydroxyl group to form a phenoxy radical;

c. O-demethylation at the aromatic ring and subsequent oxidation of the resulting catechol to the semiquinone and quinone derivates.

The possible involvement of an electrophilic epoxide by others (Miller et al., 1983), but the presumed oxirane epoxidation of capsaicin is expected to occur since the formation of arene oxide. Nonetheless, ring epoxidation of capsaicin is expected to occur since the formation of arene oxide intermediates is a best general phenomenon in the monooxigenase-catalized metabolism of aromatic compounds. The best evidence for the epoxidation of capsaicin might be the actual isolation of the presumed arene oxide, but the chemical reactivity of such species precludes its direct isolation from incubation mixtures or from the biological fluids or tissues of treated animals. The advances in the development of novel mild oxidating agents such as dimethyl dioxirane have made it possible to prepare the extremely reactive epoxides of certain chemical carcinogens including alflatoxin B1-8,9 oxide (Baertschi et al., 1988). A similar synthetic approach could be applied to synthesis of the benzoepoxide derivate of capsaicin for testing its biological activity as well as chemical reactivity. The covalent binding of tritiumlabeled capsaicin to hepatic microsomal protein was significantly inhibited by reduced glutathione, which implies the formation of a reactive intermediate (epoxide) during metabolism of capsaicin (Miller et al., 1983). Since glutathione is relatively nonspecific in terms of interacting with reactive intermediates including not only oxiranes but also of capsaicin does not necessarily suggest the aforementioned arene oxide as a sole electrophilic epoxide hydrolase being more sensitive approach in exploring the possible involvement of an epoxi metabolite in the toxification processes induced by capsaicin.

The intermediacy of the phenoxy radical of capsaicin has been investigated by using the electrochemical or chemical methods (Lawson and Gannett, 1989; Boiesch et al., 1991; Gannett et al., 1990). Furthermore, horseradish peroxidase plus the phenoxy radical intermediate (Boesch et al., 1991; Gunnett et al., 1990). Likewise, hepatic microsomal cytochrom P450 (particularly CYP2E1) might generate the same reactive radical species that is capable of attacking the nucleophilic sites of the enzyme or the target cell protein (Gunnett et al., 1990) leading to the loss of catalytic activity in other crutial biological functions.

A quinone type intermediate also represents a potential ultimate electrophilic metabolite of capsaicin. The formation of such intermediate could proceed via O-demethylation of the 3- 


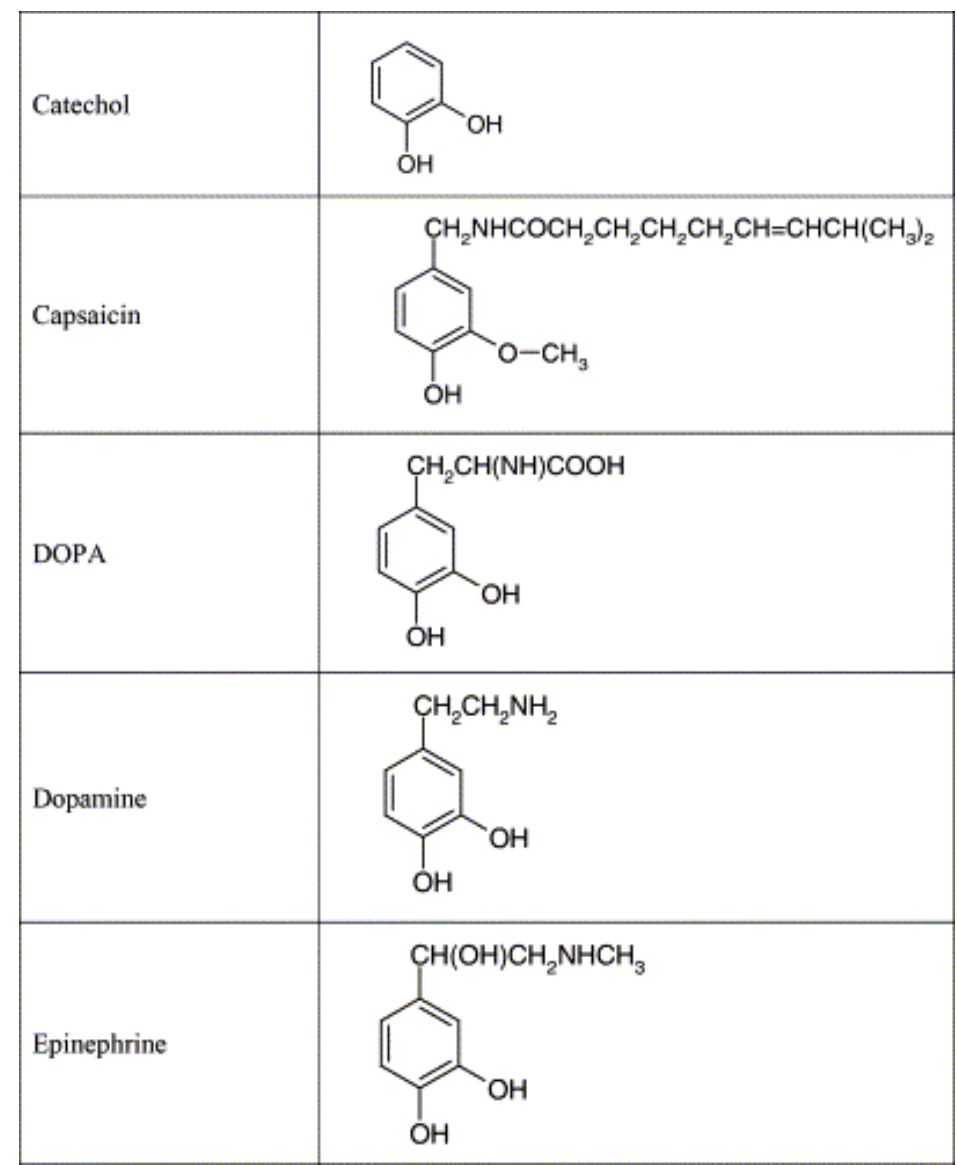

Figure 15. Structures of selected compounds containing cathecol moieties, including capsaicin. (Chanda et al., 2004)

methoxy group on the vanillyl moiety with concomittant oxidation to the semiquinone and ortho-quinone derivates. The same of ortho-quinone metabolite could be generally through Odemethylation of the aforementioned phenoxy radical intermediate of capsaicin (Table 1). This process will also lead to the formation of an extremely reactive $\mathrm{CH}_{3}$ radical, which is wel known to alkylate cellular nucleic acids and proteins. The above mentioned reactions are likely to occur in presence of microsomal O-demethylase activity and relatively high reactivity of the catechols. The antineoplastic agent, etoposide, for instance, has been known to exert its cytotoxic effect by enzymic O-demethylation of one of its methoxy groups to the ortho-quinone derivative capable for binding covalently to cellular macromolecules (Haim et al., 1987a; 1987b; Mans et al., 1991; Relling et al., 1992; 1994). Similarly, initial ring epoxidation of capsaicin and subsequent NIH shift resulting benzoepoxide derivate would generate a catechol intermediate without O-demethylation 


\subsubsection{Effects of capsaicin on xenobiotic metabolism and chemically induced mutagenesis and carcinogenesis}

Capsaicin has been suggested to exert such chemopreventive effects through modulation of metabolism of carcinogens and their interactions with tareget cell DNA. It has been reported that capsaicin displays a dose-related inhibition of the activity of rat epithelial aryl hydrocarbon hydroxylase (Modly et al., 1986), a marker enzyme for metabolism of polycyclic aromatic hydrocarbons such as benzo $(\alpha)$ pyrene. Furthermore, capsaicin suppressed the metabolism and covalent DNA binding of benzo $(\alpha)$ pyrene and human as well as murine keratocytes (Modly et al., 1986). The results of different studies suggested the interaction of capsaicinoids with microsomal mixed-function oxidases.Thus, the pretreatment of rats with subcutaneous dosages of capsaicin resulted in pronounced prolongation of fenobarbital or hexobarbital sleeping time (Miller et al., 1983; Surh et al., 1995; Rauf et al., 1985). Capsaicin also competitively inhibited the ethylmorphine demethylase activity in rat liver microsomes and produced a type I spectral change (Miller et al., 1980). Oral administration of capsaicin $(50 \mathrm{mg} / \mathrm{kg})$ together with $10 \%$ ethanol in drinking materials resulted much greater xenobitic metabolizing enzyme activity, than that induced by ethanol alone (Iwama et al., 1989). Capsaicin pretreatment also induced 4-hydroxylation of biphenyl in the rat liver (Rauf et al., 1985).

Yagi has reported that capsaicin and dihydrocapsaicin repress the energy-transducing NADHquinone oxidoreductase activity (Yagi, 1990). This finding confirms an earlier observation by investigations of inhibitory effects of capsaicin on rat hepatic mitochondial energy metabolism through supression of energy flow from NADH to coenzyme Q (Chudapongse and Jathanasoot, 1881). Capsaicin was shown to inhibit calmodulin-mediated oxidative burst in rat macrophages as determined by attenuetion of $\mathrm{Ca}^{2+}$ ionophore-triggered production of superoxide anion and hydrogen peroxide (Savitha et al., 1990). Joe and Lokesh (1994) have also shown that capsaicin can strongly block the generation of reactive oxygen species in rat peritoneal macrophages in vitro. Capsaicin fed to animals was also inhibitory onto these macrophages (Joe and Lokesh, 1994). Pretreatment of rats with capsaicin $(1.68 \mathrm{mg} / \mathrm{kg}$, intraperitoeally) for three consequeutive days resulted in enhancement of activities of pulmonary antioxidant enzymes such as superoxide dismutase, catalase and peroxidase while long-term treatment caused an opposite effect on the latter two enzymes (De and Ghosh, 1989). Since reactive oxygen species are known to play an important role in phorbol-12-myristate-13acetate (PMA)-mediated tumor promotion as well as in inflammation (Kensler and Trush, 1989), it would be worth determining whether capsaicin with potential anti-inflammatory activity (Joe and Lokesh, 1994; Flynn and Rafferty, 1986) could act as an anti-tumor promoter. It is noteworthy that the inhibition of prostaglandin synthesis by curcumin, the product of tumeric, correlates well with its protective activity against curcumin by inactivate xanthine dehydrogenase/oxigenase, which may account for its anti-promotional activity.

Capsaicinoids have also been found to retain inhibitory effects on liver microsomal CYP2E1 activity (Gannett el al., 1990; Lee et al., 1994; Shlyankevich et al., 1995). Capsaicin inhibits the metabolism and mutagenicity (Guengerich et al., 19991; Koop, 1992; Espinosa-Aguirre et al., 1993), which is known to be activated by CYP2E1. The vinyl carbamate-induced CYP2E1mediated mutagenicity and tumorigenicity has been showns to be reduced by capsaicin (Lee 
et al., 1995). The initiation of papillomas in mouse skin by benzo $(\alpha)$ pyrene was also significantly reduced by topical application of capsaicin prior to the carcinogen (Lee et al., 1995). This finding is in agreement with in vitro inhibition of rodent epidermal arylhydrocarbon hydroxylase activity by capsaicin as previously reported by Moody et al. (1986). Chilli extracts have been found to modulate the mutagenic activity of particulate organic matter in urban air samples (Espinosa-Aquuirre et al., 1993).

In a series of observations, it has been observed that capsaicin has a protective effect on the metabolism, DNA binding, and/or mutagenicity of some carcinogens [aflatoxin Bl, tobaccospecific nitrosoamine and 4-(methylnitroso)-1-(3-pyridyl)-1-butanone)]. These findings suggest that capsaicin might act as a chemoprotective agent by modulating the activities of microsomal mixed-function oxidases which play key roles in metabolic activation as well as detoxication of a wide array of chemical carcinogens and mutagens (Surh and Lee, 1995).

\subsubsection{Hepatoprotection of capsaicin in rats}

Recently Abdel Salam et al. observed in details the dose dependent hepatoprotective effect of orally given capsacin ( $2 \mathrm{~mL} / \mathrm{kg}$ followed by $1 \mathrm{~mL} / \mathrm{kg}$ after one week) in rats treated with carbon tetrachloride $\left(\mathrm{CCl}_{4}\right)$ (2006). Capsaicin at three dose levels $(10,100$ and $1000 \mu \mathrm{g} / \mathrm{kg})$ or silymarin $\left(22 \mathrm{mg} / \mathrm{kg}\right.$ ) was administered orally for 10 days, starting at the time of administration of $\mathrm{CCl}_{4}$. The daily administration of capsaicin conferred significant protection against the hepatotoxic effect of $\mathrm{CCl}_{4}$ in rats. It decreased the increases of of serum alanine aminotransferase (ALT) and aspartate aminotransferase (AST) and also prevented the development of histological hepatic necrosis caused by $\mathrm{CCl}_{4}$ as determined 10 days after drug administration. Thus, compared with $\mathrm{CCl}_{4}$ control group, serum ALT decreased by 39.3, 59.3 and $71.1 \%$, while AST decreased by 14.3, 21.5 and $23.3 \%$, after the capsaicin administration (given it in doses mentioned above), respectively. Serum bilirubin was decreased by 10 and $100 \mu \mathrm{g} / \mathrm{kg}$ (46.4 and $66.5 \%$ reduction, respectively), but an increased bilirubin and ALP was observed after the highest dose of capsaicin. Meanwhile, silymarin reduced serum ALT by $65.3 \%$, AST by $18.9 \%$, ALP by $22 \%$ and bilirubin by $13.4 \%$, compared to controls. Serum proteins were significantly increased by 16.9 to $22.9 \%$ after treatment with capsaicin compared, whilst marked increased in serum glucose by $66.9 \%$ was observed after highest dose of capsaicin compared with vehicletreated group.

Quantitative analysis of the area of damage by image analysis technique showed a reduced area of damage from $13.6 \%$ to $7.5,4.3$ and $2.8 \%$ by application of capsaicin (used in the above mentioned doses) respectively (Abdel-Salam et al., 2006). Haematoxylin-eosin staining indicated markedly less hepatic necrosis in rats treated with capsaicin or silymarin. Histochemical alterations such as decreased nuclear DNA, cell glycogen and proteins contents caused by $\mathrm{CCl}_{4}$ in hepatocytes were prevented by capsaicin as well as by silymarin.

It has been concluded from these results that orally applied capsaicin exerts beneficial effects on liver histopathologic changes and enzymatic release caused by $\mathrm{CCl}_{4}$ in rats, but high doses are likely to result cholestasis (Abdel Salam et al., 2006). 


\subsubsection{Genotoxicity studies of capsaicin or trans-capsaicin}

Published information on the potential genotoxicty of capsaicin is inconsistent, both positive and negative effects have been found in classic genetic toxicology assays (Surh and Lee, 1995; Azazan and Blevins, 1995).

Eight bacterial point mutation tests (including Ames assays) were performed from 1981 to 1995 on capsaicin of carying origins, using variuos strains of S.typhimurium. Variopus forms of S9 activation were provided seven of the eight assays. Four of these tests resulted in a positive responses and four resulted in a negative response. Point mutation tests in Chinese hamster V79 cells were conducted twice, resulting in positive and one negative response. The in vivo micronucleus test was conducted once in mice and it was positive (Nagabhushan and Bhide, 1985). Data from one micronucleus and sister chromatid exchange study in human lymphocytes was interpreted to show that capsaicin is genotoxic (Marques et al., 2002). Capsaicin was also reported to induce DNA strand breaks in human neuroblastoma cells (Richeus et al., 1999).

The most of these studies were carried out with natural extracts, and these may not exhibit the same toxicological profile with pure capsaicin.

Recently different studies were carried out to evaluate the genotoxic potential of transcapsaicin using different genotoxic assays used by international regulatory agencies to evaluate drug product safety (Chanda et al., 2004). These included the Ames assay, mouse lymphoma cell mutations assay, mouse in vivo bone marrow micronucleus assay and chromosomal aberration assay in human peripheral blood lymphocytes (HPBL). All studies were conducted accoring to the Organization of Economic Cooperation and Development (OECD) principles Good Laboratory Practice (GLP)

\subsubsection{Ames assay}

Ames assay described in the paper of Chanda et al. (2004) was based on the method described by Ames et al. (1975). The thimidi kinase (TK) heterozygote system was described by Clive et al. (1972), in which tk+tk-is mutated to tk-tk-. It's measured on the L5178Y mouse lymphoma cell line established by Fischer (1958).

In this assay, cell deficient $\mathrm{tk}+/$ tk-to tk-tk-are resistant to cytotoxic effects of pyrimidine analoque triflurothymidine (TFT). Thymidin kinase proficient cells are sensitive to TFT, which causes the inhibtion of cellular metabolism and stops further cell division. Thus, the mutant cells are able to proliferate in the presence of TFT, whereas normal cells containing thymidine kinase are not (Moore et al., 2002).

Salmonella typhimurium TA 1535, TA 1537, TA 98 and TA 100 were used. The assays were performed in presence and absence of S9, using the direct method and preincubation method. Capsaicin did not induce mutagenic activity in any of bacterial strains.

\subsubsection{Mouse lymphoma cell mutation assay}

The tk+/tk-3.7.2C heterozygote of L5178Y mouse lymphoma cells were used in these studies (details of the methods used, see in paper of Chanda et al., 2004). 
Both assays in presence of S9 mix gave weak mutagenic responses. Both assays contained at least two treatment groups of capsaicin that tested sinificant (for log mutant fraction) at the level of $5 \%$. Both assays showed linear trend (of mutant fraction with concentration) that was significant at $P<0.001$. The increases in the mutant fraction obtained were small, the largest was 190 mutants per million above the control value obtained at near the maximum acceptable level of toxicity ( $12 \%$ relative total growth).

In the absence of S9 mix, the 4 hour exposure assay gave a very weak mutagenic response, while the 24 hour assay gave no significant responses at dose levels resulting acceptable level of genotoxicity, throughout the test linear trend was significant $(P=0.022)$.

Colony size distribution patterns were difficult to assess due to the very small sample sizes. The same situation occurs for vehicle control groups, which show a high level of variation between the experiments, and within experiments. The numbers of mutant colonies assessed in the capsaicin treatments resulting significant increases were found very low.

\subsubsection{Mouse in vivo micronucleus assay}

For details of this methodology, see the paper of Chanda et al. (2004). At least 2000 polychromatic erythrocytes were scored for frequency of micronucleated cells. The numbers of mironucleated normochromic erythrocytes (NCE), which were observed within the same microscope fields, were similarly recorded. The PCE/NCE ratio was assessed by scoring a total of at least 500 PCE+NCE. All assessment was performed on coded slides.

In preliminary toxicity tests the maximum tolerated dose of capsaicin was determined to be around $800 \mathrm{mg} / \mathrm{kg}$ per day in males and around $200 \mathrm{mg} / \mathrm{kg}$ per day in females. Three groups of male mice were dosed with 200, 400 and $800 \mathrm{mg} / \mathrm{kg}$ per day and one group of females was dosed with $200 \mathrm{mg} / \mathrm{kg}$ per day of capsaicin at 0 and 24 hours. Five mice per sex from each test material dose groups were selected to provide the normal assessment base. Current vehicle and positive groups were included.

Treatment-related animal deaths and clinical signs were observed in the middle and high dose level groups. One death occurred in the male vehicle control group, immediately after dosing and was considered to be as a result of a dosing error.

The frequencies of micronucleated polychromatic erythrocytes (MN-PCE) in the capsaicintreated groups were $0.04,0.10,0.09 \%$ (males) and $0.05 \%$ (females). All of these frequencies were within the historical control range for negative responses $(0.01-0.23 \%$ for a group of five mice). The frequencies of MN-PCE in the concurrent vehicle control groups were $0.07 \%$ (males) and $0.11 \%$ (females), whereas the MN-PCE frequency in the positive control group was $1.57 \%$, demonstrating the sensitivity of the test system.

\subsubsection{Chromosomal aberration in human peripheral blood lymphocytes (HPBL)}

Human venous blood from healthy, adult donors (nonsmokers without any history of radiotherapy, chemotherapy or drug usage and lacking current viral infections) was used. The whole blood cultures were initiated in $15 \mathrm{ml}$ centrifuge tubes by adding $0.6 \mathrm{~mL}$ of fresh heparinized blood and the final volume of culture medium and test arcticle was $10 \mathrm{~mL}$. 
Cultures were incubated with loose caps at $37 \pm 2{ }^{\circ} \mathrm{C}$ in a humidified atmosphere of $5 \pm 1.5{ }^{\circ} \mathrm{C}$ in air. The medium was RPMI 1640 supplemented with HEPES buffer ( $25 \mathrm{mM}$ ), about $20 \%$ heatactivated fetal bovine serum (FBS), penicillin (100 U/mL), streptomycin $100 \mu \mathrm{g} / \mathrm{mL}$, Lglutamine $(2 \mathrm{mM})$ and $2 \%$ phytohemagglutinin-M (PGA-M). Negative (untreated controls) and vehicle controls (cultures treated with $10 \mu \mathrm{L}$ of DMS0/mL) were used. The positive control agents used in the assays were mitomycin-C (MMMC) for the nonactivation series and $\mathrm{CP}$ (cyclophosphamide) in the metabolic activation series.

The in vitro metabolic activation system (Maron and Ames, 1983) consisted of liver postmitochondrial fraction (S9) and an energy-producing system (NADP at $1.5 \mathrm{mg} / \mathrm{mL}(1.8 \mathrm{mM})$ and isocitric acid at $2.7 \mathrm{mg} / \mathrm{mL}(10.5 \mathrm{mM})$. S9 was prepared 5 days after a single dose of 500 $\mathrm{mg} / \mathrm{kg}$ of Aroclor $^{\circledR} 1254$.

Two trials were conducted. In the initial trial, cultures were treated for about 3 hours with and without S9 and harvested about 22 hours after initiation of treatment. In the second trial, cultures were treated for about 22 hours without S9 and about 3 hours with S9 and harvested about 22 hours after initiation of treatment. This harvested time corresponds to 1.5 times a cell cycle time (Galloway et al., 2004). The time of cell cycle is approximatively $15 \mathrm{~h}$ after the lymphocytes was included to divide by the addition of PHA-M. At harvest, cells were swollen by $75 \mathrm{mM} \mathrm{KCl}$ hypotonic solution and fixed with absolute methanol-glacial acetic acid (3:1 v/ v). Cells were selected from each duplicate culture were analyzed for the different types of chromosomal aberrations (Evans, 1962; Evans, 1976). Mitotic index was evaluated from the negative control, vehicle control and a range of test article concentrations and this was used for measurement of toxicity and selection of doses for analysis. Percent of polyploidy and endoreduplication were also analyzed. For control of bias, all slides were coded prior to analysis and read blind.

In the first trial, 6.78, 9.69, 13.8, 19.8, 28. 2, 40.4, 57.6, 82.4, 118, 168, 240, 343, 490, 700 and 1000 $\mu \mathrm{g} / \mathrm{mL}$ of capsaicin were evaluated with and without metabolic activation by S9. The highest concentration was limited due to the presence of a precipitate dosing. In the first trial, a precipitate was observed at $>240 \mu \mathrm{g} / \mathrm{mL}$, and hemolysis was observed prior to washing and harvesting of these cultures. Only dead cells were present on slides prepared from cultures treated with $>343 \mu \mathrm{g} / \mathrm{mL}$ due to excessive toxicity (Chanda et al., 2004). Chromosomal aberrations were analyzed from the cultures treated with $82.4,118,168$ and $240 \mu \mathrm{g} / \mathrm{mL}$. The high concentration had $>50 \%$ reduction in mitotic index. No increase in structural or numerical chromosomal aberrations was observed (Chanda et al., 2004).

Based on the results from the initial assay, the second trial was conducted at concentrations of $6.15,12.3,24.6,49.2,98.4,154,192,240$ and $320 \mu \mathrm{g} / \mathrm{mL}$ without metabolic activation and 49.2, $98.4,123,154,192,240$ and $320 \mu \mathrm{g} / \mathrm{mL}$ with metabolic activation. Treatment periods were for about 22 and about $3 \mathrm{~h}$ without and with metabolic activation, respectively, and the cultures were harvested at $22 \mathrm{~h}$ from the initiation of treatment. In the assay without metabolic activation, a precipitate was observed in cultures treated with $>92 \mu \mathrm{g} / \mathrm{mL}$ and hemolysis was found prior to harvest of the cultures treated with $>240 \mathrm{~g} / \mathrm{mL}$. Only dead cells were present on slides prepared from cultures treated with $>192 \mu \mathrm{g} / \mathrm{mL}$, due to excessive toxicity. Severe toxicity was observed also in case of $98.4 \mu \mathrm{g} / \mathrm{mL}$ dose ( $92 \%$ reduction in mitotic index). Chromosomal 
aberrations were analyzed from the cultures treated with $24.6,49.2$ and $123 \mu \mathrm{g} / \mathrm{mL}$. Due to toxicity, $<100$ metaphases were available for analysis in the duplicate cultures treated with 123 $\mu \mathrm{g} / \mathrm{mL}$ dose. The highest tested concentration $(240 \mu \mathrm{g} / \mathrm{mL})$ had $>50 \%$ reduction on mitotix index. No increase in structural or numerical chromosomal aberrations was observed.

In the assay with metabolic activation, a precipitate was observed after dosing at $>192 \mu \mathrm{g} / \mathrm{mL}$ and hemolysis was observed prior to harvest of the cultures treated with $320 \mu \mathrm{g} / \mathrm{mL}$. Since the cultures treated with $240 \mu \mathrm{g} / \mathrm{mL}$ had excessive toxicity (100\% reduction in mitotic index) and the slides prepared from cultures treated with $192 \mu \mathrm{g} / \mathrm{mL}$ were selected as the highest concentration for analysis. Chromosomal aberrations were analyzed from the cultures treated with 98.4, 123, 154 and $192 \mu \mathrm{g} / \mathrm{mL}$. No increase in structural or numerical chromosomal aberrations was observed (Chanda et al., 2004).

Although there are a number of publications focussing on genotoxic potential of capsaicin or spicy pepper extracts, the test substances used in these studies were various according to source, purity and impurity profile. Consequently, there has not been any systemic observation of the genotoxic potential of trans-capsaicin.

The majority of the studies found in the literature (Marques et al., 2002; Richeux et al., 1999; Ames et al., 1975; Fischer, 1972) used the Ames assay. There was one study using either micronucleus assay, the sister chromatoid exchange assay or the assay investigating DNA strand breaks in human neuroblastoma cells (Surh and Lee, 1995; Azizan and Blevins,1995; Nagabhushan and Bhide, 1985; Marques et al., 2002; Ames et al., 1999). None of the studies were conducted using systematically pure capsaicin preparations, and it is very important to be able to attribute the results of capsaicin alone, not the impurities.Chanda et al. (2004) applied synthetic capsaicin alone (the purity $>99 \%$ ) to perform to genotoxicity studies.

In the Ames assay with pure synthetic capsaicin, no increase in mutation frequency was observed in any assays, with and without S9. It was concluded from these studies that the capsaicin is not genotoxic in the bacterial assay, with and without metabolic activation (at the highest concentrations that could be tested).

Capsaicin was found to be weakly mutagenic in mouse lymphoma L5178Y cells, in presence of S9, when it was dissolved in DMSO at concentrations that extended into the toxic range. The lowest positive concentration in the presence of S9 in any individual test was $12 \mu \mathrm{g} / \mathrm{mL}$. Limited evidence of very weak activity was also noted in the absence of S9.The lowest positive concentration in absence of S9 was $65 \mu \mathrm{g} / \mathrm{mL}$. Although criteria for the determinations a positive response in the lymphoma assay remained controversial according to the criteria used by the laboratory that conducted in these studies (Clive et al., 1979; Moore et al., 2003). These results can be interpreted as week toxicity. With longer exposures (about 24 hours), higher capsaicin concentrations were too toxic for analysis and the mutant fractions were not significantly different from the controls at the usable concentrations. Thus, it can be concluded that capsaicin is non-mutagenic at concentrations up $28 \mu \mathrm{g} / \mathrm{mL}$ after $24 \mathrm{~h}$ exposure in the absence of S9.

In the in vivo micronucleus study in mice, the frequency of MN-PCEs was $0.04-0.10 \%$ in the treated group compared to $0.07-0.11 \%$ in the vehicle treated control mice. Both were within the historical control range, which was started to be $0.07 \pm 0.08 \%$. The frequency of MN-PCE 
for the positive control (50 mg/kg of cyclophosphamide) was $1.5 \%$. However, in this study a difference was observed between the maximum tolerated doses estimated for males $(800 \mathrm{mg} /$ $\mathrm{kg}$ ) and females $(200 \mathrm{mg} / \mathrm{kg})$. These values are higher than previous reports in the literature (Glinsukon et al., 198O; Saito and Yamamoto, 1996), which probably may reflect the quality of the current drug substance, and do not reflect to sex difference.

Capsaicin was evaluated for its ability to induce clastogenicity in cultured human lymphocytes with and without an exogenous metabolic activation system. Clastogenecity was evaluated at concentrations that induced severe toxicity to no toxicity. Cultures were harvested within 22 hours from the initiation of treatment. Capsaicin did not induce structural and numerical chromosomal aberrations.

It can be concuded from the rsults of genotocity observations with pure trans-capsaicin that its genotocity potential is very limited and differs when the impured capsaicin or chilli extracts were used in assays.

These data have important implications for analysis of risks associated with dietary or environmental capsaicin exposures. Although the majority of epidemiological data suggests that dietary capsaicin consumption is not associated with enhanced risk of cancer (Surh and Lee, 1995). It is true that a positive matematical correlation can be observed between the intake of Chilli pepper and the gastric cancer in Mexico (Lopez-Carrilo et al., 2003), other factors than capsaicin should be investigated as the causal link in such epidemiological evaluation (Table 9).

\begin{tabular}{|c|c|c|c|c|}
\hline Test compound & Animal/Cells tested & $\begin{array}{l}\text { Hepatic S9 for metabolic } \\
\text { activation }\end{array}$ & Endpoint & Response \\
\hline CAP, Chilli & S. typhimurium & Aroclor 1254-induced rat & $\mathrm{His}^{+}$reversion & + \\
\hline CAP, Chilli & Chinese hamster V79 & Aroclor 1254-induced rat & Azaguanine resistance & - \\
\hline CAP, Chilli & Swiss mice & In vivo & Micronuclei formation & + CAP(only) \\
\hline CAP & S. typhimurium TA98 & Aroclor 1254-induced rat & $\mathrm{His}^{+}$reversion & + \\
\hline CAP, DHC, & typhimurium TA98, & Aroclor 1254-induced rat & $\mathrm{His}^{+}$reversion & - \\
\hline Chillis & TA1535 & & & \\
\hline CAP, DHC, Chilli & Chinese hamster V79 & & Azaguanine resistence & + \\
\hline CAP, Chilli & S. typhimurium & Phenobarbital-induced rat & $\mathrm{His}^{+}$reversion & - \\
\hline CAP, Chilli & S. typhimurium & & Streptomycin-resistance & $+($ Chilli); -(CAP) \\
\hline $\mathrm{CAP}, \mathrm{DHC}$ & S. typhimurium TA98, & + (source unclear) & $\mathrm{His}^{+}$reversion & - or + (CAP) \\
\hline Chilli & TA100 & & & \\
\hline Chilli & Mouse bone marrow & In vivo & Micronuclei formation & + \\
\hline CAP & Albino mice & In vivo & Pregnancy frequency & - \\
\hline CAP & Mouse epididymis & In vivo & Sperm abnormality & - \\
\hline CAP & Human lymphocytes & & Chromosome aberrations & + \\
\hline
\end{tabular}

CAP, capsaicin; DHC, dihydrocapsaicin; Chilli, planet extract (after Surh J., Lee S.S. Life Science 56: 1845-1855, 1995) (with modification)

Table 9. Genotoxicity of chilli extracts and its major pungent constituents capsaicin and dihydrocapsaicin up to 1995 


\subsubsection{Chronic toxicity studies in animals}

The chronic toxicological studies are absolutely required to drug candidate in species (one from the rodents and $\mathrm{dog}$ ) for 6 months time period. None of these types of observations could be found in the literature, consequently these studies should be done in the fourthcoming time with our preparation.

\subsubsection{Brief summary of the main results of observations with capsaicin in animals}

Capsaicin is a very active compound acting at the levels of capsaicin sensitive afferent nerves. Capsaicin has dual action, namely given in small doses it produces a biological significant gastrointestinal mucosal protective effect, meanwhile it enhances the gastrointestinal mucosal damages to different chemical, osmotic and pressure stimuli.

It is important to note that the doses (10 to $100 \mu \mathrm{g} / \mathrm{kg}$ ) produce gastrointestinal mucosal defensive actions are significantly lower than those $(100-200 \mathrm{mg} / \mathrm{kg})$ induce gastrointestinal mucosal damage.

The capsaicin is absorbed well from gastrointestinal tract in animals, and its metabolization is carried out by the liver (in pathways of enzymatic oxidation and non enzymatic oxidation). The production of epoxyns (arene) is suggested; however, it was not clearly proven.

There is specific and important observation that the capsaicin dose-dependently prevents the $\mathrm{CCl}_{4}$-induced hepatic injury during one week treatment.

The genotoxicity studies indicated a very limited positivity, which dominantly depends on the extents of capsaicin purity from plants.

No chronic toxicological studies have been published in two species (rodent, dog) and applied for 6 months.

\subsection{Human observations with capsaicin}

\subsubsection{Observations with capsaicin in healthy human subjects}

The capsaicin studies were carried out from 1997, by the permission of Regional Ethical Committee of Pécs University, Hungary. These studies were carried out in randomized, prospective manner, respecting the Helsinki Declaration. The observations were carried out according to the Good Clinical Practice (GCP), at the same methods as those are required to classical drug (or drug candidate) studies.

The First Department of Medicine, Medical and Health Centre, University of Pécs, Hungary, is one of the Hungarian Accreditated Centres for the studies of the human phase I-II. examinations. This institute has been participating in the drug developments since 1968 . 


\subsubsection{Dose-response curves of capsaicin in the human stomach acute observations}

The dose-response curves were identified on the gastric basal acid secretion (BAO) in healthy human subjects, and on the measurements in gastric transmucosal potential difference (GTPD) without and with topically (intragastrically) applied ethanol (Mózsik et al., 2005). We tested 100, 200, 400 and $800 \mu \mathrm{g}$ capsaicin dissolved in 100 saline solution given intragastrically via nasogastric tube on the gastric BAO values and on GTPD.

In other series of observations, the gastric microbleeding was produced by orally given indomethacin ( $3 \times 25$, plus $25 \mathrm{mg}$ at the starting of examinations) in healthy human subjects. Indomethacin was given alone, or in combination of capsaicin (200, 400 and $800 \mu \mathrm{g})$. The results were compared with the results obtained without application of indomethacin (baseline).

The effect of capsaicin given in smaller dose than $100 \mu \mathrm{g}$, no effect was observed. The $\mathrm{ED}_{50}$ value was obtained in experiments using $400 \mu \mathrm{g}$ dose on the gastric BAO, GTPD (without and with topically applied ethanol), and Indomethacin-induced gastric microbledings (Mózsik et al., 2005; Mózsik et al., 2007).

It was also observed that gastric microbleeding produced by both inhibition of COX-1 and COX-2 was completely prevented by the application of $400 \mu \mathrm{g}$ capsaicin (Mózsik et al., 2007; Sarlos et al., 2003).

When capsaicin was given in dose of $\mathrm{ED}_{50}$ intragastrically, the "parietal component" decreased $(P<0.001)$, meanwhile the "non-parietal component" (buffering secretion) and gastric emptying increased significantly $(P<0.001)$ in human healthy subjects (Mózsik et al., 2004; 2005; 2007; Debreceni et al., 2001). Recently, it was observed that capsaicin (given in dose of $400 \mu \mathrm{g}$ orally) enhanced the glucose absorption and glucagon release during standard glucose loading test in human healthy subjects (Dömötör et al., 2006).

\subsubsection{Changes in laboratory parameters and complaints of human healthy subjects during the study with capsaicin}

No systemic laboratory changes were noted in the biochemical parameters except the observations with gastric juice (Mózsik et al., 2004; 2005), glucose loading test (Dömötör et al., 2006). No subjective complains were observed in patients (pain, diarrhoea, vomit).

\subsubsection{Subchronic observations with capsaicin in human healthy subjects}

\subsubsection{Two weeks treatment with capsaicin}

The group of healthy human subjects received capsaicin treatment for two weeks $(3 \times 400 \mu \mathrm{g}$ given orally) in prospective, randomized study. The gastric microbleeding was produced by indomethacin application before and after the two-week capsaicin treatment. At baseline indomethacin-induced gastric microbleedings applied without and with different doses of capsaicin were measured before and after two-week capsaicin treatment. 
No changes were obtained at baseline, indomethacin-induced gastric microbleding, and on the other hand, the gastric mucosal protective effects of capsaicin remained the same after the two-week capsaicin treatment as those were found at baseline (Mózsik et al., 2005; Mózsik et al., 2007a).

\subsubsection{Biochemical meaurements and complaints in human healthy subjects during two weeks capsaicin treatment}

No changes were noted in the biochemical parameters and no complaints registered in the human healthy subjects.

\subsubsection{Human chronic observations with capsaicinoids}

In a case-control study in Mexico City included 220 cases of gastric cancer and 752 controls randomly selected from the general population. Chilli pepper consumers were found to be having a 5.5 fold greater risk for gastric cancer than non-consumers. Persons who stated themselves as heavy consumers of chilli peppers were at a 17 fold greater risk. However, when chilli consumption was measured as frequency per day, a significant dose-response relationship was not observed (Lopez-Carrillo et al., 1994).

In another case-control study in India, red chilli powder was found to be a risk factor for cancer of the oral cavity, pharynx, esophageus and laryngx compared with population controls, but not with hospital controls (Notani and Jayant, 1987).

In an Italian case-control study, chilli was briefly mentioned as being protective against stomach cancer (Buiiatti et al., 1989). Chilli pepper, however, are not heavily consumed in Northern Italy, where this study was conducted, and it is possible that chilli consumption rather correlated with other used protecting spices such as onions and garlic, which are heavily consumed in Italy.

The Committee of Experts on Flavouring Substances of the Council of Europe concluded that the available data do not allow to establish a safe exposure level of capsaicinoids for foods (Opinion of the Scientific Committee on Food on Capsaicin, adopted on 26 February, 2002).

It was also observed that the non-selective COX-1 and COX-2 inhibiting nonsteroidal antiinflammatory drug-induced gastric microbleeding can completely be prevented by $400 \mu \mathrm{g}$ capsaicin administrations in human healthy subjects (Mózsik et al., 2007).

4.4.4. Preventive effects of capsaicin against the selective and nonselective inhibitory actions produced by nonsteroidal anti-inflammatory drugs on COX-1 and COX-2 enzymes

The indomethacin (as non selective COX inhibitor) was used to provoke gastric microbleeding in healthy human subjects, and capsaicin itself, as a specific stimulator of capsaicin receptor (TRVP1) was applied in small doses to healthy human subjects. The capsaicin treatment was carried out for two weeks, it was given in 3 x 400 $\mu$ g dose orally daily (400 $\mu$ g capsaicin dose 
was obtained to be equal to $\mathrm{ED}_{50}$ value in previous human observations) (Mózsik et al., 1999; 2004; 2005) (Table 10-11).

\begin{tabular}{cc}
\hline NSAID & Ratio COX-1: COX-2 \\
\hline Aspirin & 0.12 \\
\hline Diclofenac & 38.00 \\
\hline Etodolac & 179.00 \\
\hline Ibuprofen & 0.86 \\
\hline Indomethacin & 0.30 \\
\hline Loxoprofen-SRS & 3.20 \\
\hline NS-398 & 1263.00 \\
\hline Oxaprozin & 0.061 \\
\hline Zaltoprofen & 3.80 \\
\hline
\end{tabular}

*After Kawai, S. et al. Eur. J. Pharmacol 347: 87-94 (1998)

Table 10. Comparison of inhibitory effects $\left(\mathrm{IC}_{50}\right)$ by giving the COX-1 and the various NSAIDs using human platelet COX-1 and synovial cell COX-2*

- IC I0 $_{5}$ VALUE OF INDOMETHACIN TO RATIO OF COX-1/COX-2 = 0,30

(1: 3.25)

- MICROBLEEDING IN THE STOMACH

\begin{tabular}{|c|c|c|}
\hline & $\underline{2 \text { weeks capsaicin treatment }}$ & $\longrightarrow$ \\
\hline & Before & After \\
\hline $\begin{array}{l}\text { Baseline } \\
\text { After IND }\end{array}$ & $\begin{array}{l}2,1 \pm 0,1 \mathrm{~mL} / \text { day } \\
8,25 \pm 0,25 \mathrm{~mL} / \text { day }\end{array}$ & $\begin{array}{l}2,0 \pm 0,1 \mathrm{~mL} / \mathrm{day} \\
7,8 \pm 0,3 \mathrm{~mL} / \mathrm{day}\end{array}$ \\
\hline$\Delta$ IND-induced & $6,15 \pm 0,2 \mathrm{~mL} / \mathrm{day}$ & $5,8 \pm 0,3 \mathrm{~mL} / \mathrm{day}$ \\
\hline
\end{tabular}

COX-1:1.447 $\pm 0.1 \mathrm{~mL} / \mathrm{day}$

$1.364 \pm 0.1 \mathrm{~mL} / \mathrm{day}$

COX-2: $4.70 \pm 0.2 \mathrm{~mL} / \mathrm{day}$

$4.44 \pm 0.2 \mathrm{~mL} / \mathrm{day}$

- $400 \mu \mathrm{g}$ CAPSAICIN (IG GIVEN) INDUCED DECREASE OF IND-GASTRIC MICROBLEEDING

\author{
$6 \pm 0.2 \mathrm{~mL} / \mathrm{day}$ \\ $5.9 \pm 0.2 \mathrm{~mL} / \mathrm{day}$
}

* means \pm SEM in 14 human healthy subjects.

Table 11. Correlation between the capsaicin actions, COX-1 and COX-2 systems and gastric microbleedings produced by indomethacin in human healthy subjects before and after 2 weeks capsaicin ( $3 \times 400 \mu \mathrm{g}$ orally) treatment.* 


\subsection{Summary of the observation with capsaicin alone or in combination with selectively and non-selectively inhibition of COX-1 and COX-2 enzymes by nonsteroidal anti- inflammatory compounds in animal experiments and in human observations}

The capsaicin chemically representing a mixture of compounds of capsaicinoid (capsaicin, dihydrocapsaicin, norcapsacin, nordihydrocapsaicin) has been widely used in the population nutrition of different countries for the last 9000-9500 years.

It was a significant internationally accepted discovery that capsaicin (capsaicinoids) significantly stimulates (stimulate) a subgroup of the afferent nerves (named under as "capsaicinsensitive afferent nerves") responding upon various chemical agents, heat, $\mathrm{pH}$ gradients in animal experiments and human observations.

The doses of capsaicin (capsaicinoids) are significant in the biological actions, because when it (those) is (are) given in small doses then it (those) prevents (prevent) tissue protection (including the gastrointestinal tract), however, capsaicin (capsaicinoids) enhances (enhance) the organ's damage (including the gastrointestinal tract). The capsaicin (capsaicinoids) produces (produce) organ damaging effects as a consequence of capsacin-induced desensitation proceeded by the application of capsaicin (capsaicinoids) in higher doses. The existence of these principle observations was scientifically proven in animal experiments and human observation.

The application of capsaicin has been carried out as a tool to approach the different physiological and pharmacological regulation of different diseases and their prevention.

The study of the afferent nerves has been carried out from the years of 1970. The results of this research clearly proved the principle and important role of the afferent nerves in the physiological regulation of various organs and as well as in the development of damage and prevention of these different organs.

The human observations with capsaicin were carried out randomized, prospective studies by the permission of Regional Ethical Committee of our Univesity of Pecs, Hungary. These studies were carried out in accordance of the Good Clinical Practice (GCP) together with respect of the Helsinki Declaration.

The results of the animal experiments and human observations clearly proved the gastrointestinal protection by application of small doses of capsaicin (capsaicinoids) (including the application of nonsteroidal anti-inflammatory drugs).

The anti-inflammatory drugs (acting by the properties of drugs of selective and nonselective inhibitions of COX-1 and COX-2 enzymes) are widely used in the prevention of thromboembolic episodes, in the prevention of reinfarction in patients who underwent myocardial infaction, in the treatment of patients with acute and chronic pains with degerative chronic joint diseases, malignant diseases and healthy persons for preventing gastrointestinal cancers, etc.

Capsaicinoids absorbs well from the gastrointestinal tract. They are metabolized in the pathways of enzymatic oxidation of liver and as well as non enzymatic oxidation. It is very 
important that capsaicin alone dose-dependently prevents the hepatic damage produced by carbon tetrachloride (in a significantly higher level that it was found for silymarin). The studies with genotoxicity clearly indicated that the pure capsaicin has a very limited toxicity (this value is higher if the capsaicin preparation is not chemically clear and contaminated with different toxicological agents of plants, e.g. aflatoxin, pesticides).

The human observations also clearly indicated that capsaicin actions can be reproduced well in human healthy subjects. The capsaicin application produces an dose-dependent increase in GTPD (without and with combined ethanol), decreases BAO, and totally prevents the indomethacin-induced gastric mucosal microbleedings (in range of 100 to $800 \mu \mathrm{g}$ given intragastrically). Furthermore two weeks treatment with capsaicin ( $3 x 400 \mu \mathrm{g}$ given intragastrically) did not modify the sensitivity of the gastric mucosa to capsaicin-sensitive afferent nerves and the gastric mucosal protective effects against indomethacin remained at the same dosedependent level after 2-week capsaicin treatment.

The conclusions of these animal and human observations clearly proved that the application of capsaicin in small doses is completely able to prevent the nonsteroidal anti-inflammatory drug-induced gastrointestinal side effects. By the other ways capsaicin is able to inhibit the functions of COX-1 and COX-2 enzymes in animal experiments and in human observations.

The further scientific research may offer an absolutely new pathway(s) for the development of drug (s) by the stimulation of capsaicin-sensitive afferent nerves by the usage of small doses of capsaicinoids to patients, who have to be treated with nonsteroidal anti-inflammatory drugs and for other diseases requiring cyclooxygenase inhibition (inflammations, tumors).

\subsection{Summary of expters' opinion}

The plant origin capsaicinoids (capsaicin, dihydrocapsaicin, norcapsaicin, dihydrocapsaicin, homocapsaicin, homodihydrocapsaicin) are well known and used as nutritional additive agents in the every day nutrional practice from the last 7000 years, however, we have a very little scientifically based knowledge of their chemistry, physiology, pharmacology in animal observations, and in humans up till the end of $20^{\text {th }}$ century. Our knowledge from their chemistry, physiology and pharmacology entered to be scientifically based evidence from the years of 1980s, dominantly upon animal observations. The human observations with capsaicin (capsaicinoids), in terms of good clinical practice, have been started only in the last ten years in randomized, prospective, multiclinical studies. The name of "capsaicin" used only in the physiological and pharmacological research both in animal experiments and in human observations. The "capsaicin" (as a"chemically" used natural compound) modifies the "so-called" capsaicin-sensitive afferent nerves, depending on their doses of application.

Aims: The specific action of capsaicin (capsaicinoids) on sensory afferent nerves modifying gastrointestinal (GI) function offers a possibility for the production of an orally applicable drug or drug combinations, which can to be used for human medical therapy. The production of a new drug needs to be based on the critical interdisciplinary review of the results obtained with capsaicinoids. 
Materials and methods: This paper gives an interdisciplinary and critical overview on the chemical, physiological, pharmacological and toxicological actions of the natural origin capsaicinoids (from the point of drug production) under conditions of acute, subacute and chronic administration in animal experiments and human observations for toxicology and pharmacokinetics.

This interdisciplinary review covers the following main chapters: 1. Physiological and pharmacological research tool by capsaicin in the animals and human beings; 2 . capsaicin research in animals (including the acute, subacute and chronic toxicology, metabolism as well as genotoxicology); 3. Pharmacological observation with capsaicin in human beings.

Conclusion: 1. The capsaicin used in the physiological and pharmacological observations (in animals and human beings) chemically represents heterogenous chemical compounds, which can be obtained from the plants (paprika, chilli, etc). 2. Capsaicinoids are able to modify the capsaicin-sensitive afferent nerves, which play principle roles in the defence of various organs (including the gastrointestinal tract (against heat, stress, chemical-induced damage). 3. The beneficial effects of capsaicin (capsaicinoids) application on gastrointestinal tract obtained in animal experiments can be converted for humans observations. After this interdisciplinary and critical review, this paper demonstrates well-planned research pathways of discoveries on capsaicinoid chemistry, physiology, pharmacology and toxicology in animal experiments and human observations.

\section{Some general scientific problems in the application of the plant origin compounds in the every used foods and drugs}

The evaluation of effectiveness and safety of chemically produced compound(s) is very strickly regulated testing program both in animals and in humans.

After very careful and critical overview of plant origin compounds, it was very surprising to see that health and scientific requirements differ so much in regards of their application as dietary (Response to EMEA Consultation Document CPMP/QWP/2819/00 REV 1 AKA EMEA/ CVMP/814/00 REV 1: Guideline on Quality of Herbal Medicinal Products/Traditional Herbal Medicinal Products (Released 21 July 2001/Consultation Date 30 ${ }^{\text {th }}$ September 2005) and as a drug terapy (this Notice to Applicants (NTA) prepared by the European Commission in consultation with the competent authorities of the Member States, the European Medicines Agency and interested parties in order to fulfil the Commission's obligations with respect to article 6 of Regulation (EC) No. 726/2004, and with respect to the Annex I to Directive 2001/83/ EC as amended (Directive 2003/63/EC, OJ L 159 27.6.2003 p.46 NTA, Vol. 2B-CTD, foreword \& introduction, edition June 2006).

We, the authors of this review could not understand the extremely high number of application of plant origin compounds needed to be applied for foods, food additive agents, health modification compounds and classical drugs (especially orally applicable preparations). 
A lot of chemical compounds are used during the culturation of different plants, which are be used as sources of various compounds of food or drug preparations. Furthermore, during the preparation of the cultivated plant are treated with different chemicals to result aimed chemical compounds (we can use only "Drugs Master File", surprisingly up to now no "Food Master File"). These aspects are remained out off the scientific area up till present time.

The medical sciences emphasize the prevention of different diseases. Our main question is why these aspects remained out of the scope of science?

In case of capsaicin, we have the following main problems:

1. The research of physiology and pharmacology only "capsaicin" is mentioned all the time, while the capsaicin as a plant source compound chemically does not represent one chemical entity;

2. The content of capsaicin (Sigma-Aldrich, USA) is also not standard, because its content of capsaicin, dihydrocapsaicin, nordihydrocapsaicin and other capsaicinoids can vary;

3. No correct Drug Master File (DMF) for capsaicin (capsaicinoids) has been prepared;

4. No classical animal toxicological (including the germinative function) examinations have been carried out for capsaicin (capsaicinoids);

5. No classical preclinical dossier exists for capsaicin;

6. No classical human clinical pharmacological study (human phase I-II) exists in the international literature.

Our research team works in capsaicin research from 1980 using mostly animal experiments, but starting form 1997 we are studiing capsaicin physiology in human investigations (under permission of the the Regional Ethical Committee of Pécs University, Hungary).

We actively participated in innovative research of capsaicin (capsaicinoids) to produce a new drug or new drug combinations affecting capsaicin sensitive afferent nerve function offering an absolutely new gate for gastrointestinal pharmacology (Mózsik et al., 2009a,b; 2010)

\section{Chemical composition of capsaicinoids originated from plants and their botanical backgrounds}

In physiological and pharmacological research capsaicin is generally used as "one chemical compound". Capsaicin (capsaicinoids) is (are) active chemical substance(s) extracted from paprika, chilli, chillies, which is (are) able to modify the capsaicin-sensitive afferent nerves. This (these) compound(s) stimulates (stimulate) in smaller doses and inhibits (inhibit) in higher doses of capsaicin-sensitive afferent nerves.

Szolcsányi and Barthó demonstrated first that capsaicin given in doses of 5-50 $\mu \mathrm{g} / \mathrm{mL}$ inhibits the development of gastrointestinal mucosal damage (Szolcsányi and Barthó, 1981). Similar 
results were obtained in systematic research on gastrointestinal tract in animals (Mózsik et al., 1997) and human healthy subjects treated with indomethacin (Mózsik et al. 2005; Mózsik et al., 2009a,b).

The action of capsaicin expresses itself as an initial short-lasting stimulation that can be followed by desensitation to capsaicin itself and to other stimuli of afferent sensory neurons. Capsaicin applied in ng to $\mu \mathrm{g} / \mathrm{kg}$ doses to the peripheral or central endings or cell bodies of sensory neurons induces transient excitation. In response to stimulation peptide mediators are released from the central and peripheral nerve endings (Szolcsányi, 1984; Maggi, 1995). These four response stages could be separated: 1. excitation, 2. sensory-blocking, 3. long-term selective neurotoxic impairment and 4. irreversible cell destruction. From the point of gastrointestinal mucosal protection only the small doses of capsaicin have clinical relevancy (these doses 200-1200 Mg/person) (Mózsik et al., 2005; Mózsik et al., 1997; Mózsik et al., 2009a,b; 2010, Szabó et al., 2013).

Seven capsaicinoids and their chemical structures have been identified from different Capsicum species: C. annuum; C. frutescens; C. chinese; C. baccatum; C. pubescens) (Table 9) (Basu and De, 2003; Anu and Peter, 2000; Jurenitsch et al., 1979): capsaicin, dihydrocapsicin, nordihydrocapsaicin, homodihydrocapsicin; homocapsaicin; nonanoic acid vanillylamide and decanoid acid vanillylamide (Basu and De, 2003, Anu and Peter, 2000; Jurenitsch et al., 1979). The first five compounds represent capsaicin homologues, meanwhile the last two represent capsaicin analogues (Fig. 16).

The fine chemical trading firms (including Sigma Aldrich and others) obtain the capsaicin from plants (Capsicum). According to the chemotaxonomic key, the different species of Capsicums contain different amounts of capsaicin homologues and analogues (Jurenitsch et al., 1979) (Table 13).

\begin{tabular}{lllll}
\hline Species & Flower colour & Number flw/node & Seed colour & Calyx constriction \\
\hline C. annuum & white & 1 & $\tan$ & absent \\
\hline C. frutescens & greenish & $2-5$ & $\tan$ & absent \\
\hline C. chinese & white/greenish & $2-5$ & $\tan$ & present \\
\hline C. baccatum & white with yellow spot & $1-2$ & $\tan$ & absent \\
\hline C. pubescens & purple & $1-2$ & black & absent \\
\hline
\end{tabular}

* For further informations, see Ref. Mózsik et al.(2009b)

Table 12. The morphological identification of the five major species* 
<smiles>[R]C(=O)NCc1ccc(O)c(OC)c1</smiles>

Name

Capsaicin

Dihydrocapsaicin

Nordihydrocapsaicin

Homocapsaicin

Homodihydrocapsaicin

Nonanoic acid vanillylamide

Decanoic acid vanillylamide
Structure of the "R" chain
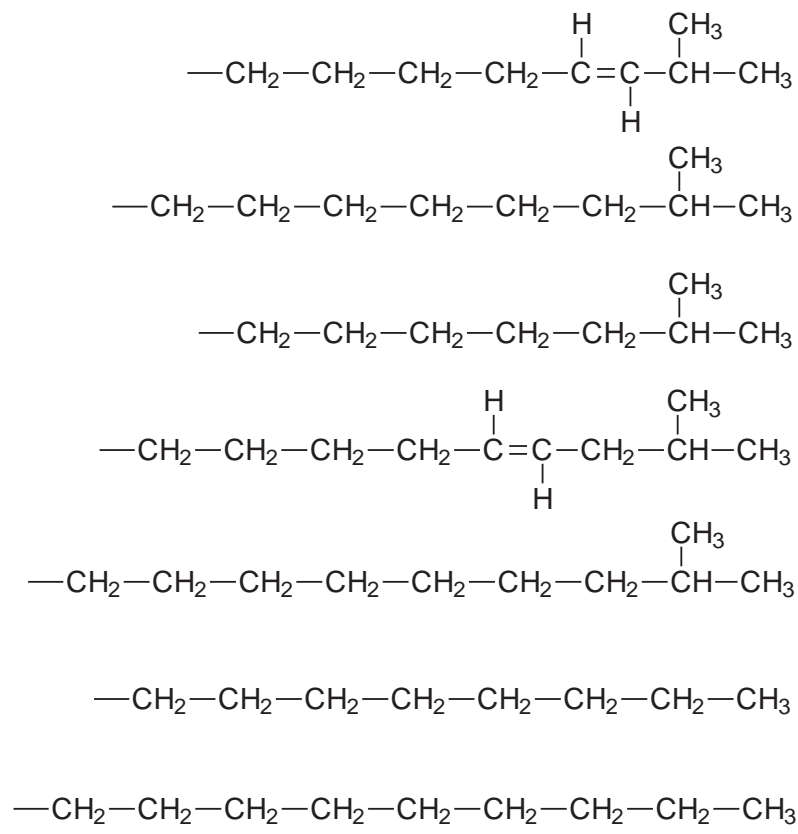

Figure 16. Structure of capsaicin and natural capsaicinoids (Mózsik et al, 2009b).

Accordingly, the commercially available natural capsaicin preparations are mixtures of capsaicin and natural capsaicinoids (Fig. 17).

The capsaicin preparation can be used as an active pharmaceutical ingredient (Capsaicin Natural) is described by the United States Pharmacopeia (USP). The 2006 edition of USP30NF25 described its definition, identification, melting range and the content of capsaicin, dihydrocapsicin and other capsaicinoids. According to the USP requirements Capsaicin Natural should contain not less than 90 percent of total capsaicinoids. The content of capsaicin and dihydrocapsaicin should not be less than 75 percent, and the content of other capsaicinoids should not be more than 15 percent calculated on dried basis [USP30-NF25 Page 1609].

The principal requirements of the European Authorities for capsaicinoid content of natural capsaicin preparations usable in medical therapy are the same as those of USP30-NF25. 
1. NDHC fraction over $9.5 \%$

\begin{tabular}{lll}
\hline 1.1 & Cfraction over $56 \%$ & Capsicum baccatum var. pendulum \\
\hline 1.2 & C fraction under $56 \%$ & Capsicum annum var. annum \\
\hline 2. & NDHC C Fraction $9,5 \%$ & Capsicum annum var. annum \\
\hline 2.2 & C fraction, 42 to $57 \%$ & Capsicum baccatum var. pendulum \\
\hline 2.3 & C fraction under $42 \%$ & \\
\hline & DHC fraction, 57 to $73 \%$ & Capsicum baccatum var. pendulum 26 to $34 \%$ \\
\hline & Total capsaicinoids, under 0,35\% & \\
\hline & C fraction over $63 \%$ & Capsicum fritescens - Capsicum chinense complex \\
\hline
\end{tabular}

Abbreviations: C, capsaicin; NDHC, nordihydrocapsaicin; DHC, dihydrocapsaicin

* For further I nfromations, see Ref. Mózsik et al.(2009b).

Table 13. A chemotaxonomic key to the identification of cultivated Capsicums*

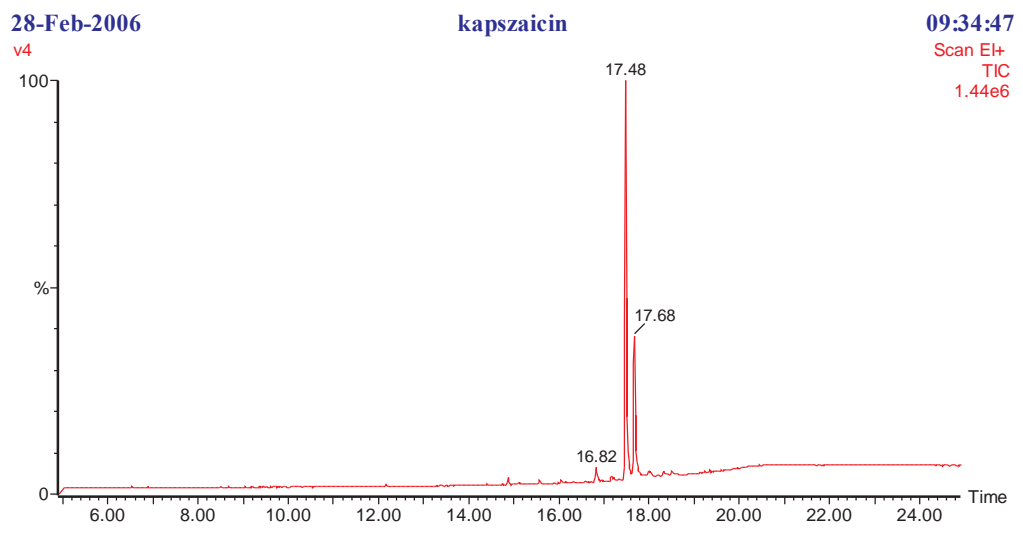

Figure 17. Gas-chromatographic (GC-MS) analysis of a commercially available natural capsaicin preparation. Retention times: $16.8 \mathrm{~min}$ : nordihydrocapsaicin, $17.5 \mathrm{~min}$ : capsaicin, $17.7 \mathrm{~min}$ : dihydrocapsaicin [36].

For pharmaceutical industry and human clinical pharmacology the presence of several components of natural capsaicin preparations represent special technological and clinical pharmacological difficulties. Namely, we have to quantify these components of capsicum extract in the pharmaceutical preparations as well as in the human biological blood samples, consequently we have to use specific and sensitive analytical methods. 


\section{Drug Master File (DMF)}

To receive permission for human use of capsaicin preparations from the National and International Regulatory Authorities we have to present the following details: 1 . specification of the Capsicum species; 2. climatic regulations of places of Capsicum cultivation; 3. chemical treatments of Capsicum plants during their cultivation; 4. details of treatment of Capsicum plants (their collections, drying, extractions storages, etc.), analytical results supporting the chemical composition of the plant origin capsaicinoids extract; 6 . chemical stability of the natural capsaicin (capsaicinoids); 7. analytical results showing the (possible) contamination of the natural capsaicin product with organic phosphates, pesticides, fusariums, aflatoxin; 8 . international certification (including Food and Drug Administration, FDA) on capsaicin (capsaicinoids) content of the natural preparation. Data of above mentioned facts need to be given by internationally accredited laboratories. These data are collected in the Drug Master File (DMF).

The leading chemical trading firms-concerning capsaicin supply-had no DMF for their capsaicin preparations. Independently, several trading firms keep the natural capsaicin (capsaicinoids) preparation on the market without the exact knowledge on the circumstances of cultivation, details of extraction and stability of the product. They have no exact information on the quantities of residues of organic phosphates, pesticides, fusariums, aflatoxin in their preparation of capsaicin (proved by certifications of various internationally accredited laboratories).

According to the observations of Foodnews Enviromental Working Group (foodnews.org;http://www.drgreene.org/body.cfm?xyzpdqabc=21\&action=detail\& ref=1920), the most sweet peppers are contaminated with more than one pesticide. Pesticides were not detected only in $32 \%$ of the samples, and 7 pesticides were observed in $1 \%$ of tested samples. The samples of Sweet Bell Peppers contain acephate, dicophol, dimethoate, diphenylamine, fenvalerate, metalaxyl, methamidophos, methomyl, fermethrin; malathion, endosulfanes, azinphosmethyl, o-phenylphenol, which may produce animal carcinogens, birth defects, brain and nervous system damage as well as the damage of immune system and endocrine system (Report Card www.ewg.org).

In our case we found only one natural capsaicin preparation with Drug Master File (DMF) from India, which signed by the Food and Drug Administration, USA. Along with this preparation, we could not exact information from the manufacturer on above mentioned data to be incorporated into the DMF.

\section{Preparation of human clinical pharmacological studies}

We wanted to use capsaicinoid preparation as an orally applicable drug or part of a drug combination in human beings. 
We compiled the below listed documentations for the National Institute of Pharmacy to apply permission for human clinical pharmacological studies with capsaicin preparation

1. experts' opinion; 2. results of all toxicological studies; 3 . chemical stability of the natural capsaicin preparation; 4 . results of pharmaceutical industrial formulation from the natural capsaicin; 5. various permissions from our University; 6. documentation of health insurance of volunteers; 7. preclinical dossiers; 8 . documented valid permission on the accreditation of Clinical Pharmacological Unit for human phase I and II examinations (accreditation controlled by the National Institute of Pharmacy in Hungary); 9. exact protocols for human clinical pharmacological studies; 10 . written information on the planned examinations for the volunteers; 11. request for authorization of a clinical trial on medical product for human use to the competent authorities and ethical committees in the community; 12. lists of investigators (together with their CV), data of involved institutes (departments participating in the study).

\section{The National Instute of Pharmacy in Hungary requested additional exmainations with natural capsaicin (capsaicum natural usp 27) obtained from India on geno-and other toxicological examinations}

The National Institute of Pharmacy in Hungary requested additional examinations with natural capsaicin (Capsaicin Natural USP 27) obtained form India on geno-and other toxicological studies due to having limited knowledge of circumstances of cultivation, collection, storage, stability and preparation. In the literature some data were available supporting genotoxic property of some natural capsaicin preparations by different researchers. Some positivities were indicated with natural capsaicin on its genotoxicity and the different researchers suggested that these mostly depend on various enviromental factors of natural capsaicin, since these tests were negative with synthetic capsaicin.

These requisted studies with the natural capsaicin obtained from India were 1. testing of natural capsaicin with bacterial reverse mutation assay; 2 . testing of mutagenic effect of natural capsaicin by mouse micronucleus test; 3 . 14-day oral average dose range finding study with natural capsaicin in rats (30-60 and $120 \mathrm{mg} / \mathrm{kg}$. b. w. orally for 14 days); 4 . oral dose range finding toxicity study of natural capsaicin in Beagle dogs $(0.3-0.6-0.9 \mathrm{mg} / \mathrm{kg}$. b. w. /day orally for 14 days); 5. 28-day oral toxicity study of natural capsaicin in rats (placebo, 5, 15 and $30 \mathrm{mg} / \mathrm{kg} \mathrm{b.} \mathrm{w} \mathrm{orally} \mathrm{for} 28$ days); 6. 28-day oral toxicity study of test item natural capsaicin in Beagle dogs (placebo, $0.1-0.3-0.9 \mathrm{mg} / \mathrm{kg} \mathrm{b}$. w/day orally for 28 days) (together with capsaicin kinetics).

Determination of LOD and LOQ (Reilly et al.,2002).: Limit of detection (LOD) was determined experimentally, and taken as the concentration producing a detector signal that could be clearly distinguished from the baseline noise (3 times baseline noise). The limit of quantification (LOQ) taken as the concentration that produced a detector signal ten times greater than the baseline noise. The LOD and LOQ values of capsaicin and dihydrocapsaicin in dog's plasma were found to be $2 \mathrm{ng} / \mathrm{mL}$ and $10 \mathrm{ng} / \mathrm{mL}$, respectively. 
The sensitivity of the present method exceeds that of the HPLC-MS methods previously reported for determination of the two main capsaicinoids in rat plasma and tissues (Reilly et al., 2002). Furthermore, the method is practical and less expensive than current methodology.

In our experience, after per os administration of Capsaicin natural (USP 29) in dogs neither capsaicin nor dihydrocapsaicin could be detected in the plasma samples. Our HPLC-FLD results were confirmed by investigation of the samples by HPLC-MS. Ex vivo animal investigation of pharmacokinetics of per os administered capsaicinoids are under way by means of the present method for better understanding of the gastrointestinal fate of capsaicinoids.

In the 2005-2008 time period our innovative drug research produced the following main subjects: 1 . developed and validated methods for testing of drug active agents, and for testing of biological samples: 7 methods; 2 . developed of a validated liquid chromatography-mass spectrometry (LC-MS) method for testing of drug(s) (and their metabolites) in biological samples; 3. developed other validated analytical protocols: 7 protocols; 4 . validated genotoxicity examinations were carried out with natural capsaicin; 5 . internationally validated 14-day oral gavage range finding studies were carried out with natural capsaicin in rats and dogs; 6 internationally validated complete 28-day oral toxicity studies were carried out with natural capsaicin in rats and Beagle dogs (Mózsik et al., 2008 a,b,c,d,e,f,g,h).

After compilation of the results of these observations we received permissions from the National Institute of Pharmacy in Hungary for human phase I. clinical pharmacological studies for natural capsaicin (Capsaicin Natural USP27) used alone and natural capsaicin plus NSAID combinations. Our industrial partner was PannonPharma Ltd., Pécsvárad Hungary, who did the pharmaceutical industrial research in the field.

\section{Human phase I. single-blind study comparing the pharmacokinetic properties of asa and its platelet aggregation after single administration alone and co-administration with two different doses of capsaicin (400 and $800 \mu \mathrm{G})$ and evaluating their safety in healty male jubjects}

(protocol number: 1.4.1; EudraCT number: 2008-007048-32)

\subsection{Main aims of these studies}

We planned to produce various drugs combinations, in which ASA, diclofenac, Naproxen (as NSAIDs) were combined with capsaicin(oids) in tablet [suggesting that the NSAIDs induced GI mucosa damage can be prevented by the capsaicin(oids)].

1. To plan the chemical compositions of these drug combinations for their pharmaceutical industrial productions (dosages, bioadhesive compounds of tablets) and to produce them. We wanted to start with a human clinical pharmacological phase I. examinations (respective all the national and international experts' requirements and necessary permissions form different (18) Authorities) (Mózsik et al., 2010). 
2. We wanted to study whether the capsaicin(oids) (orally given in different doses, which only stimulate the capsaicin-sensitive afferent nerves) is(are) able to modify the absorption, metabolism, excretion of NSAIDs and their specific pharmacological actions (e.g. the platelet aggregation in case of ASA) in human healthy subjects.

This book chapter deals with the problems and results of ASA+capsaicin(oids) combination in healthy male subjects during "classical human pharmacological phase I. examinations".

Inclusion and exclusion of so-called healthy persons for the human phase I. examination (according the principals of clinical pharmacology) and characterizations of different somatic parameters of included healthy subjects into the phase I. study,

3. Schedule of the protocol for whole study (including the clinical phase, detections of capsaicin(oids), measurements of ASA - and salicylic acid (as one of the metabolites) of ASA and platelet aggregation produced by capsaicin(noids) (given in two doses) alone or in combination with ASA),

4. The registrate the tolerance and safety of these combinations in these clinical pharmacological studies,

5. Definitive results of measurements of plasma capsaicin and dihydrocapsaicin, and their evaluation,

6. Pharmacokinetic measurements of ASA, salicylic acid after application of ASA alone and in combinations with capsaicin(oids) (given orally in two doses),

7. Platelet aggregation studies with capsaicin(oids) alone and capsaicin(oids) in combination with ASA.

\subsection{Clinical pharmacological aspects of the planned examinations: pharmaceutical preparation of tested preparations of tested drugs}

1. ASA (acidum acetylsalicylicum 500, manufactured by PannonPharma Ltd, Pécsvárad, Hungary);

- Active ingradient: acidum acetylsalicylicum,

- Batch number: F005/2008-4,

- Formulation: Tablet.

2. Capsaicin(oids) (capsaicin $400 \mu \mathrm{g}$ manufactured by PannonPharma Ltd, Pécsvárad, Hungary);

Formulation: film-coated tablet,

Active ingradient: capsaicin (Capsaicin USP as manufactured in Andhra Pradesh, India, and Drug Master File was assigned by Drug and Food Aministration in USA: “17856 A II 26.10.2004 Asian Herbex Ltd“ for as orally applicable drug substance in humans),

3. Placebos (indentical with capsaicin film-coated tablet and with acetylsalicylic acid tablet, manufactured by PannonPharma Ltd., Pécsvárad, Hungary). 
The following doses of drugs were studied in this human phase I. examination:

- ASA : $500 \mathrm{mg}$,

- Capsaicin(oids) : $400 \mu \mathrm{g}$ and $800 \mu \mathrm{g}$,

- ASA placebo,

- Capsaicin(oids) placebo.

"Human phase I. single-blind study comparing the pharmacokinetic properties of ASA after single administration alone and co-administration with two different doses of capsaicin (400 and $800 \mu \mathrm{g}$ ) and evaluating their safety in healthy male subjects"

Protocol number: 1.4.1

EudraCT number:2008-007048-32

The study for human clinical phase I. examination was permitted by

the Hungarian Institute of Pharmacy (Budapest, Hungary) (dated by June 4, 2009),

the National Ethics Commitee for Clinical Pharmacology, Medical Research Council (Budapest, Hungary) (dated by March 11, 2009).

The clinical pharmacological study was carried out at Clinical Pharmacological Unit of the First Department of Medicine, Medical and Health Centre, University of Pécs, Hungary.

The pharmacokinetic measurements were done at PannonPharma Pharmaceutical Ltd., Pécsvárad, Hungary.

The initiation date: March 10, 2011 and finished (including the clinical examinations, pharmacokinetic measurements, mathematical analysis, closing of written reports) by December 6, 2012.

[Selected references to the preparation of protocols:

1. Declaration of Helsinki (1964) as revised in Tokyo (1975), Venice (1983), Hong Kong (1989), Somerset West, RSA (1996) and Edinburg, Scotland (2000) with the Note of Clarification on Paragraph 29,Washington (2002),

2. ICH Topics E3. Structure and Content of Clinical Study Reports. Step 4, Consensus Guidelines from 30,11, 1995.Note for Guidance on Structure and Content of Clinical Study Reports (CPMP/ICH/137/95). July 1996.

3. ICH Topics E6. Guidelione for Good Clinical Practice. Step 5. Consolidated Guideline from 01.05.1996.Note for Guidance on Good Clinical Practice (CPMP/ICH/135/95). January 1997.

4. R.H.B. Meyboom, Y.A. Hekster, A.C.G. Egberts, F.W.J. Gribnau, R. Edwards: Causal or Causal? The Role of Causality Assessment in Pharmacovigilance - Drug Safety. 17.12.1997. 
5. ICH Topic E2. A Clinical Safety data Management: Definitions and Standards for Expediting Reporting Step 5. 01.06.1995. Note for Guidance on Clinical Data Management: Definitions and Standards for Expecditing Reporting (CPMP/ICH/377/95),

6. ICH Topic E9. Statistical Principles for Clinical Trials Step 4. Consensus Guideline 05.02.1998. Note for Guidance on Statistical Principles for Clinical Trials (CPMP/ICH/ 363/96),

7. CPMP/PhVWP (III/3445/91): Causality classification in pharmacovigilance in the European Community.]

10.3. Requested permissions from the different authorities before the start of human phase I. examinations

We compiled the documentations for the National Institute of Pharmacy in Hungary to ask for human clinical pharmacological studies with this capsaicin preaparation (all the documents listed below were requested for receiving permissions):

1. Experts' opinion (Mózsik et al., 2007b; Mózsik et al.,2009b'),

2. Results of all toxicological studies,

3. Chemical stability of the natural capsaicin preparation,

4. Results of pharmaceutical industrial formulation from the natural capsaicin,

5. Different permissions from University (Regional Ethics of Committee),

6. Documentation of health insurance of volunteers,

7. Preclinical dossiers (protocols for the planned clinical pharmacological studies),

8. Documented valid permission on the accrediation of Clinical Pharmacological Unit for human phase I. and II. examinations - which accrediatation controlled by the National Institute of Pharmacy - was prepared for the National Institue of Pharmacy in Hungary,

9. Exacts protocols for the human clinical pharmacoligical studies,

10. Written information on the planned examinations, for the volunteers,

11. Request for authorization of a clinical trial on medical product use to the competent authoririties and for of opinion of the ethical committees in the community,

12. Lists of investigators (together with their CV), place institutes (departments) participating in the study and of course, the all written agreements and permissions.

10.4. Collection, screening of the healthy male volunteers Selection on volunteers:

- In this study fifteen healthy male subjects, age between $18-55, \mathrm{BMI} 18-29.9 \mathrm{~kg} / \mathrm{m}^{2}$ were involved, 
- A person is healthy if medical examination did not find any pathological signs and other screening test parameters stated in this protocol were within the normal range and the subject did not mention any significant disease when taking the case history,

- If, in the course of initial screening, some pathological values would be observed, these findings had to be regarded as an exclusion criterion. Having a laboratory parameter out of the normal range could generally not regarded as an exclusion criterion provided that:

- they were not accompanied by clinical symptoms,

- the context of related laboratory values gave no indication of pathological process and

- the Investigator regarded them as clinically irrelevant in a written form in the Case Report File (CRF) from the accepted protocoll.

\section{Inclusion criteria:}

The subject could be involved in the trial if the following criteria had been fulfilled:

- Voluntary participation after given information (Informed Consent signed and dated before the start of the screening period),

- Age between 18 and 55 years,

- Healthy male subjects,

- BMI: $18-29.9 \mathrm{~kg} / \mathrm{m}^{2}$

- Negative physical status by physical examinations,

- Laboratory parameters within the normal range,

- Normal ECG findings - standard 12 leads.

\section{Exclusion criteria:}

- Unwillingness or incapacity to sign the written Informed Consent Form,

- Any clinically significant acute or chronic abnormalities during the physical examinations at screening,

- Clinically significant history or presence of any clinically significant gastrointestinal pathology (e.g. gastric or duodenal ulcer, gastro-oesophageal reflux, chronic diarrhoea, IBD), unresolved gastrointestinal symptoms (diarrhoea, vomiting),

- Clinically significant liver or kidney disease, or other condition which known to interfere with the absorption, metabolism and excretion of the study drug,

- Clinically significant cardiac and neurological disease in the medical history,

- Any clinically significant changes of laboratory tests from blood and/or urine,

- Donation of blood within 3 months prior to the study, 
- Acute infection,

- Positive virological testing,

- Pathological findings on the standard 12 lead ECG,

- Hypertension (blood pressure higher than 140/90 mmHg (systolic/diastolic),

- Heart rate outside the range of 50-100 beats per minute,

- History of psychiatric diseases and treatment,

- Known hypersensitivity to any component of the study drugs,

- Use of any drugs known to induce or inhibit hepatic metabolism (inducers for example: barbiturates, carbamazepine, phenytoin, glucocorticoids omeprazole; examples for inhibitors: SSRIs, cimetidine, diltiazem, macrolides, imidazoles, neuroleptics, verapamil, flouroquinolones, and antihistamines) within 1 month prior to study drug administration,

- Use of any prescribed medication within 14 days or over-the-counter medication within 7 days prior to study drug administration,

- Any depo injection or medication implant within 3 months prior to administration of study medication,

- Any food allergy, intolerance, restriction or special diet i.e. vegetarian, which in the opinion of the investigator could contraindicate the subject to participate in the study,

- Participation in another clinical trial within 3 months prior to this study,

- Positive screen on drug abuse,

- Positive alcohol breath test (at time of hospitalisation),

- Known drug or chronic alcohol abuse, drug addiction,

- Malignant disease,

- Smoking more than 10 cigarettes/day (or comparable),

- Excessive caffeine drinking (more than 3 cups a day),

- Legal incapacity and/or other circumstances rendering the volunteer unable to understand the nature, scope and possible consequences of the study,

- Evidence of an uncooperative attitude,

- Vulnerable subject. 
10.6. Clinical parameters of included healthy male volunteers (Table 14)

Age of subjects from 20 to 44 years (average is $34.5 \pm 6.7$ years),

Body heights form 170 to $196 \mathrm{~cm}$ ) (average is $180.8 \pm 6.9 \mathrm{~cm}$ ),

Body weights from 69.5 to $101 \mathrm{~kg}$ ) (average is $84.8 \pm 9.6 \mathrm{~kg}$ ),

Body Mass Index (BMI) from 20.77 to $29.68 \mathrm{~kg} / \mathrm{m}^{2}$ (average is $25.97 \pm 2.87 \mathrm{~kg} / \mathrm{m}^{2}$ ).

Table 14. Clinical parameters of included healthy male subjects in our human phase I. studies. (Number of volunteers=15) (average \pm SD):

10.7. Study design (Figure 18)

\section{GENERAL SCHEDULE OF OUR HUMAN PHASE I. EXAMINATION}

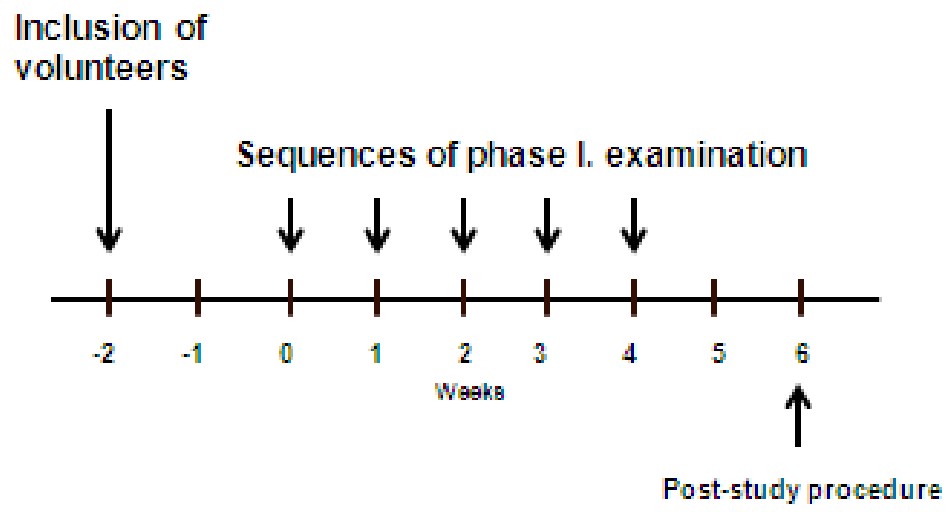

Time period of phase l. examination is maximally 8 weeks.

Figure 18. Study design of human phase I.examination of capsaicin alone or co-adminsiration with Aspirin (ASA)

10.8. Study procedures and treatment periodes - Study procedure in both treatment periods Hospitalisation of subjects:

Subjects were required to attend the Unit on the evening preceding dose administration not later than 8:00 p.m. Subjects should fast for 10 hours (9:00 p.m. - 7:00 a.m.) prior to dosing. Only water may be drunk after the evening meal until 6:00 a.m. and then no fluids will be 
taken prior dosing. Subjects had to remain in upright position (i.e. sitting, standing) for 30 minutes after dosing. Meals were provided within the Unit. Subjects were allowed to leave the clinic after the last blood sampling post dose or after blood drawing for safety laboratory tests.

\section{Daily activities during the trial:}

\section{Period 1:}

Day 0: This day was the day before administration of the study medication and had to be not later than 2 weeks after screening. Subjects had to report to the clinic at 8 p.m. on the day before dosing. The subjects received an evening snack which had to be consumed until 9 p.m.,

- Short interview for possible presence of exclusion criteria,

- Physical examination, temperature, blood pressure, pulse rate ECG,

- Alcohol breath test,

- Urine drug test was performed.

Day 1-2: The following procedures were carried out or checked prior to the drug administration in all subjects:

- Insertion of the intravenous canule, if applicable,

- Blood sampling for pharmacokinetic analysis before dose (0:00 h),

- Study drug administration between 7 and 9 a.m. according to the randomization list. Compliance check had to be performed. Subjects took the medication with $150 \mathrm{ml}$ water in upright position and remained upright for 30 minutes after dosing.

- Additional $150 \mathrm{ml}$ water had to be drunken 2 hours after drug administration,

- Blood sampling (4 ml) for pharmacokinetic analysis up to 24:00 h after administration (predose and 0:10, 0:20, 0:40, 1:00, 1:30, 2, 4, 6, 10, 12, 16, and 24 hours post dose) with separation of plasma,

- 12-lead ECG examination after the 4 hour blood sample, and before the meal,

- Standard meal 4 hours post dose,

- Questioning for and registration of adverse events.

- Wash-out period: A wash-out period of at least 3 days followed the all but one each treatment period; registration of adverse events.

- Following study periods: Procedures over 3 days (please see day 0 to 1 in period 1 ) identical with period 1 . After the last study period completed final examinations were carried out.

- Water intake: During the period from two hours prior to dose administration until after the 4 hour blood sample was taken, each subject took $150 \mathrm{ml}$ of water with the dose and $150 \mathrm{ml}$ of water after the 2 hour blood sample. No other fluid was permitted during this time. Subjects were allowed to drink water ad libitum. 
- Diet: Four hours after the drug treatment, subjects consumed a standard meal, wellbalanced in carbohydrates, lipids and proteins and resume their normal rate of fluid intake.

\section{Concomitant medication:}

- Concomitant medication was generally not allowed for the duration of the study. If this was considered to be necessary for the volunteer's welfare it could be given at the discretion of the Investigator. The volunteers had to inform the Investigator about any intake of other medicine in the course of the trial. Any intake of concomitant medication had to be documented in the Case Report Form (CRF).

- Additional intake of acetylsalicylic acid was considered as exclusion criteria.

\section{Post-study procedure:}

- Within one week after the last blood sampling time point of the last treatment period followup examinations was performed:

- Physical examination (incl.: blood pressure, heart rate, body weight, temperature), ECG,

- Laboratory test: haematology, blood chemistry and urine (after 10 hours fasting condition),

- Clinically relevant deviations of laboratory parameters (with the exception of those measured during the screening period) were regarded as adverse events.

10.9. Drug administration schedule and randomization of healthy male volunteers included into the study

\begin{tabular}{llllll}
\hline \multicolumn{5}{c}{ Treatments } \\
\hline Sequence $\mathbf{1}$ & 400 & $400+$ ASA & 800 & $800+$ ASA & ASA \\
\hline Sequence $\mathbf{2}$ & 400 & $400+$ ASA & 800 & ASA & $800+$ ASA \\
\hline Sequence 3 & 400 & $400+$ ASA & ASA & 800 & $800+$ ASA \\
\hline Sequence 4 & 400 & ASA & $400+$ ASA & 800 & $800+$ ASA \\
\hline Sequence 5 & ASA & 400 & $400+$ ASA & 800 & $800+$ ASA \\
\hline
\end{tabular}

The table above indicates only the active drugs. The remaining drugs are placebos.

It means the following:

400:1 tablet of $400 \mu \mathrm{g}$ capsaicin+1 ASA placebo+1 capsaicin placebo

$\mathbf{8 0 0}: 2$ tablets of $400 \mu \mathrm{g}$ capsaicin+1 ASA placebo

ASA: 1 tablet of ASA+2 capsaicin placebo

ASA+400:1 tablet of ASA+1 tablet of capsaicin $400 \mu \mathrm{g}+1$ capsaicin placebo

ASA+800:1 tablet of ASA+2 tablets of capsaicin $400 \mu \mathrm{g}$

Table 15. Drugs administration schedule for healthy male voluneteers 
10.10. Randomization of volunteers for this pharmacokinetic study (Table 16).

\begin{tabular}{llllll}
\hline & Sequence 1 & Sequence 2 & Sequence 3 & Sequence 4 & Sequence 5 \\
\hline \multirow{3}{*}{$\begin{array}{l}\text { Subject } \\
\text { number }\end{array}$} & 10 & 4 & 3 & 2 & 1 \\
\cline { 2 - 6 } & 13 & 8 & 11 & 9 & 5 \\
\hline
\end{tabular}

The numbers in the table indicates the order of volunteers in time of inclusion. The time of the study was five weeks ( 3 days for the kinetic measurements, 3 days for wash-out) (see later)

Table 16. Random allocation of healthy male volunteers in this study

\subsection{Chemical composition of "capsaicin" (capsaicinoids)}

The United States Pharamcopeia defines capsaicin as product with contains $>55 \%$ capsaicin, and combination of capsaicin and dihydrocapsaicin $>75 \%$; total capsaicinoids may be as little as $90 \%$ (United States Pharmacopoeia, 2005; USP 37).

This capsaicin definition is used as capsaicin in the animal researches and as well as in human observations.

The measurements of capsaicin and dihydrocapsaicin are accepted in the animal and human pharmakokinetic observations.

\subsection{Measurements of Capsaicin and Dihydrocapsaicin from the plasma of volunteers}

\section{Measurements were performed:}

- by High Pressure Chromatography (HPLC)-limit of detection by HPLC is 20 nanogram /mL plasma,

- by Liquid Chromatography - Mass Spectromery (LC-MS)-limit of detection is 26 fentogram/ $\mathrm{mL}$ for capsaicin and 20 fentogram/mL for dihydrocapsaicin.

\section{Results:}

No capsaicin and no dihydrocapsaicin could be detected in any samples of plasma of volunteers, after oral application of capsaicin (given orally in doses of $400 \mu \mathrm{g}$ and $800 \mu \mathrm{g}$ ) in time period of 0 to 24 hours. If we applied the capsaicinoids externally to the equipments, then we were able to detect both capsaicin and dihydrocapsaicin.

(Similar negativ results were obtained in Beagle dogs treated different doses $(0.1,0.3$ and 0.9 $\mathrm{mg} / \mathrm{kg} /$ body weight orally in every day for one month period) (Mózsik et al. 2008; Boros et al., 2008). 


\subsection{Pharmacokinetic measurements of ASA alone and in combination with capsaicin(oids)}

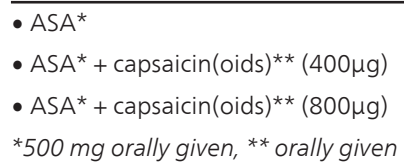

ASA metabolism in humans

by ASA esterase

ASA $\rightarrow$ salicylic acid + acetic acid

Measurements: ASA and Salicylic acid

Table 17. Combinations of pharmacokinetic measurements of human phase I. examinations

\section{Blood sampling:}

- Blood samples of $4 \mathrm{ml}$ were taken from the forearm vein at the following times: pre-dose and $0: 10,0: 20,0: 30,0: 40,1: 00,1: 30,2,4,6,10,12,16$, and 24 hours post dose,

- Blood samples (14 in each period) were taken by vein puncture. Samples (4 ml) were collected into tubes using anticoagulation agent (potassium fluorid). The total amount of blood taken from each volunteer was not exceed $500 \mathrm{ml}$,

- After taking the blood sample for pharmacokinetic analysis, it was immediately transferred to a bath of melting ice and remained there for not more than 20 minutes. After centrifugation $\left(1600 \mathrm{~g}, 4^{\circ} \mathrm{C}, 10 \mathrm{~min}\right)$, the separated plasma from each sample was divided into two aliquots (not less than $1 \mathrm{ml}$ in each tube) using transparent, polypropylene tubes and they were immediately frozen and stored at a temperature below $-20^{\circ} \mathrm{C}$ until analysis,

- Tubes were labelled. Each label contained the following information: study number, period and sample number, blood sampling time point, subject's randomisation number.

- After the end of clinical part, samples were transferred frozen directly to the analytical facility. Samples were packed with dry ice for transport, no interruption of the freeze cycle is allowed.

\section{Pharmacokinetic and statistical evaluation:}

The following pharmacokinetic parameters for the salicylic acid were calculated (by Prof. Mihály Klincsik, Department of Mathematics, Pollack Mihály Faculty of Engineering University of Pécs, Hungary) for each subject using model - independent approaches as follows:

- $\mathrm{C}_{\max }$ observed maximal concentration,

- $\mathrm{T}_{\max }$ time corresponding to the observed maximal concentration,

- $\mathrm{AUC}_{0 \text {-llast }}$ area under the plasma concentration time-curve, calculated by means of log-linear trapezoidal rule from time zero to the last data point above quantitation limit, 
- $\mathrm{AUC}_{0-\infty}$ area under the plasma concentration time-curve extrapolated from zero to infinity: $\mathrm{AUC}_{0-\infty}=\mathrm{AUC}_{0 \text {-last }}+\mathrm{C}_{\text {last, calc }} / \lambda_{\mathrm{z}}$ where $\mathrm{C}_{\text {last, calc }}$ represents the estimated plasma concentration by the regression line at the last sampling time point with measured concentration above the limit of quantitation, and

- $\lambda_{z}$ represents the rate constant calculated from the regression line,

- $t_{1 / 2}$ terminal half-life calculated from the terminal elimination constant $\lambda_{z}: t_{1 / 2}=\ln 2 / \lambda_{z}$

- $\mathrm{MRT}$ (mean residence time): $\mathrm{MRT}=\mathrm{AUMC}_{0-\infty} / \mathrm{AUC}_{0-\infty}$, where $\mathrm{AUMC}$ is the area under the first moment curve, calculated by the trapezoidal rule and extrapolated to infinit

\section{Changes in pharmacokinetics of ASA metabolism:}

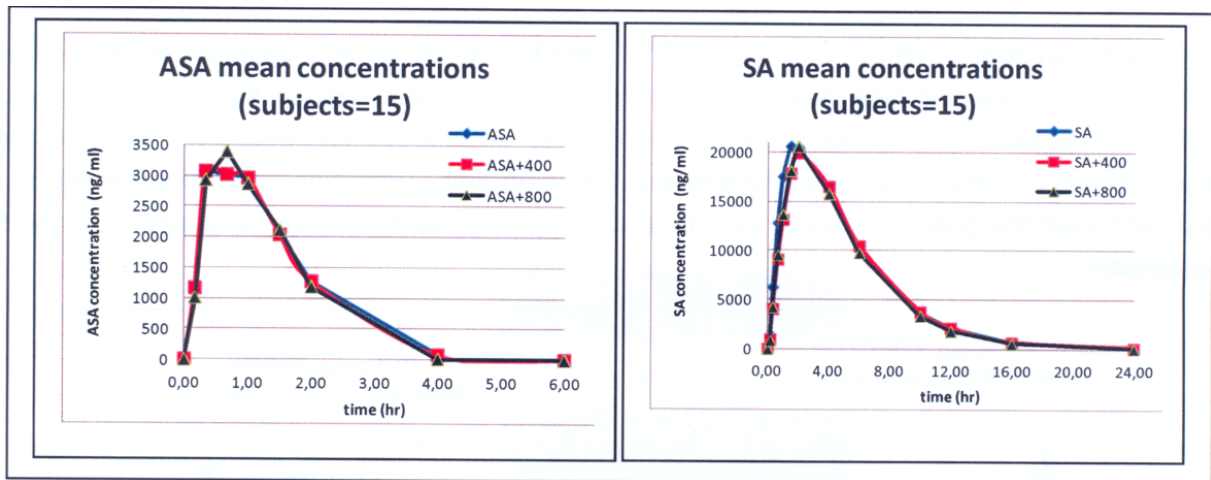

Figure 19. Mean concentration curves of aspirin (ASA)(left figure) and salicylic acid (SA)(right figure) plasma concentrations after oral administration of $500 \mathrm{mg} \mathrm{ASA}$ and co-administration of 400 and $800 \mu \mathrm{g}$ capsaicin tablets in 15 healthy male volunteers

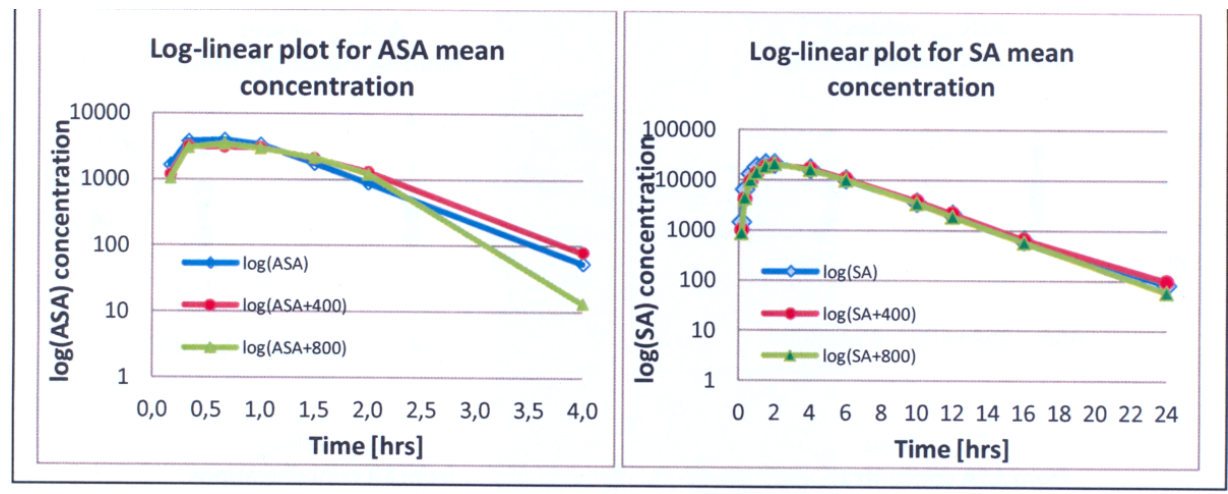

Figure 20. Log-linear plot curves of aspirin (ASA)(left figure) and salicylic acid (SA)(right figure) plasma concentrations after oral administration of $500 \mathrm{mg} \mathrm{ASA}$ and co-administration of 400 and $800 \mu \mathrm{g}$ capsaicin tablets in 15 healthy male volunteers 

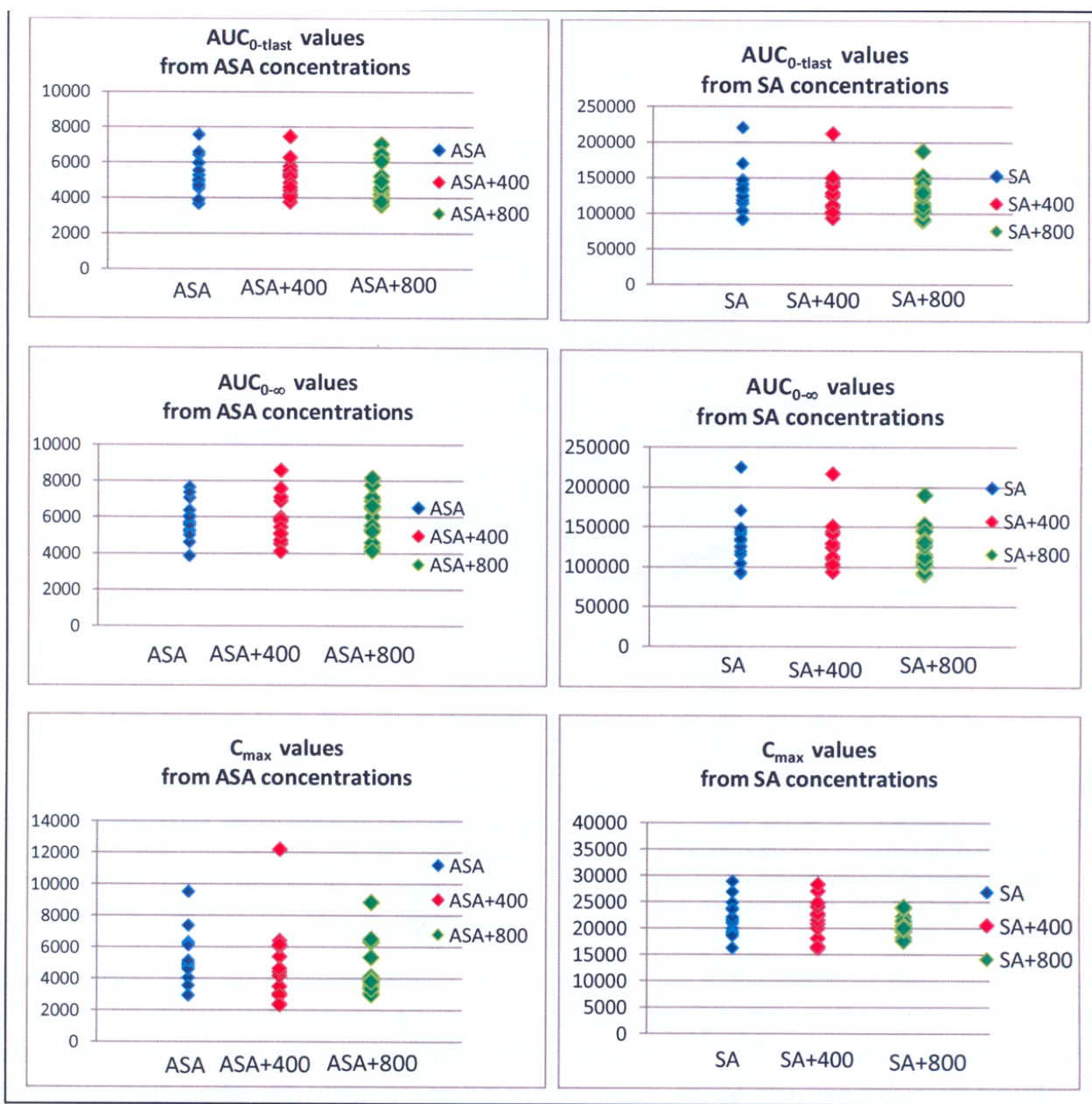

Figure 21. Changes of $A \cup C_{0 \text {-tlast }} A \cup C_{0-\infty}$ and $C_{\max }$ values for aspirin (ASA)(left figure) and salicylic acid (SA)(right figure) detected in plasma of 15 healthy male volunteers after oral administration of $500 \mathrm{mg}$ ASA and co-administration of 400 and $800 \mu \mathrm{g}$ capsaicin tablets 


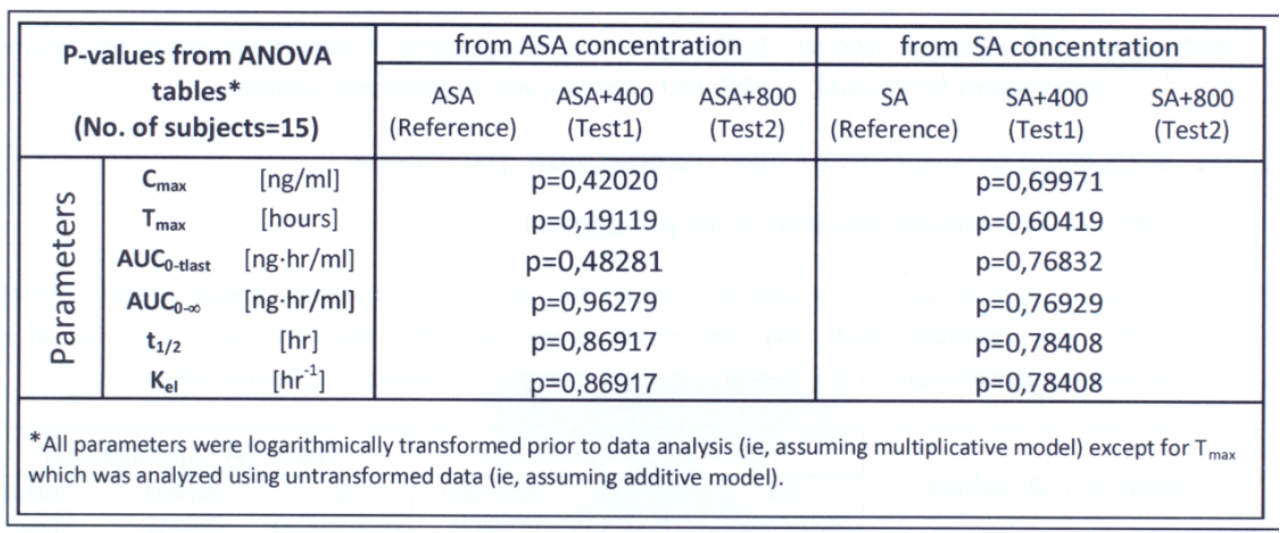

Table 18. $P$ values from NOVA table comparing the mean values of the PK parameters

\subsection{Platelet aggregation studies with ASA alone and in combination with capsaicin(oids)}

\subsubsection{Acetylsalicylic acid (ASA, Aspirin)}

- high doses (500 mg) decreases pain, fever and inflammation,

- continuously taken low doses $(75-325 \mathrm{mg}$ ) cause effective inhibition of platelet aggregation prevention of cardiovascular and cerebrovascular diseases,

- the incidence of coronary heart disease among ASA nonuser high cardiovascular risk patients is more than 15 cases per 1000 person-years,

- among low risk patients without ASA medication the incidence is less than 5 cases per 1000 person-years,

- high or low risk patients take low dose ASA everyday, the incidence of ACS became in the high risk group 4, in the low risk group only 1 event per 1000 patient-years.

\subsubsection{Upper gastrointestinal tract complications (UGIC) (peptic ulcer, bleeding, perforation)}

- among the general population 1 case / 1000 person-years,

- endpoints are $5-10 \%$,

- UGIC among patients on ASA is 2-3 cases / 1000 person-years,

- endpoints are $10-20 \%$,

- daily-users of low doses ASA have a 3-5 fold increased RR for UGIC as non-users.

\subsubsection{Study design}

- 15 healthy male volunteers, 
- prescreening procedure demographic data BMI, medical history, physical examination laboratory blood tests, urine test, urine drug test and viral serology (HBsAg, Anti HCV, HIV),

- subjects were 18-55 years old and had a BMI of $18-29.9 \mathrm{~kg} / \mathrm{m}^{2}$.

\section{Tablets}

Treatments

$400 \mu \mathrm{g}$ capsaicin $500 \mathrm{mg}$ ASA capsaicin placebo ASA placebo

\begin{tabular}{llll}
\hline $400 \mu \mathrm{g}$ capsaicin & 1 & 1 & 1 \\
\hline
\end{tabular}

$\begin{array}{lll}800 \mu \mathrm{g} \text { capsaicin } & 2 & 1\end{array}$

500 mg ASA $\quad 1 \quad 2$

\section{$400 \mu \mathrm{g}$ capsaicin + \\ $500 \mathrm{mg}$ ASA}

$800 \mu \mathrm{g}$ capsaicin +

500 mg ASA
2

1

Table 19. Treatment protocol for ASA induced platelet aggregation on the epinephrine-induced platelet aggreagtion

10.14.4. Study of capsaicinoids alone, ASA and their combinations ont he epinephrine-induced platelet aggregation int he healthy male volunteers.

Materials: following the drug administration (according to the randomized schedule of the permitted human phase I. protocol) $8.1 \mathrm{ml}$ blood was collected for platelet aggregation measurements before drug administration (0.0) (as control) and in 1.0, 2.0, 6.0 and 24.0 hours after drug administration.

Aggregation measurements: the epinephrine-induced platelet aggregation was studied by CARAT TX4 optical aggregometer (Carat Diagnostics Ltd, Budapest, Hungary at $37^{\circ} \mathrm{C}$ based on Born' method (Born, 1962; Koltai et al.,2008).

Method of evaluation of the obtained results: aggregation index below $40 \%$ was considered as clinically significant and effective inhibition of platelet aggregation.

Mathematical analysis of the obtained results was done by parired Student's t test (after using Kolmogonov-Smirnov' test to check the normality of the data distribution), one-way ANOVA test, Dunnett's post-hoc test. 


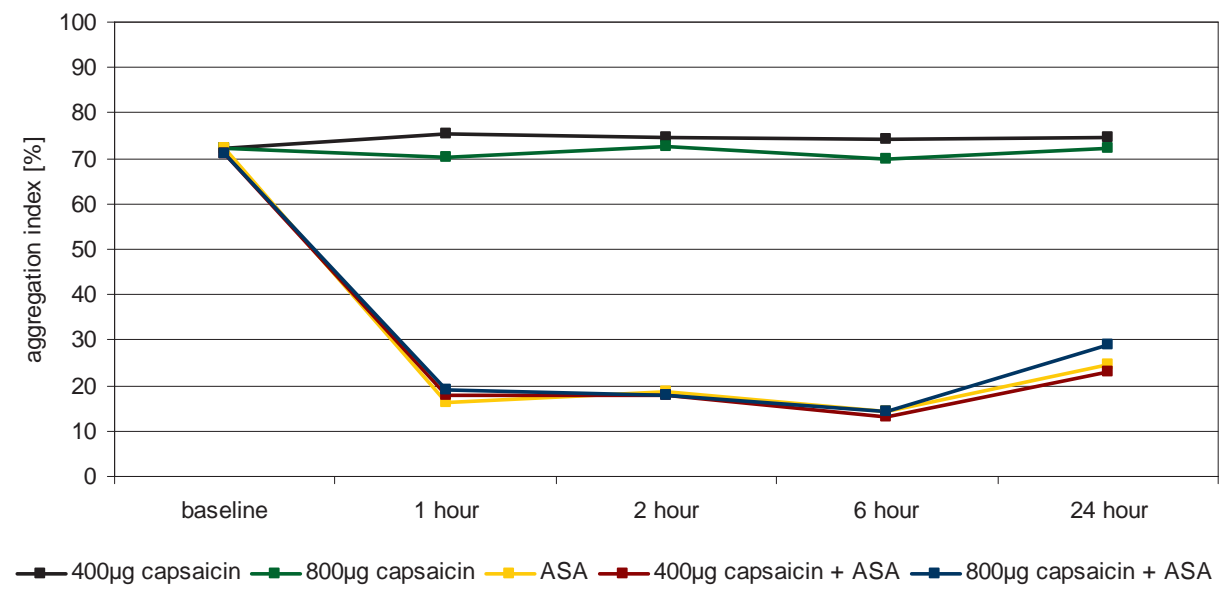

Figure 22. The platelet aggregation after oral capsaicin alone in two doses $(400,800 \mu \mathrm{g})$ and after co-administration of ASA (500 mg) in 15 male healthy subjects

\subsection{Summaries and conclusions}

- The human clinical pharmacological phase I. study (protocol number: 1.4.1; EudraCT number: 2008-007048-32) was successfully carried out in human healthy male volunteers.

- The capsaicin (oids) with using biodhesive compound was successfully pharmaceutically produced by PannonPharma Pharmaceutical Ltd. (Pécsvárad, Hungary

- The presence of capsaicin and dihydrocapsaicin (from the orally given capsaicinoids) was not able to detect in the plasma of healthy male volunteers, who were treated with capsaicin (oids) (in doses of 400 and $800 \mu$ g orally given).

- The capsaicin (oids) (given in doses of 400 and $800 \mu$ g orally does (do) not modify the absorption, metabolism and excretion of orally given ASA.

- The capsaicin (oids) does (do) not modify the epinephrine-induced aggregation by ASA, meanwhile the different doses of capsaicin(oids) alone have no direct action on the epinephrine-induced platelet aggregation.

- The capsaicin(oids) acts (act) locally in the gastrointestinal tract (indicating a good selection of bioadhesive compounds during the pharmaceutical technological preparation of our ASA +capsaicin(oids) combination.

- The results of these phase I. examinations offer us further possibility to carried out the forthcoming phase II. and phase III. examinations in patients. 


\section{Acknowledgements}

The study was supported by the grant of National Office for Research and Technology, "Pázmány Péter program" (RET-II 08/2005).

Authors express their thanks to physicians, medical nurses, assistants, technicians at First Department of Medicine and Institute of Cardiology, Medical and Health Centre, University of Pécs and chemists, techniciants at PannonPharma Pharmaceutical Ltd, Pécsvárad, Hungay for their excellent assistance.

This study was supported by the National Office for Research and Technology, "Pázmány Péter programme, RET-II 08/2005, by BAROS GÁBOR Programme, Hungary (REG_DKI_O, CAPSATAB) and by SROP-4.2.2.B-10/1/KONV-2010-029 (TAMOP 4.2.2./B).

\section{Author details}

Gyula Mózsik ${ }^{1}$, Tibor Past ${ }^{1}$, Tamás Habon ${ }^{1}$, Zsuzsanna Keszthelyi ${ }^{1}$, Pál Perjési², Mónika Kuzma², Barbara Sándor ${ }^{1}$, János Szolcsányi ${ }^{3}$, M.E. Abdel-Salam Omar ${ }^{4}$ and Mária Szalai ${ }^{5}$

*Address all correspondence to: gyula.mozsik@aok.pte.hu; gyula.mozsik@gmail.com 1 First Department of Medicine, Medical and Health Centre, University of Pécs, Hungary

2 Institute of Pharmaceutical Chemistry, Medical and Health Centre, University of Pécs, Hungary

3 Department ofPharmacology and Pharmacotherapy, Medical and Health Centre, University of Pécs, Hungary

4 Department of Pharmacology, National Research Centre, Dokki, Cairo, Egypt

5 PannonPharma Pharmaceutical Ltd., Pécsvárad, Hungary

The authors confirm that this overview content has no conflicts of interest.

\section{References}

[1] Abdel-Salam, O.M.E., Szolcsányi, J., Barthó, L., Mózsik, Gy. (1994): Sensory nervemediated mechanisms, gastric mucosal damage and its protection: A critical overview. Gastroprotection 2: 4-12 
[2] Abdel-Salam, O.M.E., Mózsik, Gy., Szolcsányi, J. (1995a): The effect of intragastrically administered capsaicin analogue in $\mathrm{HCl}$ and non- $\mathrm{HCl}$ dependent models of gastric mucosal injury. Z. Gastroenterol. 33; 80

[3] Abdel-Salam, O.M.E., Mózsik, Gy., Szolcsányi, J. (1995b): Capsaicin and its analogue Resiniferatoxin inhibit gastric acid secretion in pylorus-ligated rats. Pharmacol. Res. 31: $341-345$

[4] Abdel-Salam, O.M.E., Mózsik, Gy., Szolcsányi, J. (1995c): Effect of Resiniferatoxin on stimulated gastric acid secretory responses in the rat. J. Physiology (Paris) 88: 353-358

[5] Abdel-Salam, O.M.E., Mózsik, Gy., Szolcsányi, J. (1995d): Studies on the effect of intragastric capsaicin on gastric ulcer and on the prostacyclin-induced cytoprotection in rats. Pharmacol. Res. 32: 209-215

[6] Abdel-Salam, O.M.E., Bódis, B., Karádi, O., Szolcsányi, J., Mózsik, Gy. (1995e): Modification of aspirin and ethanol-induced mucosal damage in rats by intragastric application of Resiniferatoxin. Inflammopharmacology 3: 135-147

[7] Abdel-Salam, OME, Bódis, B., Karádi, O., Szolcsányi, J., Mózsik, Gy. (1995f): Nature of gastric $\mathrm{H}+$ back-diffusion approached by cimetidine, vagotomy, RTX and sucralfate. Ceská Slovenská Gastroenterologie. 49: 175-185

[8] Abdel-Salam, O.M.E., Bódis, B., Karádi, O., Nagy, L., Szolcsányi, J., Mózsik, Gy. (1995g): Stimulation of capsaicin-sensitive sensory peripheral nerves with topically applied Resiniferatoxin decreases salicylate-induced gastric $\mathrm{H}+$ back-diffusion in the rat. Inflammopharmacology 3: 121-133

[9] Abdel-Salam, O.M.E., Szolcsányi, J., Mózsik, Gy. (1996a): Differences in action of topical and system cystamine on gastric blood flow, gastric acid secretion and gastric ulceration in the rat. J. Physiology (Paris) 90:63-73

[10] Abdel-Salam, O.M.E., Szolcsányi, J., Porszász, R., Mózsik, Gy. (1996b): Effect of capsaicin and resiniferatoxin on gastrointestinal blood flow in rats. Eur. J. Pharmacol. 305: $127-136$

[11] Abdel-Salam, O.M.E., Mózsik, Gy., Szolcsányi, J. (1997a): The effect of capsaicin and Resiniferatoxin on the indomethacin-induced gastric mucosal damage in rats. In: Mózsik, Gy., Nagy, L., Király, Á. (eds.) (1997): Cell Injury and Protection in Gastrointestinal Tract. From Basic Science to Clinical Perspectives. Kluwer Academic Publisher, Dordrecht. pp.: 95-105

[12] Abdel-Salam, O.M.E., Mózsik, Gy., Szolcsányi, J. (1997b): The role of afferent sensory nerve in gastric mucosal protection. In: Mózsik, Gy., Nagy, L., Király, Á. (eds.): Twenty Five Years of Peptic Ulcer Research in Hungary. From Basic Science to Clinical Practice 1971-1995. Akadémiai Kiadó, Budapest, pp: 295-308

[13] Abdel-Salam, O.M.E., Szolcsányi, J., Mózsik, Gy., (1997c): The effect of Resiniferatoxin on experimental gastric ulcer in rats. In: Gaginella, T., Mózsik, Gy., Rainsford K.D. 
(eds): Biochemical Pharmacology as Approach to Gastrointestinal Disease. From Basic Science to Clinical Perspectives. Kluwer Academic Publisher, Dordrecht. pp.: 269-285

[14] Abdel-Salam, O.M.E., Szolcsányi, J., Mózsik, Gy., (1997d): The indomethacin-induced gastric mucosal damage in rats. Effect of gastric acid, acid inhibition, capsaicin-type agents and prostacyclin. J. Physiology (Paris) 97: 7-19

[15] Abdel Salam O.M.E., Sleem A.A., Hassan N.B., Sharaf H.A., Mózsik Gy. (2006): Capsaicin ameliorates hepatic injury caused by carbon tetrachloride in the rat. J. Pharmacol. Toxicol. 1:147-156.

[16] Abdel Salam, O. M. E., Czimmer, J., Debreceni, A., et al. (2001): Gastric mucosal integrity: Gastric mucosal blood flow and microcirculation. An overview, J. Physiol. 95, 105-127.

[17] Abdel Salam, O. M. E. Debreceni, A. and Mózsik, Gy. (1999). Capsaicin-sensitive afferent sensory nerves in modulating gastric mucosal defense against noxious agents, J. Physiol. 93, 443-454.

[18] Aijoka, H., Matsuura, N., Miyake, H. (2002): High quality of ulcer healing in rats by lafutidine and new-tipe histamine $\mathrm{H} 2$-receptor antagonist: involvement of capsaicin of sensitive sensory neurons. Inflammopharmacology 10, 483-493.

[19] Aijoka, H., Miyake, H., Matsuura, N. (2000): Effect of FRG-8813, a new-tipe histamine H2-receptor antagomist, on the recurrence of gastric ulcer healing by drug treatment. Pharmacology 61, 83-90.

[20] Alföldi, P., Obál, F. Jr., Tóth, E., Hideg, J. (1986): Capsaicin pretreatment reduces the gastric acid secretion elicited by histamine but does not affect the responses to carbachol and pentagastrin. Eur. J. Pharmacol. 123: 321-632

[21] Alföldi, P., Tóth, E., Obál, F., Hideg, J. (1987): Capsaicin treatment reduces histamineinduced gastric acid secretion in the rat. Acta Physiol. Hung. 69: 509-512

[22] Ames, B.N., McCann J., Yamasaki E. (1975): Methods for detecting carcinogens and mutagens with the Salmonella/microsoma test assay of 300 chemicals. Mutat Res. 31:347-364.

[23] Anonymus (1986): Metabolism and toxicity of capsaicin. Nutr. Rev. 44:20-22.

[24] Anu, A., Peter, K.V. (2000): The Chemistry of Paprika. Indian Species 37:15-18

[25] Ato, A., Yamamoto, M. (1996): Acute oral toxicity of capsaicin in mice and rats. J. Toxicol. Sci. 21:195-200.

[26] Azizan, A., Blevins, R.D. (1995): Mutagenecity and antimutagenecity testings of six chemicals associated with the purgent properties of specific spices as revelated by the Ames salmonella microsomal assay. Arch. Environ. Contam. Toxicol. 28:248-258. 
[27] Basu, S.K., De, A.K. (2003): Capsicum: Historical and Botanical Perspectives. Taylor and Francis Ltd., London. pp 1-15

[28] Berkesy, L. (1934): Effect of paprika on gastric secretion (in Hungarian). Orv. Hetil. Hung. Weekly Med. J. 78: 397-399

[29] Bevan, S., Szolcsányi, J. (1990): Sensory neuron-specific actions of capsaicin: mechanisms and application. Trends. Pharmacol. Sci. 11: 330-333

[30] Bevan, S., Yeats, J. (1990): Protons activate a cation conductance in a subpopulation of rat dorsal root ganglion neurons. J. Physiology (London) 433: 145-161

[31] Bley, K.R. (2004): Recent developments in transient receptor potential vanilloid 1 agonist-based therapies. Expert Opin Investig Drugs. 13:1445-1456.

[32] Boersch A., Calligham B.A., Lembeck F., Sharman D.F. (1991): Enzymatic oxidation of capsaicin. Biochem Pharmacol 41: 1863-1969.

[33] Born, CV. (1962): Aggregation of blood platelets by adenosine diphosphate and its reversal. Nature 194: 927-929.

[34] Boros, B., Dörnyei, Á., Felinger, A. (2008): Determination of capsaicin and dihydrocapsaicin in dog plasma by Liquid Chromatography -Mass Spectrometry (Analytical method report). PTE TTK Analitikai Kémiai Tanszék, Pécs, Hungary.

[35] Brash, AR., Baertschi, SW., Ingram, C.D., Harris, TM. (1988): Isolation and characterization of natural allene oxides: unstable intermediates in the metabolism of lipid hydroperoxides. Proc. Natl. Acad. Sci. USA 85: 3382-3386.

[36] Bruggeman, T.M., Wood, J.G., Davenport, H.W. (1979): Local control of blood flow in the dog's stomach: vasodilatation caused by acid back-diffusion following topical application of salicylic acid. Gastroenterology 77: 736-744.

[37] Buck, S.H., Miller, M.S., Burks, T.F. (1982): Depletion of primary afferent substance P by capsaicin and dihydrocapsaicin without altered thermal sensitivity. Brain Res. 233:216-220.

[38] Buck, S.H., Burks, T.F. (1986): The Neuropharmacology of capsaicin: review of some recent observation. Pharmacol. Rev. 38:179-226.

[39] Buiatti, E., Palli, D., Dacarli, A., Amaddori, D., Avelini, C., Biachi S., Biserni, S., Cipriani, E., Cocco, P., Giacoso, A., Marubini, E., Puntoni J., Blot, Jr. W. (1989): A casecontrol study of gastric cancer and diet in Italy. Int. J. Cancer 44:611-616.

[40] Cabanac, M., Cormareche-Leyder, M., Poirior, L.J. (1976): The effect of capsaicin on the temperature regulation of the rat. Flügers Arch. Ges. Physiol. 366: 217-221.

[41] Castle, N.A. (1992): Differential inhibition of potassium currents in rat ventricular myocytes by capsaicin. Cardivasc. Res. 26:1137-1144. 
[42] Caterina, M.J., Schumacker, M.A., Tomigana, M., Rosen, T.A., Levine, J.D., Julius, D. (1997): Capsaicin receptor: a heat activated ion channel in the pain pathyway. Nature 389:816-624.

[43] Chanda, S., Erexson, G., Riach, C., Innnes, D., Stevenson, F., Murli, H., Bley, K. (2004): Genotoxicity studies with pure trans-capsaicin. Mutat Res. 557:85-97.

[44] Chanda, S., Mould, A., Esmail, A., Bley, K. (2005): Toxicity studies with pure transcapsaicin delivered to dogs via intravenous administration. Reg. Toxicol. Pharmacol. 43:66-75.

[45] Chard, P.S., Bleakman, D., Saridge J.R. (1995): Capsaicin-induced neurotoxicity in cultured dorsal root ganglion neurons: involvement of calcium-activated proteases. Neuroscience 65: 1099-1108.

[46] Cheng, Y.P., Wang, Y.H. Cheng, L.P., He, R.R. (2003): Electrophysiologic effects of capsaicin on pacemaker cells in sinoatrial nodes of rabbits. Acta Pharmacol. Sin. 24:826-830.

[47] Chudapongse, P., Janthasoot, W. (1981): Mechanism of the inhibitory action on capsaicin on energy metabolism by rat liver mitochondria. Biochem. Pharmacol. 30: 735-740.

[48] Clive, D., Flamm, W.G., Machesko, M.R., Bernheim, N.J. (1972): A mutational assay system using the thymidine kinase locus in mouse lymphoma cells. Mutat Res. 16:77-87.

[49] Clive, D., Johnson, K.O., Spector, J.F., Batson, A.G., Brown, M.M. (1979): Validation and characterization of the L5178Y/TK+/- mouse lymphoma and mutagens assay system. Mutat Res. 59:61-108.

[50] Cordell, G.A., Araujo, O.D. (1993): Capsaicin: identification, nomenclature, and pharmacology. Ann. Pharmacother. 27:330-336.

[51] Couzin, J. (2004a): Withdrawal of Vioxx casts shadow over COX-1 inhibitors. Science 306: 3844-3850.

[52] Couzin, J. (2004b): Nail-biting time for trials of COX-2 drug. Science 306:1673-1675

[53] Csáky, T.Z. (1969): Introduction to general pharmacology. Appleton Century-Craft Educational Division, Meredith Corporation, New York, 17-34

[54] De, A.K., Ghosh, J. J. (1990): Inflammatory responses induced by substance P in rat paw. Indian J. Exp. Biol. 28: 946-948.

[55] De Lille, J., Ramirez, E. (1935): Pharmacodynamic action of the active principle of chilli (Capsicum annum). Chem. 4836 (abstract).

[56] Debreceni, A., Juricskay, I., Figler, M., Abdel-Salam O.M.E., Szolcsányi, J., Mózsik Gy. (1999): A direct stimulatory effect of small dose of capsaicin on gastric emptying 
rate in healthy human subjects measured by ${ }^{13} \mathrm{C}$ labeled octanoid acid breath test. J. Physiol. 93: 455-460.

[57] Desai, H.G., Venugopalam, K., Anita, F.P. (1973): Effect of red chili powder on NDA content of gastric aspirates. Gut 14: 974-976

[58] Donnerer, J., Lembeck, F. (1983): Capsaicin-induced reflex fall in rat blood pressure is mediated by afferent substances P-containing neurones via a reflex centre in the brain stem. Naunyn-Schmiedeber's Arch. Pharmacol. 324:293-295.

[59] Donnerer, J., Amann, R., Schuligoi, R, Lembeck, F. (1990): Absorption and metabolism of capsaicinoids following intragastric administration in rats. Naunin-Schmiedeberg's Arch Pharmacol. 342:357-361.

[60] Dugani, A.M., Galvin, G.B. (1986): Capsaicin effects on stress pathology and gastric acid secretion in rats. Life Sci. 39: 1531-1538.

[61] Dömötör. A., Szolcsányi, J., Mózsik, Gy. (2006): Capsaicin and glucose absorption and utilization in healthy human subjects. Eur. J. Pharmacol. 534: 208-283.

[62] Endoh, K., Leung, F.W. (1990): Topical capsaicin protects the distal but not the proximal colon against acetic acid injury. Gastroenterology 98: A 446.

[63] Espinosa-Aquire, JJ., Reyes, RE., Rubio, J., Ostrosky-Wegman, P., Martinez, G. (1993): Mutagenic activity of urban air samples and its modulation by chilli extracts. Mutat. Res. 303:55-61.

[64] Esplugues, J.V., Whittle, B.J.R., Moncanda, S. (1989): Local opioid-sensitive afferent sensory neurons in the modulation of gastric damage induced by PAF. Br. J. Pharmacol. 97: 579-585.

[65] Esplugues, J.V., Ramos, E.G, Gil, L., Esplugues, J. (1990): Influence of capsaicin-sensitive afferent neurons on the acid secretory responses of the rat stomach in vivo. Br. J. Pharmacol. 100: 491-496.

[66] Esplugues, J.V., Whittle, B.J.R. (1990): Morphine potentiation of ethanol-induced gastric mucosal damage in the rat. Role of local sensory afferent neurons. Gastroenterology 98: 82-89.

[67] Esplugues, J.V., Whittle, B.J.R., Moncanda, S. (1992): Modulation by opioids and by afferent sensory neurons of prostanoids protection of the rat gastric mucosa. Br. J. Pharmacol. 106: 846-852.

[68] Evangelista, S., Meli, A. (1989): Influence of capsaicin-sensory fibres on experimentally-induced colitis in rats. J. Pharm. Pharmacol. 41: 574-576.

[69] Evangelista, S., Santicioli, P., Maggi, C.A., Meli, A. (1989): Increase in gastric acid secretion induced by 2-deoxy-D-glucose is impaired in capsaicin pretreated rats. Br. J. Pharmacol. 98: 35-37. 
[70] Evans, H.J. (1962): Chromosomal aberrations produced by ionizing radiation. Int Rev Cytol 13:221-231.

[71] Evans, H.J. (1962): Cytological methods for detecting chemical mutagens. In: Hollander, A. (ed.) Chemical Mutagens, Principles and Methods for their Detection.Vol. 4. Plenum Press., New York and London, 1976. pp. 1-29.

[72] Expert Consensus Document on the Use of Antiplatelete Agents. ESC Eur. J. 2004.

[73] Fischer, G.A. (1958): Studies of the culture of leukemic cells in vitro. Ann. N. Y. Acad. Sci. 76:673-680.

[74] Flynn, D.L., Rafferty, M.F. (1986): Inhibition of human neutrophil 5-lipoxygenase activity by ginerdione, shogaol, capsaicin and related pungent compounds. Prostagland. Leucotrien. Med. 24:195-198.

[75] Freudenberg, K. (1962): Research on lignin. Fortschr. Chem. Org. Naturst. 20:41-72.

[76] Galloway, S.M., Aardema, M.J., Ischidate, M., et al. (1994): Report from working group on in vitro tests for chromosomal aberrations. Mutat Res. 312:241-261.

[77] Gislason, H., Guttu, K., Sorbye, H., Schifter, S., Waldum, L.H., Svanes, K. (1995): Role of histamine and calcitonin gene-related peptide in the hyperemic response to hypertonic saline and $\mathrm{H}^{+}$back-diffusion in the gastric mucosa of cats. Scand. J. Gastroenterol. 30: 300-310.

[78] Goso, C., Evangelista, S., Tramontana, N., Manzini, S., Blumberg, P.M., Szallasi, A. (1993): Topical capsaicin protects against trinitrobenzene sulfonic acid-induced colitis in the rat. Eur. J. Pharmacol. 249: 185-190.

[79] Govindarajan, V.S., Sathyanarayana, M.N. (1991): Capsicum-production, technology, chemistry, and quality. Part V. Impact on physiology, pharmacology, nutrition, and metabolism: structure pungency, pain, and desensitization sequences. Crit. Rev. Food Sci. Nutr. 29: 435-474.

[80] Glinsukon, T., Stitmunnaitnum, Y., Toskulkao, C., Buranawuth, T., Tandkrisanavinont, V. (1980): Acute toixicity of capsaicin in several animal species.Toxicon. $18: 215-220$.

[81] Gray, J.L., Bunnet, N.W., Orloff, S.L., Mulvihill, S.J., Debas, H.T. (1994): Role for calcitonin gene-related peptide in protection against gastric ulceration. Ann. Surg. 219: 58-64

[82] Green MD (2005). Causation, Vioxx and legal issues. Science 310: 973.

[83] Guengerich, FP., Kim, D.H., Iwasaki, M. (1991): Role of human cytochrome P-450 IIE1 in the oxidation of many low molecular weight cancer suspects. Chem. Res. Toxicol. 4: 168-179. 
[84] Gulbekian, S., Merighi, A., Wharton, J., Varndell, I.M., Polak, J.M, (1986): Ultrastructural evidence for the coexistence of calcitonin gene-related peptide and substance $\mathrm{P}$ in secretory vesicles of peripheral nerves in the guinea-pig. J. Neurocytol. 15: 535-542.

[85] Gunnett, P.M., Shi X., Lawson T., Kolar C., Toth B. (1997): Aryl radical formation during the metabolism of arylhydrazines by microsomes. Chem. Res. Toxicol. 10:1372-1377.

[86] Haim, N., Nemec, J., Roman, J., Sinha, BK. (1987a): In vitro metabolism of etoposide (VP-16-213) by liver microsomes and irreversible binding of reactive intermediates to microsomal proteins. Biochem. Pharmacol. 36: 527-536.

[87] Haim, N., Nemec, J., Roman, J., Sinha, BK. (1987b): Peroxidase-catalyzed metabolism of etoposide (VP-15-213) and covalent binding of reactive intermediates to cellular macromolecules. Cancer Res. 47: 5835-5840.

[88] Holzer, P., Sametz, W. (1986): Gastric mucosal protection against ulcerogenic factors in the rat mediated by capsaicin-sensitive afferent neurons. Gastroenterology 91: 975-981

[89] Holzer, P., Lippe, I.T. (1988): Stimulation of afferent nerve endings by intragastric capsaicin protects against ethanol-induced damage of gastric mucosa. Neuroscience 27: 981-987

[90] Holzer, P., Pabst, M.A., Lippe, I.T. (1989): Intragastric capsaicin protects against aspirin-induced lesion formation and bleeding in the rat gastric mucosa. Gastroenterology 96: 1425-1433.

[91] Holzer, P., (1990): Capsaicin-sensitive nerves in the control of vascular effector mechanisms. In: Green, B.G., Mason, J.R., Kare, M.R. (eds.): Chemical Senses. Irritation. MarcelDekker, New York. 2: 191-210.

[92] Holzer, P., (1991a): Capsaicin: cellular targets, mechanisms of action, and selectivity for thin sensory neurons. Pharmacol. Rev. 43: 144-202

[93] Holzer, P., (1991b): Afferent nerve-mediated control of gastric mucosal blood flow and protection In: Costa, M., Surrenti, C., Gorini, S., Maggi, C.A., Meli, A. (eds.): Sensory Nerve and Neuropeptides in Gastroenterology. From Basic Science to Clinical Perspective. Plenum Press, New York, pp.: 97-108.

[94] Holzer, P., (1992a): Capsaicin: selective toxicity for thin primary sensory neurons. Selective neurotoxicity. In: Herken, H., Hucho, F. (eds.): Handbook of Experimental Pharamacology. Springer Verlag, Berlin, pp.: 419-481.

[95] Holzer, P., (1992b): Peptidergic sensory neurons in the control of vascular functions: mechanisms and significance in the cutaneous and splanchnic vascular beds. Rev. Physiol. Biochem. Pharmacol. 121: 50-146. 
[96] Holzer, P., (1998): Neural emergency system in the stomach. Gastroenterology 114: 823-839

[97] Holzer, P. (1999). Capsaicin cellular targets, Mehanisms of action, as selectivity for thin sensory neurons, Phamacol. Res. 43. 143-201.

[98] Iwama, M., Tojima, T., Itol, Y., Takahashi N., Kanke, Y. (1990): Effects of capsaicin and ethanol on hepatic drug-metabolizing enzymes in rat. Int. J. Vit. Nutr. Res. 60:100-103.

[99] Jancsó, N., Jancsó-Gábor, A., (1959): Dauerausschaltung der Chemischen Schmerzempfindlichkeit durch capsaicin. Naunyn-Schmiedebergs Arch. Exp. Path. Pharmacol. 236: 142-145.

[100] Jancsó, N., Jancsó-Gábor, A., Szolcsányi, J. (1967): Direct evidence for neurogenic inflammation and its prevention by denervation and by pretreatment with capsaicin. Brit. J. Pharmacol. 31, 138-151.

[101] Jancsó, N., Jancsó- Gábor, A., Szolcsányi, J. (1968): The role of sensory nerves endings in the neurogen inflammation induced in human skin and in the eye and paw of the rat. Brit. J. Pharmacol. 33, 32-41.

[102] Jancsó-Gábor, A., Szolcsányi, J., Jancsó, N. (1970): Irrevesible impairement of the irregulation induced by capsaicin and similar pungent substances in rat and guineapigs. J. Physiol. (London) 206, 495-507.

[103] Jaiarj, P., Kitphati, C., Sinchaipanid, N., Lertsin, N., Siripanyachan, P. (2000): Stability testing of capsaicinoid cream, Mahidol University Annual Research Abstracts 371

[104] Joe, B., Lokesh, B R. (1994): Role of capsaicin, curcumin and dietary n-3 fatty acids in lowering the generation of reactive oxygen species in rat peritoneal macrophages. Biochim. Biophys. Acta 1224:255-263.

[105] Jurenitsch, J., Kubelka, W., Jentzsch, K. (1979): Identification of cultivated taxa of Capsicum. Taxonomy, anatomy and composition of purgent principles. Plant. Med. 35: 175-183.

[106] Karádi, O., Mózsik, Gy. (2000). Surgical and chemical vagotomy on the gastrointestinal mucosal defense. Akadémiai Kiadó, Budapest.

[107] Kawada, T., Suzuki, T., Takahashi, M., Iwai K. (1984): Gastrointestinal absorption and metabolism of capsaicin and dihydrocapsaicin in rats. Toxicol. Appl. Pharmacol. 72:449-456.

[108] Kawada, T., Iwai, K. (1985): In vivo and in vitro metabolism of dihydrocapsaicin, a pungent principle of hot pepper, in rat. Agric. Biol. Chem. 49:441-448.

[109] Kawai, S., Nishida, S., Kato, M., Furumaya, Y., Okomoto, T., Mizushima Y. (1998): Comparison of cyclooxigenase- 1 and-2 inhibitory activities of nonsteroidal anti-in- 
flammatory drugs using humaín platelets and synovial cells. Eur. J. Pharmacol. 347:87-94.

[110] Kensler, T.W., Egner, PA, Davidson, NE., Roebuck, BD, Pikul, A, Groopman, JD. (1986): Modulation of aflatoxin metabolism, aflatoxin-N7-guanine formation, and hepatic tumorigenesis in rats fed ethoxyquin: role of induction of glutathione S-transferases. Cancer Res. 46: 3924-3931.

[111] Ketusinh, O., Dhorranintra, B., Juengjareon, K. (1966): Influence of capsaicin solution on gastric acidities. Am. J. Protocol. 17: 511-515.

[112] Koop, DR. (1992): Oxidative and reductive metabolism by cytochrome P450 2E1. FASEB J. 6: 724-730.

[113] Koltai, K., Fehér G., Kenyeres, P., Lenard, I., Alexy, T., Horváth, B., Márton, Z., Késmárky, G., Tóth K. (2008): Relation of platelet aggregation and fibrinogen levels to advancing age in aspirin- and thioenopyrimide-treated patients. Clin. Hemorheol. Microcirc. 40: 295-302.

[114] Kopec, S.E., DeBellis, R.J., Irwin, R.S. (2002): Chemical Analysis of Freshly Prepared and Stored Capsaicin Solutions: Implications for Tussigenic Challenges. Pulm. Ther. 15: 529-534.

[115] Lawler, A. (2005): The law.Vioxx verdict: too little or too much science? Science 309:1481.

[116] Lawson, T., Gannett, P. (1989): The mutagenicity of capsaicin and dihydrocapsaicin in V79 cells. Cancer Lett. 49:109-113.

[117] Lee, S.S., Kumar, S. (1980): Microsomes, Drug Oxidations, and Chemical Calcinogenesis. Vol.2. In: Coon, M.J., Conney, A.H., Estabrook, R.W., Gelboin, H.V., Gillette, J.R., O’ Brien, P.J. (eds.): Academic Press, New York, pp. 1009-1012.

[118] Lenzer, J, (2004): FDA is incapable of protecting of US “against another Vioxx". Brit. Med. J. 329:1253.

[119] Li, D.S., Raybould, H.E., Quintero, E., Guth, P.H. (1992): Calcitonin gene-related peptide mediates the gastric hyperemic response to acid back-diffusion. Gastroenterology 102: 1124-1128.

[120] Lippe, I.T., Pabst, M.A., Holzer, P. (1989): Intragastric capsaicin enhances rat gastric acid elimination and mucosal blood flow by afferent nerve stimulation. Br. J. Pharmacol. 96: 91-100.

[121] Lippe, I.T., Holzer, P. (1992): Participation of endothelium-derived nitric oxide but not prostacyclin in the gastric mucosal hypeaemic response due to acid back-diffusion. Br. J. Pharmacol. 105: 708-714.

[122] Maga, J.A., (1975): Capsicum. Crit. Rev. Food. Sci. Nutr. 177-199. 
[123] Maggi, C.A., Meli, A. (1988): The sensory-efferent function of capsaicin-sensitive sensory neurons. Gen. Pharmacol. 19: 1-43.

[124] Maggi, C.A., Santicioli, P., Geppetti, P., Parlani, M., Astolfi, M., DelBianco, E., Patacchini, R., Giuliani, S., Meli, A., (1989): The effect of calcium free medium and nefidipine on the release of substance P-like immunoreactivity and contractions induced by capsaicin in the isolated guinea-pig bladder. Gen. Pharmacol. 40: 445-456.

[125] Maggi, C.A., (1995): Tachykinins and calcitonin gene-related peptide (CGRP) as cotransmitters released from peripheral endings of sensory nerves. Progress in Neurobiology 45: 1-98.

[126] Makara, G.B., Frenkl, C.R., Somfai, Z., Szepesházi, K. (1965): Effect of capsaicin on the experimental ulcer in the rat. Acta. Med. Sci. Hung. 21: 213-216.

[127] Mans, D.R., Lafleur, M.V., Westmijze E.J., van Maanen, J.M., van Schaik, M.A., Lankelma, J., Retel, J. (1991): Formation of different reaction products with single-and double-stranded DNA by the ortho-quinone and the semi-quinone free radical of etoposide (VP-16-213). Biochem. Pharmacol. 42: 2131-2139.

[128] Maron, D.M., Ames, B.N. (1983): Revized methods for the Salmonella mutagenecity test. Mutat Res. 113:173-215.

[129] Marques, S., Oliveira, N.G., Chaveca, T., Rueff, J. (2002): Micronuclei and sister chromatid exchanges induced by capsaicin in human lymphocytes. Mutat Res. 517:39-46.

[130] Martin, R.L., McDermott, S.J., Salmen, H.J., Palmatier, J., Cox, B.F., Gintant, G.A. (2004): The utility of hERG and repolarization assays in evaluating delayed cardiac reporalization: influence of multi-channel block. J. Cardiovasc. Pharmacol. 43:369-379.

[131] Mcgettigan, P., Henry, D (2006): Cardiovascular Risk and Inhibition of Cyclooxygenase. A System Review of the Observational Studies of Selective and Nonselective Inhibitors of Cyclooxygenase 2 JAMA 2006; 296.

[132] Merighi, A., Polak, J.M., Gibson, S.J., Gulbekian, S., Valentino, K.L., Peirone S.M., (1988): Ultrastructural studies on calcitonin gene-related peptide-, tachykinin- and somatostatin-immunoreactive neurons in rat dorsal root ganglia: Evidence for colocalization of different peptides in single secretory granules. Cell Tiss. Res. 254: 101-109.

[133] Miller, M.S., Brendel, K., Burks, T.F., Sipes, I.G. (1983): Interaction of capsaicinoids with drug-metabolizing systems. Relationship to toxicity. Biochem. Pharmacol. 32:547-551.

[134] Modly, C.E., Das, M., Don, P.S., Marcelo C.L., Mukhtar, H., Bickers, D.R. (1986): Capsaicin as an in vitro inhibitor of benzo(a)pyrene metabolism and its DNA binding in human and murine keratinocytes. Drug. Metab. Dispos. 14: 413-416. 
[135] Molnar, J.(1965): Die pharmakologischen wirkungen des capsaicin, des schaf schmeckenden wirkstoffers im paprika. Arzeilmittel-Forsh. L5: 718.

[136] Molnar, J., György, L. (1967): Pulmonary hypertensive and other haemodynamic effect of capsaicin in the cat. Eur. J. Pharmacol.1: 86-92.

[137] Monsereenusorn, Y. (2001): Subchronic toxicity studies of capsaicin and capsinum in rats. Res. Commmun. Chem. Path. Pharmacol. 41:95.110.

[138] Moore. M.M., Honna, M., Clements, J., et al. (2003): Mouse thymidine kinase gene mutation assay: International workshop on genotocicity test. Workgroup Report. Plymouth, UK. 2002. Mutat Res. 540:127-140.

[139] Mózsik, Gy., Moron, F., Jávor, T. (1982): Cellular mechanisms of the development of gastric mucosal damage and of gastroprotection induced by prostacyclin in rats. A pharmacological study, Prostagland. Leukot. Med. 9, 71-84.

[140] Mózsik, Gy., Király, Á., Sütö, G., Vincze, Á. (1993): ATP breakdown and resynthesis in the development of gastrointestinal mucosal damage and its prevention in animals and human (an overview of 25 years ulcer research studies). In: Mózsik, Gy., Pár, A., Kitajima, M., Kondo, M., Pfeiffer, CJ., Rainsford, KD., Sikiric, P., Szabó, S. (eds.): Cell Injury and Protection in the Gastrointestinal Tract: From Basic Science to Clinical Perspectives. Akadémiai Kiadó, Budapest, pp.: 39-80.

[141] Mózsik, Gy., Abdel-Salam, O.M.E., Bódis, B., Karádi, O., Nagy, L., Szolcsányi, J. (1996a): Role of vagal nerve in defense mechanisms against NSAIDs-induced gastrointestinal mucosal damage. Inflammopharmacology 4: 151-172.

[142] Mózsik, Gy., Abdel-Salam, O.M.E., Bódis, B., Karádi, O., Király, Á., Sütö, G., Rumi, Gy., Szabó, I., Vincze, Á. (1996b): Gastric mucosal protective effects of prostacyclin and $\beta$-carotene, and their biochemical backgrounds in rats treated with ethanol and $\mathrm{HCl}$ in dependence of their doses and of their time after administration of necritizing agents. Inflammopharmacology 4: 361-378.

[143] Mózsik, Gy., Nagy, L., Király, Á. (eds.) (1997a): Twenty Five Years of Peptic Ulcer Research in Hungary. From Basic Science to Clinical Practice 1971-1995. Akadémiai Kiadó, Budapest, pp.: 1-448.

[144] Mózsik, Gy., Nagy, L., Pár, A., Rainsford, K.D. (eds.) (1997b): Cell Injury and Protection in the Gastrointestinal Tract: From Basic Science to Clinical Perspectives. Kluwer Academic Publisher, Boston, Dordrecht.

[145] Mózsik, Gy., Abdel-Salam, O.M.E., Szolcsányi, J. (1997c): Capsaicin-Sensitive Afferent Nerves in Gastric Mucosal Damage and Protection. Akadémiai Kiadó, Budapest.

[146] Mózsik, Gy., Debreceni, A., Abdel-Salam, OME., Szabó, I., Figler, M., Ludány, A., Juricskay, I., Szolcsányi, J. (1999): Small doses capsaicin given intragastrically inhibit gastric secretion in healthy human subjects. J. Physiol. Paris 93: 433-436 
[147] Mózsik, Gy., Vincze, Á., Szolcsányi, J. (2001): Four responses of capsaicin sensitive primary afferent neurons to capsaicin and its analog. Gastric acid secretion, gastric mucosal damage and protection. J. Gastroenterol. Hepatol. 16, 193-197.

[148] Mózsik, Gy., Belágyi, J., Szolcsányi, J. (2004a): Capsaicin-sensitive afferent nerves and gastric mucosal protection in the human healthy subjects. A critical overview. in: Takeuchi K., Mózsik Gy. (eds.) Research Signpost Kerala, India pp. 43-62.

[149] Mózsik, Gy., Pár, A., Pár, G. et al. (2004b). Insight into the molecular pharmacology to drugs acting on the afferent and efferent fibres of vagal nerve in the gastric mucosal protection in: Ulcer Research, Proceedings of the 11th International Conference, Sikirič P., Seiwerth P., Mózsik Gy., Arakawa T., Takeuchi K. (eds.): pp. 163-168. Monduzzi, Bologna.

[150] Mózsik, Gy., Rácz, I., Szolcsányi, J. (2005): Gastroprotection induced by capsaicin in healthy human subjects. World J. Gastroenterol. 11:5180-5184.

[151] Mózsik Gy.(2006a).Molecular pharmacology and biochemistry of gastroduodenal mucosal damage and protection. In: Mózsik, Gy. (ed.) Discoveries in Gastroenterology: from Basic Research to the Clinical Perspectives.Akadémiai Kiadó, Budapest, pp 139-224.

[152] Mózsik Gy., Dömötör, A., Abdel-Salam O.M.E. (2006b): Molecular pharmacological approach to drug actions on the afferent and efferent fibres of the vagal nerve in the gastric mucosal protection in rats. Inflammopharmacology 14: 243-249.

[153] Mózsik, Gy., Szolcsányi, J., Dömötör, A. (2007a): Capsaicin research as a new tool to approach of the human gastrointestinal physiology, pathology and pharmacology. Inflammopharmacology 15: 232-245.

[154] Mózsik Gy., Past T.,Perjési P. (2007b): Capsaicinoids,nonsteroidal antiinflammatory drugs and gastrointestinal protection. An expert' opinion. Pécs, Hungary.

[155] Mózsik, Gy., Past, T., Perjési, P., Szolcsányi, J. (2008a): Original Reports on Toxicology of Capsaicin I. The Testing of Capsaicin Natural USP 27 with Bacterial Reverse Mutation Tests. The date of final report 13 September 2007. Study code: 07/496-007M. Veszprém, LAB International Research Centre Hungary Ltd. 2008; 1-33 (7 appendix).

[156] Mózsik, Gy., Past, T., Perjési, P., Szolcsányi J. (2008b): Original Reports on Toxicology of Capsaicin II. Testing of Mutagenic Effect of Test Item Capsaicin Natural USP 27 by Mouse Micronucleus Test. The date of final report 12 October 2007. Study Code: 07/019-008E. Budapest, Toxic-Coop Ltd. 2008; 1-18 (5 appendix).

[157] Mózsik, Gy., Past, T., Perjési, P., Szolcsányi, J. (2008c): Original Reports on Toxicology of Capsaicin III. 14-Day Oral Gavage Dose Range Finding Study with Capsaicin Natural USP 27 in the Rats. The date of final report 15 October 2007. Study Code: 07/018-100PE. Dunakeszi, Toxic-Coop Ltd. 2008; This report consists of 27 pages of text, 51 pages of appendices. 
[158] Mózsik, Gy., Past, T., Perjési, P., Szolcsányi, J. (2008d): Original Reports on Toxicology of Capsaicin IV. Oral Dose Range Finding Toxicity Study in Beagle Dogs with Capsaicin Natural USP 27. The date of final report 08 October 2007. Study Code: 07/496-100KE. Veszprém, LAB International Research Centre Hungary Ltd. 2008; 1-26 (17 appendix)

[159] Mózsik, Gy., Past T., Perjési, P., Szolcsányi, J. (2008d): Original Reports on Toxicology of Capsaicin V. Oral Dose Range Finding Toxicity Study of Capsaicin Natural USP 27 in Beagle Dogs (Supplementary Final Report). The date of final report 08 October 2007. Study Code: 07/496-100KE. Veszprém, LAB International Research Centre Hungary Ltd. 2008; 1-20 (10 appendix)

[160] Mózsik Gy, Past T, Perjési P, Szolcsányi J. (2008e): Original Reports on Toxicology of Capsaicin VI. 28-Day Oral Toxicity Study of Capsaicin Natural USP 27 in Rats (Final Report). The date of final report 21 May 2008. Study Code: 07/018-100P. Dunakeszi Toxic-Coop Ltd. 2008; This report consists of 31 pages text and 166 pages of appendix.

[161] Mózsik, Gy., Past, T., Perjési, P., Szolcsányi J. (2008f): Original Reports on Toxicology of Capsaicin VII. 8-Day Oral Toxicity Study of Test Item Capsaicin Natural USP 27 in Beagle Dogs (Final Report). The date of final report 13 June 2008. Study Code: 07/496-100K. Veszprém, LAB International Research Centre Hungary Ltd. 2008; 1-35 (90 appendix).

[162] Mózsik, Gy., Past, T., Perjési, P., Szolcsányi, J. (2008g): Original Reports on Toxicology of Capsaicin VIII. Amendment 2 to Study Plan. 28-Day Oral Toxicity Study of Test Item Capsaicin Natural USP 27 in Beagle Dogs. The date of final report 03 June 2008. Study Code: 07/496-100K. Veszprém, LAB International Research Centre Hungary Ltd. 2008; $1-4$.

[163] Mózsik, Gy., Past, T., Perjési, P., Szolcsányi, J. (2008h): Determination of capsaicin and dihydrocapsaicin content of dog's plasma by HPLC-FLD method. In.: Mózsik Gy., Past T., Perjési P., Szolcsányi J.: Original Reports on Toxicology of Capsaicin VII. 8-Day Oral Toxicity Study of Test Item Capsaicin Natural USP 27 in Beagle Dogs (Final Report). The date of final report 13 June 2008. Study Code: 07/496-100K LAB. Veszprém, International Research Centre Hungary Ltd. 2008; 1-35 (190 appendix).

[164] Mózsik, Gy., Past, T., Abdel Salam, O.M.E, Kuzma, M., Perjési, P. (2009a): Interdisciplinary review for the correlation between the plant origin capsaicinoids, non-steroidal antiinflammatory drugs, gastrointestinal mucosal damage and prevention in animals and human beings. Inflammopharmacology 17: 113-150.

[165] Mózsik, Gy., Dömötör, A., Past, T., Vas, V., Perjési, P., Kuzma, M., Blazics, Gy., Szolcsányi, J. (2009b): Capsaicinoids: From the plant cultivation to the production of the human medical drug. Akadémiai Kiadó, Budapest. 
[166] Mózsik, Gy., Past, T., Dömötör, A., Kuzma, M., Perjési P. (2010): Production of orally applicable new drug or drug combinations from natural origin capsaicinoids for human medical therapy. Curr. Pharm. Des. 16: 1197-1208.

[167] Nagabhuslan, M., Blide, S.V (1985): Mutagenecity of chili extract and capsaicin in short term tests. Environ. Mutagen 7: 881-888.

[168] Nopanitaya, W. (1974): Effects of capsaicin in combination with diets of varying protein content on the duodenal absorptive cells of the rat. Am. J. Dig. Dis. 19: 439-449.

[169] Notani, P.N., Jayant, K. (1987): Role of diet in upper aerodigestive tract cancers. Nutr. Cancer. 10:203-113.

[170] Newmark H.L. (1984): A hypothesis for dietary componets as blocking agents of chemical carcinogenesis: plant phenolics and pyrole pigments. Nutr.Cancer 6:58-70.

[171] Newmark H.L. (1987): Plant phenolics as inhibitors of mutational and precarcinogenic events. Can J Physiol Pharmacol 65:461-466.

[172] Oi, Y., Kawada ,T., Watanabe, T., Iwai, K. (1992): Induction of capsaicin- hydrolyzing enzyme activity in rat liver by continuous oral administration of capsaicin. J. Agric. Food Chem. 40:467-470.

[173] Onodera, S., Shibata, M., Tanaka. H, et al. (1999): Gastroprotective mechanisms of lafutidine, a novel anti-ulcer drug with histamine $\mathrm{H}_{2}$-receptor antagonist activity, Artneim. Forsch., Drug. Res. 49, 519-526.

[174] Onodera, S., Shibata, M., Tanaka, H., et al. (2002). Gastroprotective activity of FRG-8813, a novel histamine $\mathrm{H}_{2}$-receptor antagonist, in rats, Jpn. J. Pharmacol. 68, 161-173.

[175] Opez-Carrillo, L., Lopez-Cervantes, M., Bobles-Diaz, G., Ramilez-Espitia, A., MoharBetancourt, A., Menses-Gratia, A., Lopez-Vidal, Y., Blair A. (2003): Capsaicin consumption Helicobacter pylori positivity and gastric cancer in Mexico. Int. J. Cancer 106:277-282.

[176] Patrono, C., Bachmann, F., Baigent, C., Bode, C., De Caterina, R, Charbonnier, B., et al. (2004): Expert consensus document on the use of antiplatelet agents. The task force on the use of antiplatelet agents in patients with atherosclerotic cardiovascular disease of the Europen Society of Cardiology. Eur. Heart J. 25: 166-181.

[177] Pabst, M.A., Schöninkle, E., Holzer, P. (1993): Ablation of capsaicin-sensitive afferent neurons impairs defense but not rapid repair of rat gastric mucosa. Gut 34: 897-903.

[178] Park, Y.H., Lee, S.S. (1994): Identification and characterization of capsaicin-hydrolyzing enzymes purified from rat liver microsomes. Biochem. Mol. International 34:351-360. 
[179] Pique, J.M., Esplugues, J.V., Whittle, B.J.R. (1990): Influence of morphine or capsaicin pretreatment on rat gastric microcirculatory response to PAF. Am. J. Physiol. 258: G352-357.

[180] Rauf, M., Bachmann, E., Metwally, S.A. (1985): J. Drug. Res. Egypt 16:29-36.

[181] Raybould, H.E., Taché, Y. (1989): Capsaicin-sensitive vagal afferent fibres and stimulation of gastric acid secretion in anesthetized rats. Eur. J. Pharmacol. 167: 237-243.

[182] Reilly, C.A., Crouch, D.J., Yost, G.S., Fatah, A.A. (2002): Determination of capsaicin, noivamide and dihydrocapsaicin in blood and tissue by liquid chromatography-tandem mass spectrometry. J. Anal. Toxicol. 26: 313-319.

[183] Reinshage, M., Pate, A., Sottili, M., Nast, C., Davis, W., Muellr, K., Eysselein, V.E. (1994): Protective functions of extrinsic sensory neurons in acute rabbit experimental colitis. Gastroenterology 106: 1208-1214.

[184] Richeux, F., Cascante, M., Ennamary, F., Sabourcau, D., Creppy, E.E. (1999): Cytotoxicity and genotoxicity of capsaicin in human neuroblastoma cells SHSY-5Y. Arch. Toxicol. 73:403-409.

[185] Ritchie, W.P. Jr. (1991): Mediators of bile acid induced alterations in gastric mucosal blood flow. Am. J. Surg. 161: 126-129.

[186] Robert, A., Olafsson, A.S., Lancaster, C., Zhang, W. (1991): Effects of capsaicin and of capsaicin denervation on gastric secretion and gastric lesions produced by ulcerogenic agents. Exp. Clin. Gastroenterol. 1: 5 (abst.).

[187] Rozin, P. (1990): Getting to like the burn of chili pepper. Biological, physiological, and cultural perspective. In: Green, B.G., Manson, J.R., Kare, M.R. (eds.): Chemical Senses. Irritation. Vol 2. Marcel Dekker, New York, pp.: 231-269.

[188] Saito, A., Yamamoto, M. (1996): Acute oral toxicity of capsaicin in mice and rats. J. Toxicol. Sci. 21: 195-200.

[189] Sarlós, P., Rumi, Gy., Szolcsányi, J., Mózsik, Gy., Vincze, Á. (2003): Capsaicin prevents the indomethacin-induced gastric mucosal damage $\mathrm{n}$ human healthy subject. Gastroenterology 124, Suppl. 1, A-511.

[190] Savitha, G., Panchanathan, S, Salimath, B.P. (1990): Capsaicin inhibits calmodulinmediated oxidative burst in rat macrophages. Cell Signal. 2: 577-585.

[191] Schneider, M.A., de Luca, V., Gray, S.J. (1956): The effect of spice ingestion upon the stomach. Am. J. Gastroenterlol. 26: 722-732.

[192] Schweiggert, U., Schieber A., Carle R. (2006): Effects of blanching and storage on capsaicinoid stability and peroxidase activity of hot chili peppers. Innovative Food Science and Emerging Technologies. 
[193] Solanke, T.F. (1973): The effect of red pepper (Capsicum frutescens) on gastric acid secretion. J. Surg. Res. 15: 385-390.

[194] Starlinger, M., Schiessel, R., Hung, C.R. (1981a): H+ back-diffusion stimulating mucosal blood flow in the rabbit fundus. Surgery 89: 232-236.

[195] Surh, Y.J., Ahn, S.H., Kim, K.C., Park, J.B., Sohn, Y.W., Lee, S.S. (1995): Metabolism of capsaicinoids: evidence for alipharic hydroxylation and its pharmacological implications. Life Sci. 56, pp 305-311.

[196] Surh, Y.J., Lee, S.S (1995): Capsaicin a double-edged swort: toxicity, metabolism, and chemopreventive potential. Life Sci. 56:1845-1855.

[197] Szállasi, A., Blumberg, M. (1999): Vanilloid (capsaicin) receptors and mechanisms. Pharmacol. Rev. 51, 159-211.

[198] Szabo, IL., Czimmer, J., Szolcsányi, J., Mozsik, Gy. (2013): Molecular pharmacological approaches to effects of capsaicinoids and of classical antisecretory drugs on gastric basal acid secretion and on indomethecin-induced gastric mucosal damage in human healthy subjects (Mini review) Curr. Pharm. Des. 19: 84-89.

[199] Szolcsányi, J., (1977): A pharmacological approach to elucidation of the role of different nerve fibres and receptor endings in mediation of pain. J. Physiology (Paris) 73: 251-259.

[200] Szolcsányi, J., Barthó, L. (1981): Impaired defense mechanisms to peptic ulcer in the capsaicin-desensitized rat. In: Mózsik, Gy., Hänninen, O., Jávor, T. (eds.): Advances in Physiological Sciences. Vol. 29. Gastrointestinal Defense Mechanisms. Pergamon Press and Akadémiai Kiadó, Oxford and Budapest, pp.: 39-51.

[201] Szolcsányi, J., (1982): Capsaicin type pungent agents producing pyrexia. In: Milton, A.S. (ed.): Handbook of Experimental Pharmacology. Vol. 60. Pyretics and Antipyretics. Springer-Verlag, Berlin pp.: 437-478.

[202] Szolcsányi, J., (1984): Capsaicin-sensitive chemoceptive neural system with dual sensory-efferent function. In: Chahl, L.A., Szolcsányi, J., Lembech F. (eds.): Antidromic Vasodilatation and Neurogenic Inflammation. Akadémiai Kiadó, Budapest, pp.: 27-56

[203] Szolcsányi, J., (1985): Sensory receptors and the antinociceptive effect of capsaicin. In: Hakanson, R., Sundler, F. (eds.): Tachykinin Antagonists. Elsevier, Amsterdam, 45-54

[204] Szolcsányi, J. (1990a): Capsaicin, irritation, and desenzitation: Neurophysiological and future perspectives. In: Vo. 2. Green, B.G., Mason, J.R., Kare, M.R. (eds.): Chemical Senses. Irritation. Marcel Dekker, New York, pp.: 141-169.

[205] Szolcsányi, J. (1990b): Effect of capsaicin, rediniferatoxin and piperine on ethanol-induced gastric ulcer of the rat. Acta Physiol. Hung. 75: 267-268 
[206] Szolcsányi, J. (1993): Actions of capsaicin on sensory receptors. In: Wood, J.N. (ed.): Capsaicin in the Study of Pain. Academic Press, London, pp.: 1-33.

[207] Szolcsányi, J., Pórszász, R., Pethő, G., (1994): Capsaicin and pharmacology of nociceptors. In: Besson, J.M., Besson, G., Ollat, H. (eds.): Peripheral Neurons in Nociception. Physicopharmacological Aspects, Paris, John Libby Eurotext. pp.: 109-124.

[208] Szolcsányi, J., (1996): Capsaicin-sensitive sensory nerve terminals with local and systemic efferent functions: facts and scopes of unorthodox neuroregulatory mechanisms. In: Kumazawa, T., Kumazawa, L., Mizumura, K. (eds.): The Polymodal Receptor - A Gateway to Pathological Pain. Progress in Brain Research. Vo.113. Elsevier, Amsterdam, pp.: 343-359.

[209] Szolcsányi, J. (1997): A pharmacological approach to elucidation of the role of different nerve fibres and receptor endings inmediation of pain. J. Phisiol. Paris 73, 251-259.

[210] Szolcsányi, J. (2004): Forty years in capsaicin research for sensory pharmacology and physiology. Neuropeptide 38, 377-384.

[211] Tominata, M., Caterina, M.J., Malmberg, A.B., Rosen, T.A., Gilbet, H., Skinner, K., Raumann, B.E., Basbaum, A.I., Julius, D. (1998): The cloned capsaicin receptor integrates multiple pain-producing stimuli. Neuron 21:531-543.

[212] Taurog, A., Dorris, M., Doerge, D.R. (1994): Evidence for a radical mechanism in peroxidase-catalyzed coupling. I. Steady-state experiments with various peroxidases. Arch Biochem Biophys 315:82-87.

[213] Takeuchi, K. (2006): Unique profile of lafutidine: a novel histamine H2-receptor antagonist: mucosal protection thronghout GI mucosal mediated by capsaicin-sensitive afferent nerves, Acta Pharmacol. Sinica Suppl. 27-35

[214] Takeuchi, K., Ohuchi, T., Okabe, S. (1994): Capsaicin-sensitive sensory neurons in healing of gastric lesions induced by $\mathrm{HCl}$ in rats. Dig. Dis. Sci. 39: 2543-2546

[215] Tanne, H.J. (2006a): NEJM stands by its criticism of Vioxx. Brit. Med. J. 332:505.

[216] Tanne, H.J. (2006b): NEJM editor gives pretrial evidence in Vioxx case. Brit. Med. J. 332: 255.

[217] Tanne, H.J. (2006c): Court awards claim 13.3 M dollars in rofecoxib lawsuit. Brit. Med. J. 332:927.

[218] Todd, P.A., Clissold, S.P. (1990): Naproxen. A reappraisal of its pharmacology, and therapeutic use in rheumatic disease and pain states. Drugs 40: 91-137.

[219] Tramontana, M., Renzi, D., Calabro, A., Panerai, C., Milani, S., Surrenti, C., Evangelista, S. (1994): Influence of capsaicin-sensitive afferent fibres on acetic acid-induced chronic gastric ulcers in rats. Scand. J. Gastroenterol. 29: 406-413 
[220] Vaishnava, P., Wang, D.H. (2003): Capsaicin sensitive-sensory nerves and blood pressure regulation. Curr.Med. Chem. Cardiovasc. Hematol. Agents. 1: 177-188.

[221] Varga, L. (1936): Action of various stimulants on gastric chemistry (in Hungarian). Orv. Hetil. Hung. Med. Weekly J. 80: 702-704.

[222] Viranuvatti, V., Kalayasiri, C., Chearani, O. (1972): Effect of capsicum solution on human gastric mucosa as observed gastroscopically. Am. J. Gastroenterol. 58: 225-232.

[223] Walpole, C.S, Wrigglesworth, R., Bevan, S., et al. (1993b): Analogs of capsaicin with agonist activity as novel analgesic agents; structure-activity studies 1 . The aromatic "A-region". J. Med. Chem. 36, 2362-2372.

[224] Walpole, C.S, Wrigglesworth, R., Bevan, S., et al. (1993a): Analogs of capsaicin with agonist activity as novel analgesic agents; structure-activity studies 2 . The amide bond "B-region". J. Med. Chem. 36, 2373-2380.

[225] Walpole, CS, Wrigglesworth, R., Bevan, S., et al. (1993b): Analogs of capsaicin with agonist activity as novel analgesic agents; structure-activity studies 3 . The hydrophobic side chain "C-region". J. Med. Chem. 36, 2381-2389)

[226] Winter, J., (1987): Characterization of capsaicin sensitive neurons in adult rat dorsal root ganglion culture. Neurosci. Lett. 80: 134-140

[227] Wehmeyer, Y., Kasting, G.B., Powell, J.H., Kuhlenbeck, D.L., Underwood, R.A., Bowman, L.A. (1990): Applications of liquid chromatography with on-line radiochemical detection to metabolism studies on a novel class of analgesic. J. Pharm. Biomed. Anal. 8:177-183.

[228] Whittle, B.J.R. (1977): Mechanisms underlying gastric mucosal damage induced by indomethacin and bile salts, and the action of prostaglandins. Br. J. Pharmacol. 60: 455-460.

[229] Whittle, B.J.R., Lopez-Belmonte, J. (1991): Interactions between the vascular peptide endothelin-1 and sensory neuropeptides on gastric mucosal injury. Br. J. Pharmacol. 102: 950-954.

[230] Wood, J.N, Winter, J., James, I.F., Rang, H. Ph., Yeats, J., Bevan, S. (1988): Capsaicin induced ion fluxes in dorsal root ganglion neurons in culture. J. Neurosci. 8: $3208-3220$

[231] Yagi T (1990): Inhibition by capsaicin of NADH-quinone oxidoreductases is correlated with the presence of energy-coupling site 1 in various organisms. Arch. Biochem. Biophys. 281:305-311. 